\author{
Mon ograph \\ urn:1sid:zoobank.org:pub:F362301F-08C1-4660-8583-491BFEA32488
}

\title{
Revision of the spider genus Stygopholcus (Araneae, Pholcidae), endemic to the Balkan Peninsula
}

\author{
Bernhard A. HUBER ${ }^{1, *}$, Martina PAVLEK ${ }^{2}$ \& Marjan KOMNENOV ${ }^{3}$ \\ ${ }^{1}$ Zoological Research Museum Alexander Koenig, Bonn, Germany. \\ ${ }^{2}$ Croatian Biospeleological Society, Zagreb, Croatia. \\ ${ }^{2}$ Ruđer Bošković Institute, Zagreb, Croatia. \\ ${ }^{3}$ Blwd Kuzman Josifovski Pitu, 1000 Skopje, Macedonia. \\ *Corresponding author: b.huber@leibniz-zfmk.de \\ 2Email: martina.pavlek@gmail.com \\ ${ }^{3}$ Email: mkomnenov@gmail.com \\ $1 \odot$ https://orcid.org/0000-0002-7566-5424 \\ $2 \odot$ https://orcid.org/0000-0001-6710-0581 \\ 3 ○ https://orcid.org/0000-0001-6830-1043 \\ ${ }^{1}$ urn:1sid:zoobank.org:author:0000-0002-7566-5424 \\ ${ }^{2}$ urn:1sid:zoobank.org:author:0000-0001-6710-0581 \\ ${ }^{3}$ urn:1sid:zoobank.org:author:0000-0001-6830-1043
}

\begin{abstract}
The genus Stygopholcus Kratochvíl, 1932 is endemic to the Balkan Peninsula and includes only four nominal species: the epigean S. photophilus Senglet, 1971 in the south (Greece to Albania) and the 'northern clade' consisting of three troglophile species ranging from Croatia to Albania: S. absoloni (Kulczyński, 1914); S. skotophilus Kratochvíl, 1940; and S. montenegrinus Kratochvíl, 1940 (original rank re-established). We present redescriptions of all species, including extensive data on ultrastructure, linear morphometrics of large samples, and numerous new localities. We georeference previously published localities as far as possible, correct several published misidentifications, and clarify nomenclatorial problems regarding the authority of Stygopholcus and the identity of the type species S. absoloni. We suggest that the 'northern clade' has a relict distribution, resulting from past and present geologic and climatic factors. Future work on Stygopholcus should focus on the southern Dinarides, combining dense sampling with massive use of molecular data.
\end{abstract}

Keywords. Smeringopinae, Balkan Peninsula, cave spider, troglophile, relict.

Huber B.A., Pavlek M. \& Komnenov M. 2021. Revision of the spider genus Stygopholcus (Araneae, Pholcidae), endemic to the Balkan Peninsula. European Journal of Taxonomy 752: 1-60. https://doi.org/10.5852/ejt.2021.752.1391 


\section{Introduction}

Pholcid spiders have their highest species diversity in the tropics and subtropics, with $\sim 93 \%$ of all published records (excluding synanthropic species) between $40^{\circ} \mathrm{S}$ and $40^{\circ} \mathrm{N}$ (Huber \& Chao 2019; B.A. Huber, unpubl. data). Compared to many other spider families such as Linyphiidae Blackwall, 1859, Lycosidae Sundevall, 1833, and Gnaphosidae Banks, 1892 (Marusik \& Koponen 2002), Pholcidae C.L. Koch, 1850 do not seem to tolerate low temperatures, with few exceptions (e.g., Bennett 2014; Huber 2011a, 2014). As a result, continental Europe is poor with respect to pholcid spider diversity (Nentwig et al. 2020). Apart from several introduced synanthropic species and some widespread Mediterranean species of uncertain geographic origin [Holocnemus pluchei (Scopoli, 1763); Spermophora senoculata (Dugès, 1836)], the few autochthonous species are largely restricted to three areas: the western Mediterranean (Spermophorides Wunderlich, 1992 and Holocnemus Simon, 1873), eastern Europe (Pholcus Walckenaer, 1805), and the Balkan Peninsula (Stygopholcus Kratochvíl, 1932).

Among these, the Balkan Peninsula is the only European region with an endemic pholcid genus, Stygopholcus. This is noteworthy but unsurprising. The western part of the peninsula in particular is widely recognized as one of the regions in Europe with the highest species diversity (Griffiths et al. 2004), and for many taxa the level of endemism is the highest in Europe (e.g., Gottstein-Matočec et al. 2002; Eastwood 2004; Tzedakis 2004; Džukić \& Kalezić 2004; Sket et al. 2004; Mammola et al. 2019; Bregović et al. 2019; Lukić et al. 2020). Several factors have been proposed to account for this exceptional biological richness, including historical aspects (glacial refugia, land bridges) and present geography (terrain complexity, transition zone), geology (karst, caves), and climate variability (Mediterranean, mountainous, and continental) (Kryštufek \& Reed 2004; Reed et al. 2004; Džukić \& Kalezić 2004; Miheve et al. 2010).

Some spider genera share this exceptional species diversity on the Balkan Peninsula, as for example Troglohyphantes Joseph, 1882, Harpactea Bristowe, 1939, and Dysdera Latreille, 1804 (DeelemanReinhold 1978; Deeleman-Reinhold \& Deeleman 1988; Deltshev 2004; Komnenov 2009, 2020; Platania et al. 2020). By contrast, Stygopholcus is not particularly diverse. Three of the four species we recognize below have been known since Kratochvíl's (1940) diligent revision, and the fourth species was added half a century ago by Senglet (1971). Stygopholcus spiders are large and conspicuous; in the western Balkan they are among the first animals any biologist will encounter upon entering a cave. Thus, we have no reason to expect that a substantial number of species remain to be discovered. Nevertheless, we deemed this revision necessary for three main reasons.

First, species limits are not always obvious. For example, the similarity between the two species described by Kratochvíl (1940) prompted Senglet (1971) to reduce them to subspecies. Numerous misidentifications in the literature are further testimony to the difficulty in distinguishing some of the species, in particular females. Our revision is based on many more specimens from many more localities than available to previous authors, and we additionally support species limits with morphometric data. In addition, we point out deviating specimens from particular caves or areas that we tentatively assign to known species but that deserve further study.

Second, precise geographic data are a fundamental aspect of taxonomic work, but many previously published collecting sites are difficult to locate. We thus made a major effort to georeference published localities, using a simple scheme to point out uncertainties (see Material and methods).

Third, this revision is part of a series that aims to result in a full cladistic morphological analysis of Smeringopinae. This subfamily contains two sub-Saharan genera (Smeringopus Simon, 1890; Smeringopina Kraus, 1957; revised in Huber 2012, 2013), the mysterious Seychelles endemic Cenemus Saaristo, 2001, and a clade ranging from northern Africa to Central Asia (including Hoplopholcus 
Kulczyński, 1908, revised in Huber 2020; Crossopriza Simon, 1893; Holocnemus Simon, 1873; and Stygopholcus). To support a future comprehensive analysis of the entire subfamily, we did not limit our attention to characters useful for species delimitation but also focused on characters supposed to be useful for reconstructing the phylogeny of the subfamily (in particular SEM characters). We briefly discuss intergeneric relationships, but a more complete analysis will only be possible when the remaining two genera are revised (Crossopriza Simon, 1893 and Holocnemus Simon, 1873; revision in progress).

\section{Material and methods}

Taxonomic descriptions follow the style of recent publications on Smeringopinae (e.g., Huber 2020; based on Huber 2000). Measurements were done on a dissecting microscope with an ocular grid and are in $\mathrm{mm}$ unless stated otherwise; eye measurements are $\pm 5 \mu \mathrm{m}$. Photographs were made with a Nikon Coolpix 995 digital camera $(2048 \times 1536$ pixels $)$ mounted on a Nikon SMZ 18 stereo microscope or a Leitz Dialux 20 compound microscope. CombineZP (https://combinezp.software.informer.com/) was used for stacking photos. Drawings are partly based on photos that were traced on a light table and later improved under a dissecting microscope, or they were directly drawn with a Leitz Dialux 20 compound microscope using a drawing tube. Cleared epigyna were stained with chlorazol black. For SEM photos, specimens were dried in hexamethyldisilazane (HMDS) (Brown 1993), and photographed with a Zeiss Sigma 300 VP scanning electron microscope. SEM data are presented within the descriptions but are usually not based on the specific specimen described. The number of decimals in coordinates is supposed to give a rough indication about the accuracy of the locality data: four decimals means that the collecting site (or cave entrance) is within about $10 \mathrm{~m}$ of the indicated spot; three decimals: within $\sim 100 \mathrm{~m}$; two decimals: within $\sim 1 \mathrm{~km}$; one decimal: within $\sim 10 \mathrm{~km}$. Coordinates for some caves were taken from the GeoRef portal (http://www.georef.hr/) and Bioportal - Katastar speleoloških objekata RH (http://www.bioportal.hr/gis/), hosted by the Institute for Environment and Nature of Croatia. Distribution maps were generated with ArcMap ver. 10.0.

Original cave names are used as much as possible, i.e., the Serbo-Croatian pećina, the Croatian špilja, and the Greek spilaio are mostly used instead of 'Cave'; the Serbo-Croatian jama means pit, the SerboCroatian ponor means sinkhole.

\section{Institutional abbreviations}

This study is based on the examination of more than 800 adult specimens from approximately 200 localities, deposited in the following collections:

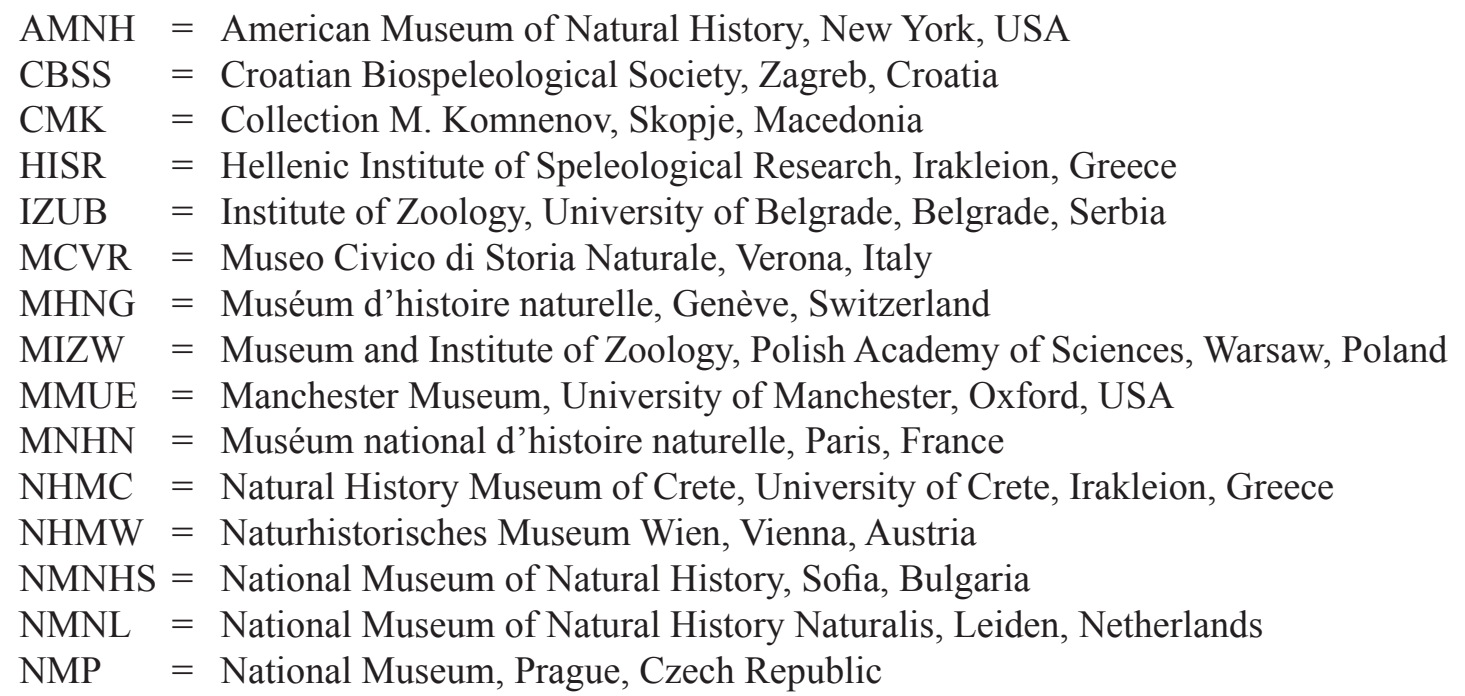


ROC = Collection Roman Ozimec, Zagreb, Croatia

SMF = Forschungsinstitut und Naturmuseum Senckenberg, Frankfurt, Germany

ZCSL = Zoological collection of SubBio Lab, University of Ljubljana, Ljubljana, Slovenia

ZFMK = Zoologisches Forschungsmuseum Alexander Koenig, Bonn, Germany

$\mathrm{ZMB}=$ Museum für Naturkunde, Berlin, Germany

\title{
Morphological abbreviations used in the text
}

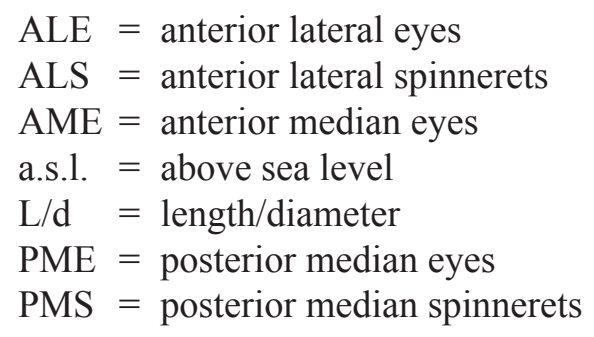

Abbreviations used in figures only are explained in the figure legends.

\section{Results}

\author{
Class Arachnida Lamarck, 1801 \\ Order Araneae Clerck, 1757 \\ Family Pholcidae C.L. Koch, 1850 \\ Genus Stygopholcus Kratochvíl, 1932
}

Stygopholcus Kratochvíl, 1932: 2-3 (type species: Holocnemus absoloni Kulczyński, 1914; see below).

Stygopholcus - Absolon \& Kratochvíl 1932: 75. — Kratochvíl 1934: 182; 1940: 8; 1978: 27 — Senglet 1971: 354; 2001: 58, 65. — Brignoli 1971: 257; 1976: 561.

\section{Authority of Stygopholcus}

There has long been disagreement about the publication that has made the genus name Stygopholcus validly available. Some cataloguers such as Roewer (1942) and Brignoli (1983) considered the original description to be in Kratochvíl (1940), while Bonnet (1958) cited Absolon \& Kratochvíl (1932) as the source for the name. Platnick (1993) first opted for Kratochvíl (1940), later (Platnick 2000) for Absolon \& Kratochvíl (1932). The World Spider Catalog (2020) adopted Platnick's later view. A third option, namely Kratochvíl (1932), was offered by Kratochvíl himself (Kratochvíl 1940), and adopted by Senglet (1971).

The name Stygopholcus is first mentioned in two papers in 1932: in one of these, Absolon \& Kratochvíl (1932) explicitly refer to the other publication (Kratochvíl 1932) as the source of the name:

"Mit dem Namen Stygopholcus n. g. hat J. Kratochvíl (siehe "Sur quelques Araignées de Slavonie central", Bulletin de l'Institut national agronomique, Sign. C. 23, Brno 1932) die Art Holocnemus (Hoplopholcus) absoloni Kulcz. als selbständige Gattung abgetrennt." ["With the name Stygopholcus n. g. , J. Kratochvil (see "Sur quelques Araignées de Slavonie central”, Bulletin de l'Institut national agronomique, Sign. C. 23, Brno 1932) has moved the species Holocnemus (Hoplopholcus) absoloni Kulcz. to constitute a separate genus"].

Other than this statement, Absolon \& Kratochvíl (1932) just say a few words about the habitat of S. absoloni. Absolon \& Kratochvíl (1932) thus fail to satisfy a requirement of ICZN (1999) Article 
13.1.1 for names published after 1930 to be available: “... be accompanied by a description or definition that states in words characters that are purported to differentiate the taxon." The fact that Absolon \& Kratochvíl (1932) contains a bibliographic reference to such a published statement could be construed as satisfying ICZN Article 13.1.2. We do not know which 1932 paper was published first. However, if Kratochvíl (1932) was published first, it has priority for that reason; if it was published second, it has priority because Absolon \& Kratochvíl (1932) refer to a statement that was not yet published at the time.

Confusingly, the paper to which Absolon \& Kratochvíl (1932) refer was originally also not intended to be the source of the name. Kratochvíl (1932) referred to "Stygopholcus n. g. in lit., jejž popíši na jiném mistě" ["Stygopholcus n.g. in lit., that I will describe elsewhere"], suggesting that the genus name was intended to be made available in an upcoming publication (possibly Kratochvíl 1934). However, Kratochvíl (1932) provides "characters that are purported to differentiate the taxon", satisfying ICZN Article 13.1.1; and he explicitly names " $H$. absoloni Kulcz." as the only species to be included in the genus, satisfying the requirements of ICZN Article 13.3 regarding the fixation of a type species. From a formal perspective, Kratochvíl's (1932) intentions (of making the name available later) are as irrelevant as the confusion between genus rank (in the Czech text) and subgenus rank (in the French summary), and the minimalist nature of his diagnosis: he mentions that (1) the female sternum process present in Holocnemus pluchei is absent in Stygopholcus; (2) the abdomen shape differs between Holocnemus and Stygopholcus; (3) the female palp of Stygopholcus resembles that of Pholcus rather than that of Holocnemus pluchei (which is enlarged); and (4) Stygopholcus has cheliceral stridulation like Holocnemus (in contrast to Pholcus).

We conclude that Stygopholcus was made available by Kratochvíl (1932).

\section{Identity of Stygopholcus absoloni (Kulczyński, 1914)}

In the original description of "Holocnemus (Hoplopholcus) Absolonii", Kulczyński (1914) mentions two caves near Trebinje by name: "Mares et feminae lecta sunt prope Trebinje in speluncis: Ilijina pećina ... et Zovica jama..." ["males and females were collected in caves near Trebinje: Ilijina cave ... and Zovica pit ..."]. He then notes that according to Absolon ("Teste Cel. C. Absolon"), this species has been found in many other caves in southeastern Herzegovina (southern region of modern Bosnia and Herzegovina). This strongly suggests that he had only seen the specimens from the two caves near Trebinje. Any specimens he had from these caves, and only these specimens, constitute the type series (syntypes).

In Kulczyński's (1914) long original text, the morphology is described in considerable detail but complex shapes are notoriously difficult to understand from text alone. However, he was obviously aware of differences among the specimens available to him, starting a paragraph with "Maris palpi variant paululo formâ, etiam mandibularum armatura paulo mutabilis" ["the male palps vary slightly in shape, and also the armature of the chelicerae is slightly variable"]. He clearly interpreted this as intraspecific variation, but without specifying to which variant(s) his four figures referred.

When Kratochvíl (1940) made a first careful and detailed revision of Stygopholcus, he realized that Kulczyński's (1914) figures represented the two variants, which Kratochvíl interpreted (correctly) as different species. He considered Kulczyński's (1914) fig. 25 to represent one species, figs 24, and 2627 the other species. This view was accepted by subsequent cataloguers and authors, also by Senglet $(1971,2001)$, the only author other than Kratochvíl who studied Stygopholcus in some detail. Since Kulczyński's (1914) figures of the male palps are in different views, we checked Kratochvíl's (1940) distinction of the two putative species by orienting palps of both species in exactly the same positions as in Kulczyński's (1914) figures. There is no doubt that Kratochvíl (1940) was right in this respect. 
Kratochvíl's (1940) error was that he felt either entitled or obliged (or both) to designate new types from a new type locality for $S$. absoloni, choosing a cave $\sim 35 \mathrm{~km} \mathrm{NW}$ of Trebinje, "Grabova peć kod Grabova dola". We can only speculate about his motivation, but it seems reasonable to assume that he had been postponing his detailed revision of the genus for many years (at least since 1932), hoping to eventually be able to see Kulczyński's type series. This hope was frustrated, as indicated in a footnote (Kratochvíl 1940: 16) where he explicitly stated that the specimens from Ilijina pećina and Zovica jama were not accessible to him. Unfortunately, among his rich new material there were no specimens from any of these two caves.

We made a major effort to locate Kulczyński's types, but found only one vial, deposited in the Museum \& Institute of Zoology, PAS, in Warsaw, Poland. The supposed types of S. absoloni deposited in Prague and listed in Růžička et al. (2005) are Kratochvíl's erroneous 'types' from “Grabova peć kod Grabova dola”. Kulczyński's true type vial contains a label in his own handwriting, but the only locality information on this label is "Trebinje". A second, very detailed label says "Ilijina pećina", but other information on this label leaves no doubt that it was added much later. Fortunately, Kulczyński's original catalogue (in Warsaw) offers a clear hint about the origin of the specimens in the vial. It says "Jaskinie koło Trebinje" ["caves near Trebinje"], suggesting that Kulczyński joined the specimens from Ilijina pećina and Zovica jama.

This type vial contains only three specimens, one male and two females. This is clearly only part of Kulczyński's material, because he obviously had at least two males available. However, both variants/ species are represented in this vial. Circumstantial evidence suggests that one female is from Ilijina pećina (it corresponds to more recently collected females from this cave). This forces us to conclude that the other specimens (one male and one female) are from Zovica jama.

This is clearly a case where a lectotype should be designated for $S$. absoloni in order to provide stability and avoid further confusion about the basics (ICZN Recommendation 74G). However, it is not obvious which specimen would best serve as a lectotype. Selecting the male specimen has one major disadvantage: it makes Zovica jama the type locality, a cave that we were not able to locate, and that was even unknown to local cavers in Trebinje we met in 2014. On the other hand, Ilijina pećina is well known and easily accessible, providing a precise type locality. Selecting the female from Ilijina pećina would thus seem to better satisfy ICZN Recommendation 74E (Verification of locality).

At the same time, selecting the male specimen as lectotype has also advantages. Most importantly, it preserves the prevailing usage of the name. This male is the same variant/species as Kratochvíl's (1940) S. absoloni 'types' from "Grabova peć kod Grabova dola", and it also corresponds to what Senglet (1971) interpreted as S. absoloni. Thus, selecting the male satisfies ICZN Recommendation 74A (Agreement with previous restriction). A second (minor) advantage is the fact that species in Stygopholcus are slightly easier to distinguish by males than by females (as in most Pholcidae).

Thus, we decided to select the male as lectotype, mainly for two reasons: it seems to better serve stability, one of the major guiding principles of the Code. Second, it is possible that the exact location of Zovica jama will eventually be determined, weakening the argument against selecting the male.

\section{Diagnosis}

Stygopholcus includes relatively large (total body length: 5-6), long-legged spiders with oval abdomen (Figs 1-5). Males of Stygopholcus are easily distinguished from all other Smeringopinae by modified (club-shaped) hairs frontally on chelicerae (Figs 19, 51); also by unique transparent or weakly sclerotized dorsal bulbal process (arrows in Figs 17, 117) and by distinct brushes of hair-shaped retrolateral 
membranous processes at tip of procursus (Figs 29, 67, 99, 137). Males and females differ from other Smeringopinae by tarsal organs of legs with undulating rim (Figs 38, 75, 97, 142).
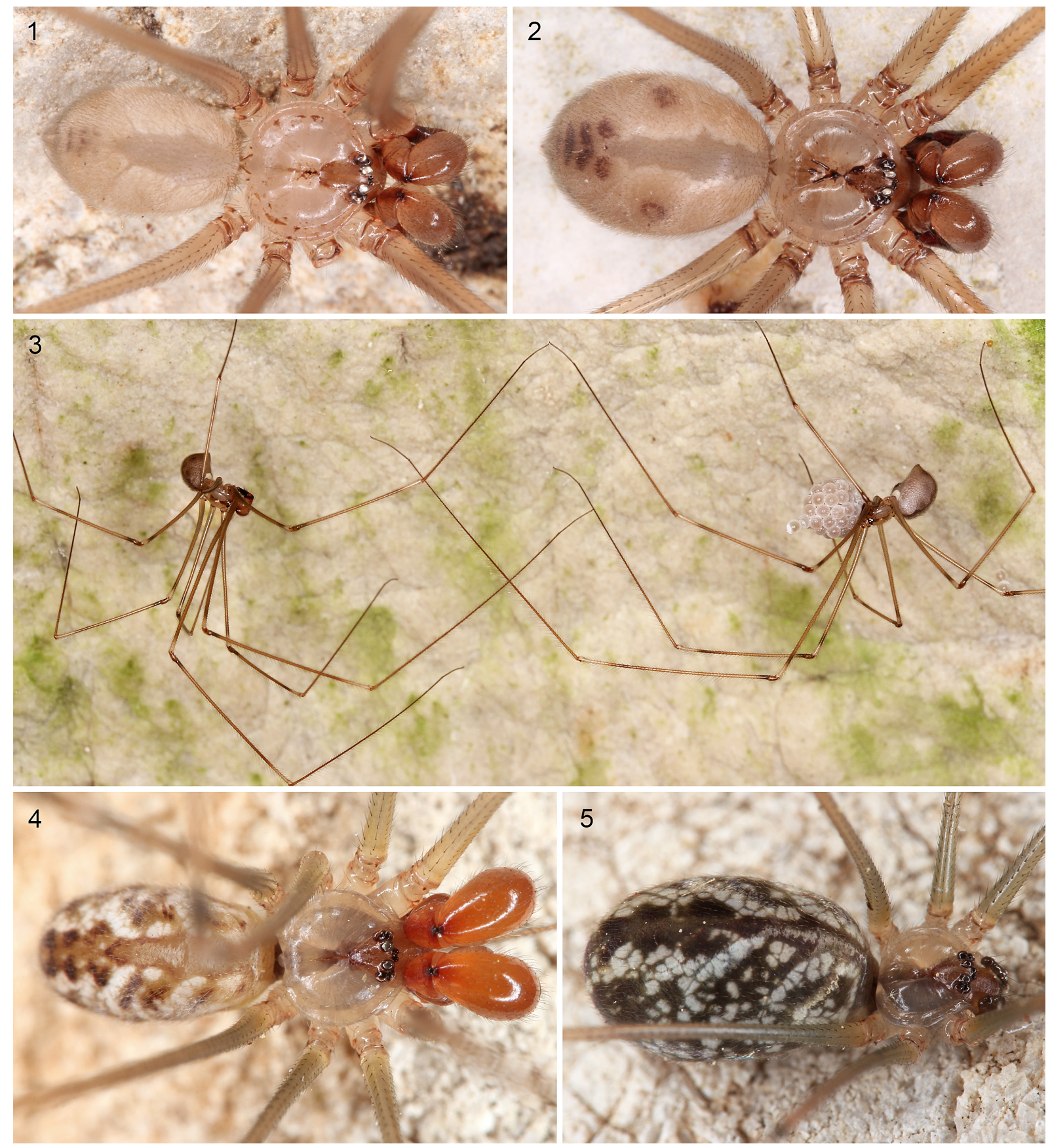

Figs 1-5. Stygopholcus Kratochvíl, 1932; live specimens. 1. S. absoloni (Kulczyński, 1914); male from Vjetrenica, Bosnia and Herzegovina. 2. S. skotophilus Kratochvíl, 1940; male from Pavlova pećina, Bosnia and Herzegovina. 3. S. montenegrinus Kratochvíl, 1940; male and female with egg-sac from Studenačka pećina, Montenegro. 4-5. S. photophilus Senglet, 1971; male and female from Episkopi, Crete. Photos: BAH. 


\section{Description}

Male

Body. Total body length $\sim 5-6$; carapace width $\sim 1.5-2.5$. Carapace with deep central pit and pair of shallow furrows diverging from posterior side of pit toward posterior rim (Figs 39, 61, 91, 107); ocular area slightly raised, eye triads relatively close together (distance PME-PME similar to PME diameter), each secondary eye (especially PME) accompanied by indistinct elevation (arrows in Fig. 61; "pseudoeyes"; cf. Huber 2009), AME relatively large (usually 40-50\% of PME small diameter). Clypeus high, unmodified or with indistinct modified area medially (arrow in Fig. 129). Abdomen oval, never elevated or pointed above spinnerets (Figs 1-5). Male gonopore with 4-8 epiandrous spigots (Figs 62, 92, 143), ALS with only two spigots each: one large widened spigot and one pointed spigot (Figs 69, 135); PMS with two spigots each (cf. female, Fig. 42); PLS without spigots.

Color. In general ochre-yellow to brown, with distinct dark pattern in S. photophilus, without or with reduced dark pattern in other (troglophile) species. Carapace mostly pale, with darker ocular area and posterior triangle (Figs 1-2, 4); sternum either dark brown to black (S. photophilus), or light brown, with darker brown radial marks (other species). Legs in S. photophilus with dark rings on femora (subdistally) and tibiae (proximally and subdistally) and with short dark longitudinal lines dorsally on femora (Figs 127-128); in other species monochromous, without dark rings and longitudinal lines. Abdomen in S. photophilus with distinct dorsal and ventral patterns; in other species with fewer dorsal and lateral dark marks restricted to posterior part and without or with indistinct ventral pattern.

Chelicerae. Chelicerae with 1-4 modified (cone-shaped to club-shaped) hairs on each distal cheliceral apophysis and $\sim 10-40$ club-shaped hairs on frontal face (Figs 19, 27-28, 51, 63-64, 93-94, 119, 130, 132); in S. photophilus with strongly sculptured median edge (Fig. 131); with stridulatory ridges (Figs 35, 71, 95, 133), distances between ridges $\sim 6-9 \mu \mathrm{m}$ in 'northern clade', $\sim 3 \mu \mathrm{m}$ in S. photophilus.

PALPS. In general as in Figs 6-11; coxa with rounded retrolateral hump; trochanter barely modified; femur widening distally, slightly curved towards dorsal, with proximal retrolateral process, indistinct transversal dark line on retrolateral side, and stridulatory pick (modified hair) on prolateral side (Figs 65, 103); femur-patella joints shifted toward prolateral side (arrows in Fig. 6); tibia-tarsus joints shifted toward retrolateral side (arrows in Fig. 8); palpal tarsus without dorsal macrotrichia, palpal tarsal organ exposed (Figs 30, 104); procursus dorsally with approximately five (S. photophilus) to ten (other species) weakly to strongly curved hairs; procursus without ventral 'knee', distally with strong ventral spine, with dorsal and prolateral processes of variable size, and distinctive brushes of hair-shaped retrolateral membranous processes (Figs 29, 67, 99, 137); genital bulb with basal sclerite connecting to tarsus (bs in Figs 15, 118), with small and weakly sclerotized (S. photophilus) or transparent (other species) dorsal process (arrows in Figs 17, 117), and main sclerite consisting of retrolateral and dorsal processes (rp and dp in Figs 15-18, 118); sperm duct opening directly on genital bulb at basis of dorsal process (arrow in Fig. 32).

Legs. Legs long and relatively thin, leg 1 length $\sim 35-60$, tibia 1 length $\sim 10-15$, tibia 2 longer than tibia $4(1.1-1.2 \times)$. Tibia $1 \mathrm{~L} / \mathrm{d} \sim 60-75$. Femur 1 usually with spines ventrally in one row (S. photophilus) or two rows (other species) (Fig. 73), in very small males rarely without spines; spines proximally gradually transforming into regular setae; spines never present on femur 2 or on tibia 1; legs without curved hairs; with few short vertical hairs; retrolateral trichobothrium in proximal position (at $2-4 \%$ of tibia length in tibia 1), prolateral trichobothrium absent on tibia 1, present on other tibiae. Tarsal pseudosegments very indistinct, never regular rings but rather indistinct irregular platelets. Tarsus 4 with two rows of prolatero-ventral comb-hairs (Figs 34, 74; see also Huber \& Fleckenstein 2008: fig. 13). Tarsal organs of legs capsulate, with weakly (S. photophilus) to strongly (other species) undulating rim (Figs 75, 97). 


\section{Female}

In general very similar to male; chelicerae with less distinct or without stridulatory files (Figs 36, 72, 96, 134); legs slightly shorter than in male, without spines. All tarsal organs capsulate (i.e., also on palps; Figs 37, 109, 141). Representatives of the 'northern clade' with pair of indistinct processes posteriorly
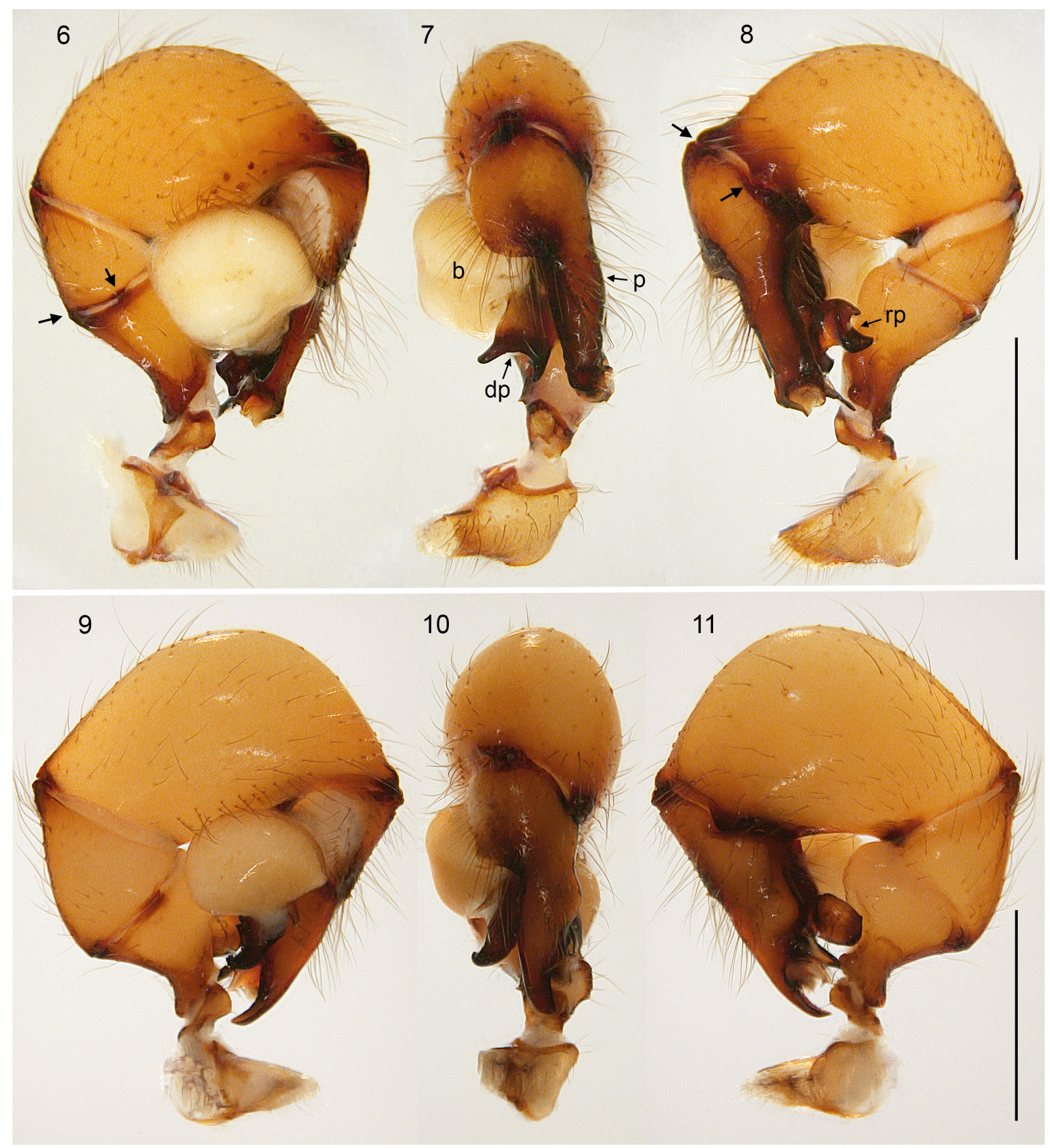

Figs 6-11. Stygopholcus Kratochvíl, 1932; left male pedipalps, prolateral, dorsal, and retrolateral views. 6-8. S. skotophilus Kratochvíl, 1940; from Ilijina pećina, Bosnia and Herzegovina (ZFMK (Ar 22198)). 9-11. S. photophilus Senglet, 1971; from Episkopi, Crete (ZFMK (Ar 22205)). Abbreviations: $b=$ genital bulb; $\mathrm{dp}=$ dorsal process (of genital bulb); $\mathrm{p}=$ procursus; $\mathrm{rp}=$ retrolateral process (of genital bulb). Arrows point at joints shifted toward prolateral (femur-patella) and retrolateral (tibia-tarsus). Scale bars: $1 \mathrm{~mm}$. 
on carapace (arrows in Figs 39,107) acting against pair of poorly visible plates on abdomen. Epigynum usually consisting of large, simple anterior plate and short but wide posterior plate (Figs 23, 55, 85, 123); in S. photophilus with pair of bulging areas in front of anterior plate (arrows in Fig. 144). Internal genitalia (Figs 145-156) with sclerotized arc that consists of dorsal and ventral parts (da and va in Figs 153,156$)$ and is variably visible in uncleared specimens; uterus externus with median ventral pouch, usually clearly visible as round or oval structure in untreated specimens (e.g., Figs 55-60); with pair of sclerites originating from posterior margin and either slightly converging anteriorly or parallel (arrows in Figs 25, 125), indistinct in S. skotophilus; pore plates large, flat, with homogeneously distributed pores (Figs 22, 54, 84, 122).

\section{Relationships}

Stygopholcus is morphologically very similar and geographically very close to Hoplopholcus (Huber 2020). This led to a debate between A. Senglet and P. Brignoli in the 1970s and 80s, on whether the two genera should be synonymized or not (Senglet 1971; Brignoli 1971, 1976, 1983). In the end, Senglet (2001) presented a series of morphological differences, and since version 2.5 of the World Spider Catalog (Platnick 2002) Stygopholcus has been listed as a valid genus.

The most recent molecular phylogeny of Pholcidae (Eberle et al. 2018) has suggested that Stygopholcus and Hoplopholcus are not even sister taxa, placing Stygopholcus closer to Crossopriza than to Hoplopholcus (with a 'reasonable' bootstrap support of 87). Morphological characters that support a sister-group relationship between Stygopholcus and Crossopriza are the female stridulatory apparatus between prosoma and abdomen and the dark lines on the legs. However, a convincing morphological analysis will require a detailed study of Crossopriza (a revision is in preparation) and of Holocnemus. The type species of Holocnemus (H. pluchei) was not included in the molecular analysis of Eberle et al. (2018), and the two species of Holocnemus that were included (H. caudatus and H. hispanicus) may in fact be misplaced in Holocnemus and belong to Crossopriza (B.A. Huber, unpubl. data).

The monophyly of Stygopholcus is strongly supported by molecular data (maximum bootstrap support; Eberle et al. 2018) and by several putative synapomorphies: (1) male chelicerae with club-shaped hairs on frontal face (Figs 19, 51); (2) unique undulating rim of leg tarsal organs (Figs 38, 75, 97, 142); (3) transparent to weakly sclerotized process dorsally on genital bulb (arrows in Figs 17, 117).

Within Stygopholcus, there is strong evidence that S. photophilus is sister to all other species (the 'northern clade'). Both a six genes approach (Eberle et al. 2018) and a study using hundreds of universal single-copy orthologs (L. Dietz et al., unpublished data) consistently recovered this relationship, and numerous morphological characters are shared by all species except $S$. photophilus. Some of these are likely to be synapomorphies: (1) spines on male femur 1 arranged in two rows rather than in just one (Fig. 73); (2) dorsal bulbal process with two separate tips (Figs 17-18, 49-50, 81-82) ; (3) troglophile mode of life and corresponding pale coloration (Figs 1-2).

Within the 'northern clade', there is equally strong support for the relationship absoloni + (skotophilus + montenegrinus). Several morphological characters shared by S. skotophilus and S. montenegrinus support this view, even though it is not clear yet if all of these shared similarities are synapomorphies: (1) procursus straight (rather than weakly curved toward femur as in S. absoloni) (Fig. 44); (2) distance between tips of dorsal bulbal process (bulbal measure D; Fig. 17) $>0.24(<0.24$ in S. absoloni) (Fig. 159); (3) size of bulb (bulbal measure E; Fig. 15) $>0.80(<0.78$ in S. absoloni) (Fig. 160).

\section{Natural history}

Stygopholcus photophilus can be classified as an epigean species. It tolerates direct sunlight, is often found in exposed webs among the vegetation, and enters caves only occasionally. Compared to epigean 
relatives in the genera Holocnemus, Hoplopholcus, and Crossopriza, it does not show any obvious troglomorphism. By contrast, all other congeners are slightly troglomorphic. While eye size and leg length do not seem to indicate troglomorphism, their general coloration is paler and they have fewer dark marks on the abdomen and legs. This reflects their strong preference for dark sheltered spaces like caves, deep fissures and cracks, and spaces under rocks in forests. Most records of these species are from caves, but this is probably due to the fact that access to caves is usually much easier for collectors than access to other shallow subterranean habitats.

According to Kratochvíl (1940), cave-dwelling Stygopholcus prefer dry caves, or dry areas of caves that include humid sections. We do not have quantitative data on this, but our impression was that Stygopholcus requires high humidity; specimens were occasionally found even on dripping wet rocks. They seem to prefer the twilight area, avoiding both direct sunlight at the cave entrance and complete darkness. As already noted by Kratochvíl (1940), individual specimens are occasionally found in deeper parts of caves. As usual in Pholcidae in general, they avoid caves or cave sections with strong air flow (Kratochvíl 1940).

Beyond basic habitat information, little is known about the biology of Stygopholcus spiders. Adult specimens of representatives of the northern clade were found throughout the year (20 of 186 collecting events between December and February); we have no records of S. photophilus from January and February. Our limited observations (MK, unpublished data) suggest that representatives of the northern clade produce egg-sacs between May and August. In a cave near Podgorica in Montenegro, the last author observed a mating of $S$. montenegrinus where the female kept holding on to her egg-sac while mating. Senglet (2001) observed mating in S. photophilus and studied genital mechanics by freezefixing mating pairs. He described rhythmic palpal movements during copulation, sperm uptake with a single thread held between legs 3 , and was able to ascribe specific functions to individual genitalic structures.

\section{Composition}

The genus includes four named species and this number is unlikely to change substantially in the future. However, some specimens assigned tentatively to S. absoloni, S. skotophilus, and S. montenegrinus, respectively, may represent separate species. A convincing resolution of species limits will probably require both a denser sampling and a massive use of molecular data.

\section{Distribution}

The genus is restricted to the Mediterranean part of the Balkan Peninsula, ranging from southern Croatia to Crete (Fig. 167). The epigean S. photophilus has a wide distribution, ranging from southern Albania to Crete. The three troglophile species of the 'northern clade', S. absoloni, S. skotophilus, and S. montenegrinus, range from southern Croatia to northern Albania (Fig. 168). They seem to be relicts that have survived further north by adapting to subterranean conditions (see Discussion).

\section{Identification key}

1. Distinct dark marks on entire abdomen, also dorsally in anterior half (Figs 4-5); leg femora and tibiae with dark rings; procursus distally with strongly projecting dorsal sclerite (ds in Figs 113, 137, 138); spines on male femur 1 in only one row; tibia $1>1.40 \times$ longer than tibia 2 in males (Fig. 165), $>1.45 \times$ in females (Fig. 166); strong transversal ridges on anterior part of epigynum (Figs 121, 144); epigynum width/length usually $<1.75$ (Fig. 163)

- Dark marks on abdomen dorsally restricted to posterior half (Figs 1-2); leg femora and tibiae without dark rings; dorsal sclerite distally on procursus distinct but not projecting (ds in Figs 13, 44); spines on male femur 1 in two rows; tibia $1<1.40 \times$ longer than tibia 2 in males (Fig. 165), $<1.45 \times$ in 
females (Fig. 166); epigynum with weak transversal ridges (e.g., Figs 23, 55); epigynum width/ length usually $>1.75$ (Fig. 163) 'northern clade' - 2

2. Prolateral sclerite distally on procursus large, heavily sclerotized, and triangular (arrow in Fig. 14), procursus slightly curved towards femur (Fig. 13), procursus short ( $<1.3$; Fig. 161), bulb measure D small $(<0.23$; Fig. 159); posterior sclerites in female internal genitalia parallel and close together (distance $\sim 0.2-0.4$; Figs 23-26, 164)

S. absoloni (Kulczyński, 1914)

- Prolateral sclerite distally on procursus small, weakly sclerotized, and cylindrical (arrows in Figs 45-46, 77-78), procursus straight (Fig. 44), procursus longer (usually $>1.3$, except in smallest specimens; Fig. 161), bulb measure D large (>0.24; Fig. 159); posterior sclerites in female internal genitalia either wider apart (Figs 55-60) or converging anteriorly (Figs 85-90)

3. Prolateral sclerite on procursus wide (Figs 45-46); bulb measure $C$ small $(<0.16$; Fig. 158); epigynal measure F large (usually $>0.39$, smaller in specimens from near Sedlari; Figs 55-60, 164)

S. skotophilus Kratochvíl, 1940

- Prolateral sclerite on procursus narrow (Figs 77-78); bulb measure C large (usually $>0.23$, smaller in eastern specimens; Fig. 158); epigynal measure F small (usually $<0.41$, larger in eastern specimens; Figs 164) S. montenegrinus Kratochvíl, 1940

Stygopholcus absoloni (Kulczyński, 1914)

Figs 1, 12-42, 145-146, 153

Holocnemus (Hoplopholcus) Absolonii Kulczyński, 1914: 355 (part), pl. 16, figs 24, 26-27 (not fig. 25).

Holocnemus (Stygopholcus) absoloni - Kratochvíl 1934: 181 (part; see Remarks below).

Hoplopholcus absoloni - Brignoli 1971: 257, fig. 20.

Stygopholcus absoloni - Kratochvíl 1932: 2; 1940: 10, figs 3, 4a-b, 5a-b, 7 (left); 1978: 28. — Absolon \&

Kratochvíl 1932: 75. - Senglet 1971: figs 37-39; 2001: 65, fig. 21.

"S110 Stygopholcus Bal3" - Eberle et al. 2018. — Huber et al. 2018.

\section{Misidentifications}

Holocnemus (Stygopholcus) absoloni - Kratochvíl 1934: 181 (part; see Remarks below); 1935: 11 (see Remarks below).

\section{Remarks}

For most of the localities listed in Kratochvíl (1934), the presence of $S$. absoloni could either be confirmed or appears very probable judging from the distribution of confirmed specimens. Exceptions are the following: Ilijina pećina and "Pećina Napode" (= Vilina pećina) (see S. skotophilus), Medja peć na Ilijina brdu, Vodena peć kod Sedlara, Grabova peć kod Sedlara, and Trovrata peć (tentatively assigned to S. skotophilus), and Jamutina [pećina] (see S. montenegrinus). Kratochvíl's (1934) "Pec kod Ljubova" [Ljubovo?] is not mentioned in the extensive list of localities in Kratochvíl (1940) and is thus not further considered herein.

By contrast, all caves listed in Kratochvíl (1935) probably contain S. skotophilus; this is either confirmed by new material or very likely judging from the distribution of confirmed specimens.

\section{Diagnosis}

Distinguished from other species in the 'northern clade' (S. skotophilus; S. montenegrinus) by large prolateral sclerite on procursus (distally narrowing; arrow in Fig. 14), by procursus slightly curved 


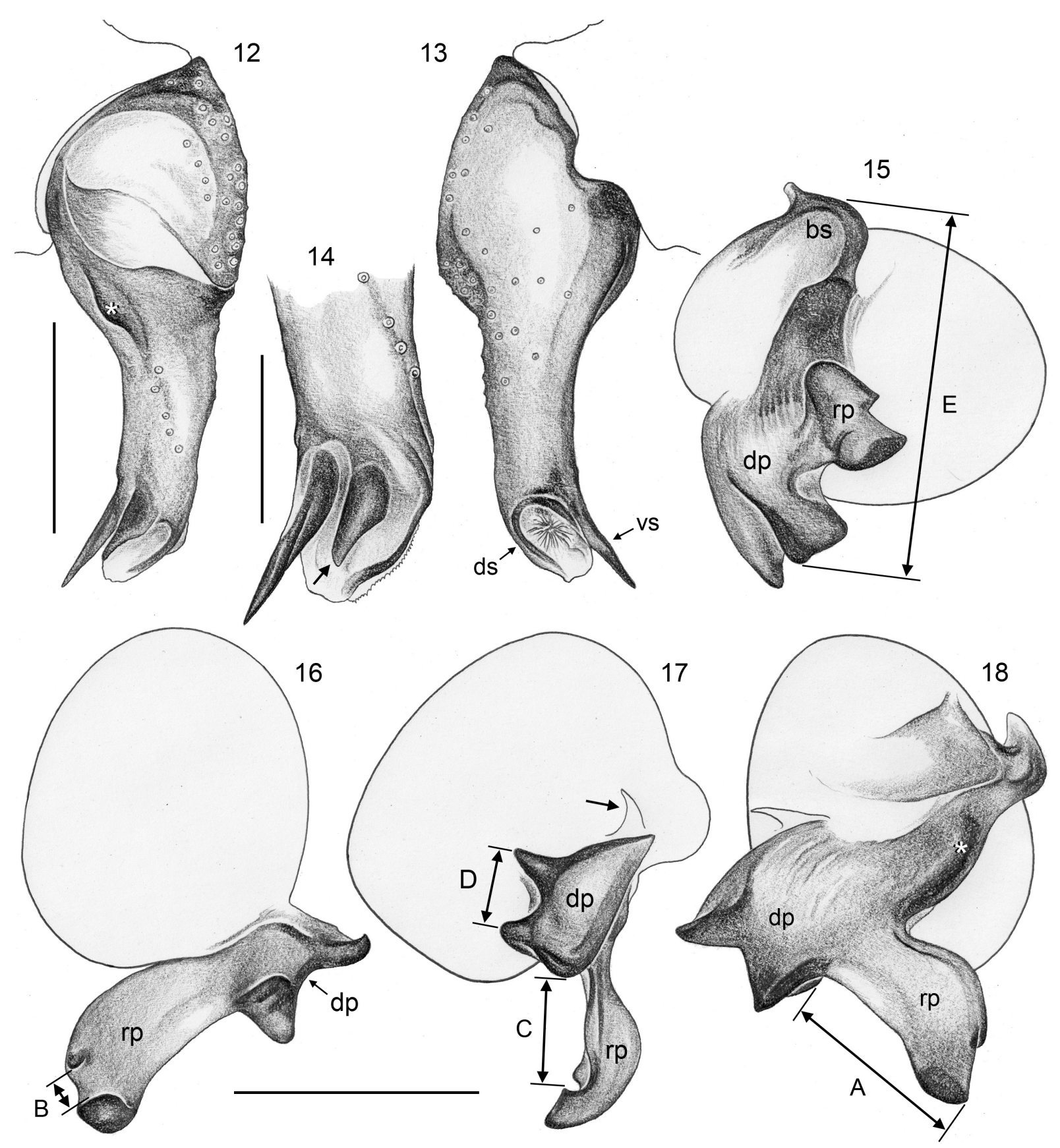

Figs 12-18. Stygopholcus absoloni (Kulczyński, 1914); male from Balićeva špilja, Croatia (ZFMK (Ar 22195)). 12-13. Left tarsus and procursus, prolateral and retrolateral views; asterisk in Fig. 12 marks prolateral hump. 14. Tip of left procursus, prolateral (slightly ventral) view; arrow points at distinctive prolateral sclerite. 15-18. Left genital bulb, retrolateral, ventral, prolateral-distal, and dorso-distal views, showing bulb measures A-E; arrow in Fig. 17 points at transparent bulbal process; asterisk in Fig. 18 marks connecting sclerite that is absent in specimen from Jama na Brezdanu. Abbreviations: $\mathrm{bs}=$ basal sclerite; $\mathrm{dp}=$ dorsal process; $\mathrm{ds}=$ dorsal sclerite; $\mathrm{rp}=$ retrolateral process; $\mathrm{vs}=$ ventral sclerite. Scale bars: $12-13,15-18=0.5 \mathrm{~mm} ; 14=0.25 \mathrm{~mm}$. 
toward femur (rather than straight; Fig. 13), by shorter procursus (Fig. 161; with little overlap), by shape of retrolateral bulbal process in retrolateral view (Fig. 15; small notch on ventral side), and by bulb measures: measure B smaller (Fig. 157; with little overlap), measure D smaller (Fig. 159; no overlap), and measure E smaller (Fig. 160; no overlap). From S. skotophilus also by more hairs on male cheliceral base (Fig. 162; with little overlap) and by larger bulb measure C (Fig. 158; no overlap), and by smaller epigynal measure F (Fig. 164; little overlap).

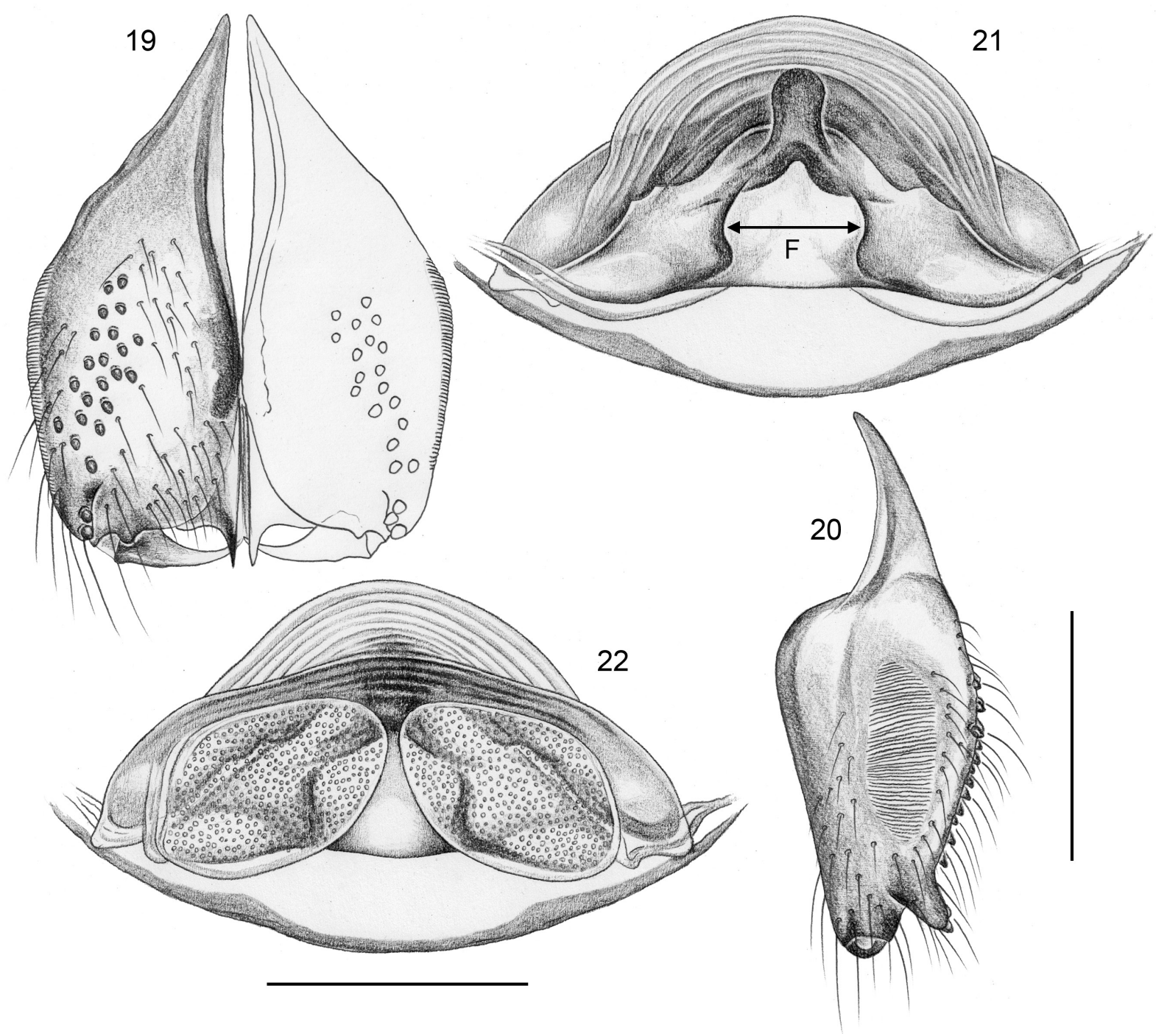

Figs 19-22. Stygopholcus absoloni (Kulczyński, 1914); male from Balićeva špilja, Croatia (ZFMK (Ar 22195)), female from Zavala, Bosnia and Herzegovina (MHNG). 19-20. Male chelicerae, frontal and lateral views. 21-22. Cleared female genitalia, ventral and dorsal views, showing epigynal measure F. Scale bars: $0.5 \mathrm{~mm}$. 


\section{Type material}

\section{Lectotype}

BOSNIA AND HERZEGOVINA • $\widehat{\jmath}$ (designated herein, examined); "near Trebinje, Zovica jama"; $\sim 42.7^{\circ} \mathrm{N}, 18.3^{\circ} \mathrm{E}$; date unknown (1912?); K. von Arenstorff leg.; MIZW.

\section{Paralectotype}

BOSNIA AND HERZEGOVINA • + , examined; same collection data as for lectotype; MIZW,

\section{Other material examined}

BOSNIA AND HERZEGOVINA • $1{ }^{\jmath}, 2$ 2 우 ; Vjetrenica pećina; $42.8459^{\circ} \mathrm{N}, 17.9837^{\circ} \mathrm{E} ; 280 \mathrm{~m}$ a.s.1.; 27 May 2014; B.A. Huber and M. Komnenov leg.; in cave near entrance and outside cave, hole in ground; ZFMK (Ar 22192) • 1 q, 1 juv., in pure ethanol; same collection data as for preceding; ZFMK (Bal12) • 1 ก, 1 O; Popovo polje, Zavala, Bjelušica, in cave; $42.8447^{\circ} \mathrm{N}, 17.9786^{\circ} \mathrm{E} ; 350 \mathrm{~m}$ a.s.1.; 2 Dec. 2014, M. Pavlek leg.; CBSS (Ar 3480) • 1 đ; same locality as for preceding; 26 Oct. 2015; M. Pavlek leg.; CBSS (Ar 3687) 3 우, 5 juvs; southern limit of Zavala village, Bjelušica ("Bjelušica

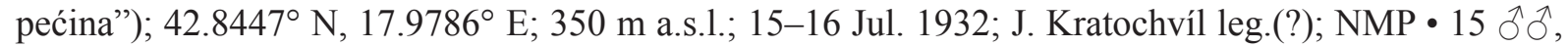
10 우; Zavala; $42.849^{\circ}$ N, $17.978^{\circ}$ E; 19 Sep. 1970; A. Senglet leg.; MHNG • 1 q; near monastery
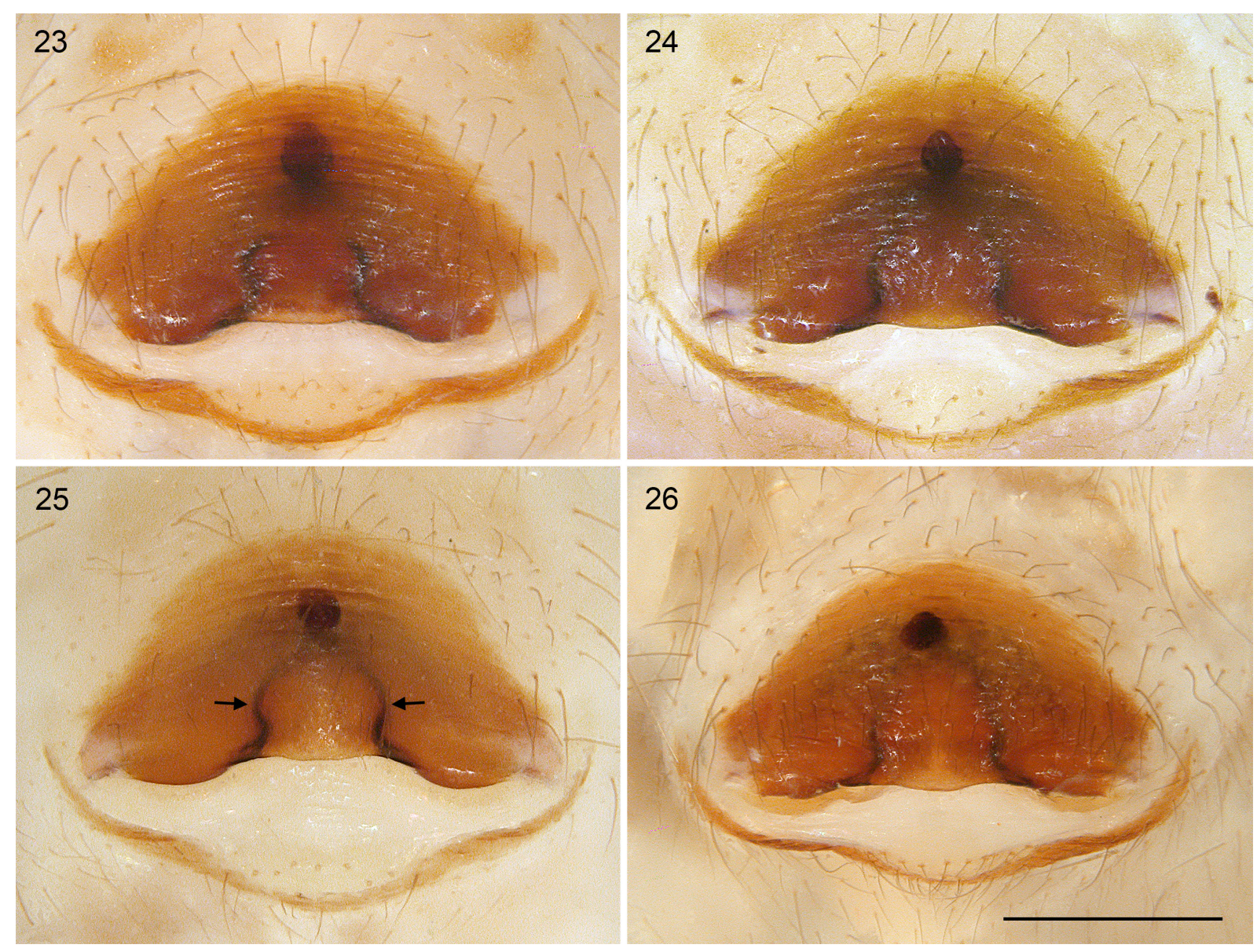

Figs 23-26. Stygopholcus absoloni (Kulczyński, 1914); epigyna, ventral views. 23. Balićeva špilja, Croatia (ZFMK (Ar 22195)). 24. Biokovo, Kukor, Croatia (CBSS (Ar 3367)). 25. Studenci (grotte), Bosnia and Herzegovina (MHNG); arrows point at distinctive internal sclerites. 26. Vjetrenica, Bosnia and Herzegovina (ZFMK (Ar 22192)). Scale bar: $0.5 \mathrm{~mm}$ (all at same scale). 

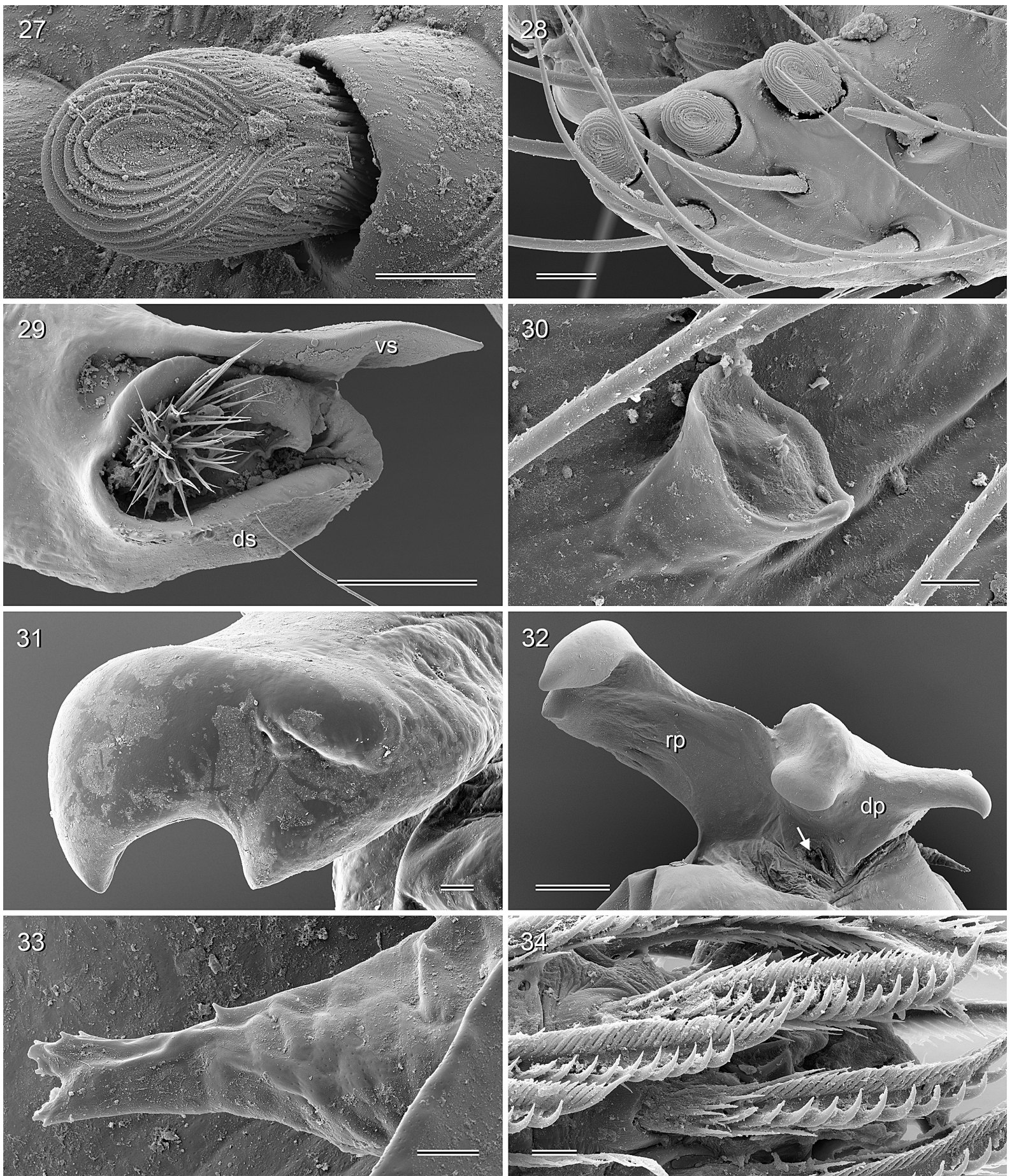

Figs 27-34. Stygopholcus absoloni (Kulczyński, 1914); male from Počitelj/Čapljina, Bosnia and Herzegovina (MHNG). 27. Modified hair frontally on male chelicerae. 28. Left male cheliceral apophysis with modified hairs. 29. Tip of left procursus, retrolateral view. 30. Male palpal tarsal organ. 31. Retrolateral bulbal process, distal view. 32. Bulbal processes; arrow points at opening of sperm duct. 33. Transparent bulbal process. 34. Comb hairs on left tarsus 4, prolateral view. Abbreviations: $\mathrm{dp}=$ dorsal process; $\mathrm{ds}=$ dorsal sclerite; $\mathrm{rp}=$ retrolateral process; $\mathrm{vs}=$ ventral sclerite. Scale bars: 27 , $30,33,34=10 \mu \mathrm{m} ; 28,31=20 \mu \mathrm{m} ; 29,32=100 \mu \mathrm{m}$. 
of Zavala, Pećina kod Monastira; 42.850 N, 17.979 E; 16 Jul. 1932; J. Kratochvíl leg.; NMP • 1 ○’; Zavala monastery, small cave behind monastery; $42.8499^{\circ} \mathrm{N}, 17.9795^{\circ} \mathrm{E} ; 300 \mathrm{~m}$ a.s.1.; 27 May 2014: B.A. Huber and M. Komnenov leg.; ZFMK (Ar 22193) • 1 \%, 1 o ; Zavala, Orlica pećina; $42.846^{\circ} \mathrm{N}$, $17.973^{\circ}$ E; 23 Oct. 1968; G. and A. Vigna leg.; MCVR • 1 §, 2 우; 2 km NW of Zavala, Grabova peć kod Grabova dola; $42.858^{\circ}$ N, 17.960 E; 20 Jul. 1932; J. Kratochvíl leg.; NMP • 1 đ, 1 ㅇ, with Kratochvíl's original label "Stygopholcus Absoloni (Kulcz.), + + et $\delta$ type! 19 b" [these are probably Kratochvíl's (1940) 'emended types' and the supposed syntypes in Růžička et al. (2005)]; presumably from same locality as preceding; NMP • 1 ก, 1 o; "Inv. No 409"; same collection data as as for preceding ("Grabowa-Höhle, leg. Kratochvíl 1932”); NHMW • 1 + , 1 juv.; Čvaljina, Zavala, Baba pećina; $42.8608^{\circ} \mathrm{N}, 17.9722^{\circ}$ E; $270 \mathrm{~m}$ a.s.l.; 27 Aug. 2017; M. Pavlek leg.; CBSS (Ar 5509) • 1 ○; same locality as for preceding; 2 Dec. 2014; M. Pavlek leg.; CBSS (Ar 3479) • 1 đ, 1 + , 5 juvs; between Grabova peć and Duga peć, Vukotina peć kod Čvaljinskih staja; $42.86^{\circ} \mathrm{N}, 17.96^{\circ} \mathrm{E}$; date unknown; J. Kratochvíl leg.(?); NMP • 1 đ, 1 क , 5 juvs; S of Zavala, near Belenići, Bezdan pod Licem; $42.813^{\circ} \mathrm{N}$, 18.000 E; 25 Jul. 1932; J. Kratochvíl leg.; NMP• 1 đ, 2 우, 1 juv.; Njavre, Donje Hrasno, Markova

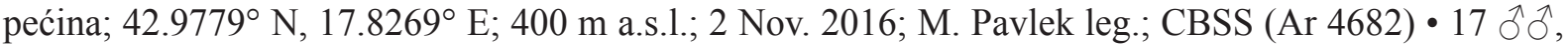

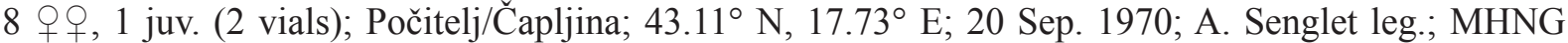

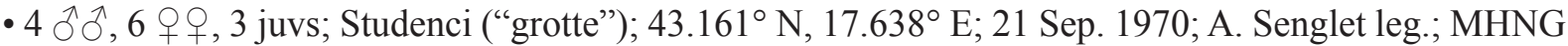

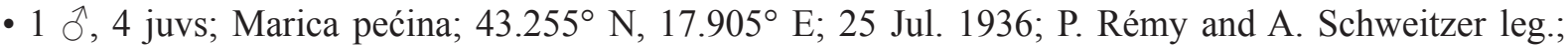

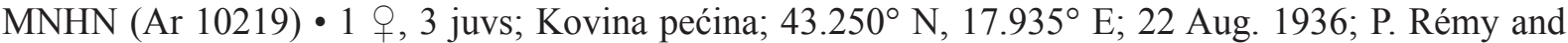

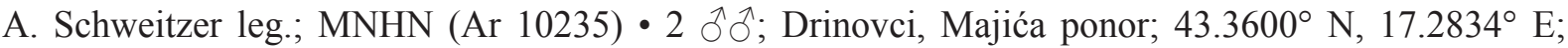
260 m a.s.l.; 5 Dec. 1999; M. Cukrov leg.; CBSS (Ar 3372) • 1 ô, 1 o; Grude, Peć Mlini, Ravlića pećina; $43.3390^{\circ} \mathrm{N}, 17.3233^{\circ}$ E; $250 \mathrm{~m}$ a.s.l.; 17 Jul. 1974; C. Deeleman leg.; NMNL (ARA 19022).

CROATIA - Split-Dalmatia • 1 đ̊; Lećevica, Kladnjice, Jama 7; $43.6918^{\circ}$ N, $16.2915^{\circ}$ E; $470 \mathrm{~m}$ a.s.1.; 28 Apr. 2020; T. Rožman leg.; CBSS (Ar 6962) • 1 ' ; same locality as for preceding; 17 Apr. 2020; N. Kuharić leg.; CBSS (Ar 6959) • 1 o ; Lećevica, Kladnjice, Trasirana jama; $43.6913^{\circ} \mathrm{N}, 16.2873^{\circ} \mathrm{E}$; 460 m a.s.l.; 17 Apr. 2020; T. Rožman leg.; CBSS (Ar 6980) • 1 đ’; Muć, Neorić, Velika špilja kod

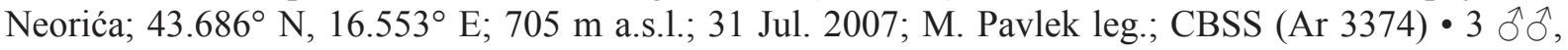
2 + ; ; Dugopolje, Balići, Balićeva špilja (= Kraljeva pećina); $43.5728^{\circ} \mathrm{N}, 16.5738^{\circ} \mathrm{E} ; 405 \mathrm{~m}$ a.s.1.; 31 Aug. 2000; F. Gasparo leg.; ZFMK (Ar 22195)・1 万; ; same locality as for preceding; 12 May 2012; M. Pavlek leg.; CBSS (Ar 3189)・ 1 क; same locality as for preceding; 5 Nov. 2016; M. Pavlek leg.; CBSS (Ar 4802) • 1 juv.; same locality as for preceding; date unknown; T. Delić et al. leg.; ZCSL (S19) • 1 ; ; Dugopolje, Balešina jama; 43.579 $\mathrm{N}, 16.581^{\circ}$ E; 310 m a.s.1.; 28 Apr. 2012; M. Pavlek leg.; CBSS (Ar 2759) 1 ○ं; Dugopolje, Kotlenice, Maklutača, in cave; $43.5693^{\circ} \mathrm{N}, 16.6205^{\circ} \mathrm{E} ; 400 \mathrm{~m}$ a.s.1.; 5 Nov. 2016; M. Pavlek leg.; CBSS (Ar 4978) • 1 ○ै, 2 우; Dugopolje, Kotlenice, Golubinka pećina; $43.56^{\circ}$ N, $16.65^{\circ}$ E; 2 May 1978; C. Deeleman leg.; AMNH • 1 क ; same collection data as for preceding; NMNL (ARA 19016) • 1 ð, 2 우, 3 juvs; Dugopolje, near Kotlenice, Vranjača špilja kod Dugopolja ("Vranjača pećina"); $43.5622^{\circ} \mathrm{N}, 16.6482^{\circ}$ E; 30 Jul. 1967; C. Deeleman leg.; NMNL (ARA 19020) • 1 juv., assigned tentatively; Dugopolje, Bradarići, Drinovčuša, in cave; $43.5485^{\circ} \mathrm{N}, 16.6386^{\circ} \mathrm{E} ; 795 \mathrm{~m}$ a.s.l.; 2 Nov. 2017; T. Delić leg.; CBSS (Ar 5698) • 2 우 (2 vials); Trilj, Ugljane, pećina Turkuša; $43.5789^{\circ} \mathrm{N}$, $16.7363^{\circ}$ E; 430 m a.s.1.; 16 Apr. 1974; C. Deeleman leg.; NMNL (ARA 19017-18) 1 ภ , 2 q ; Ugljane, Jama u Krčevini; $43.57^{\circ}$ N, $16.76^{\circ}$ E; 15 Apr. 1974; C. Deeleman leg.; NMNL (ARA 19019)

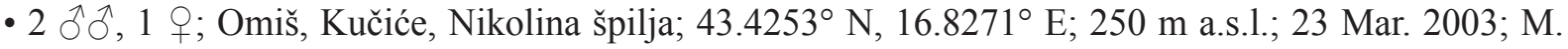
Cukrov leg.; CBSS (Ar 2003) • 2 ô, 1 क , 1 juv.; Omiš, Cetina (“Cajetina")-Canyon, small valley with stream, dense Fagus forest with Hedera sp., with big rocks, in crevices, under stones; 95 m a.s.l.; $43.433^{\circ}$ N, $16.762^{\circ}$ E; 13 May 2006; A. Schönhofer leg.; SMF • 2 đ̋ ${ }^{\lambda}, 2$ + + ; Šestanovac, Gornji Balići, Zlatna peć ("Balica zlatna pećina, Balići"); 43.490 N, 16.905 E; 17 Jun. 1974; C. Deeleman leg.;

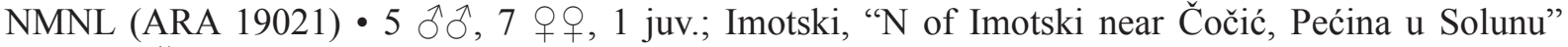
(possibly Špilja u Solunu; $43.4589^{\circ}$ N, $17.2217^{\circ}$ E); date unknown; J. Kratochvíl leg.(?); NMP • 1 ᄋ:

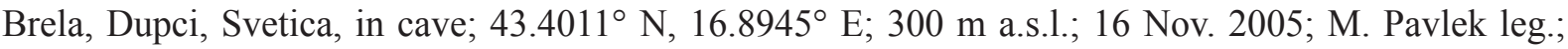



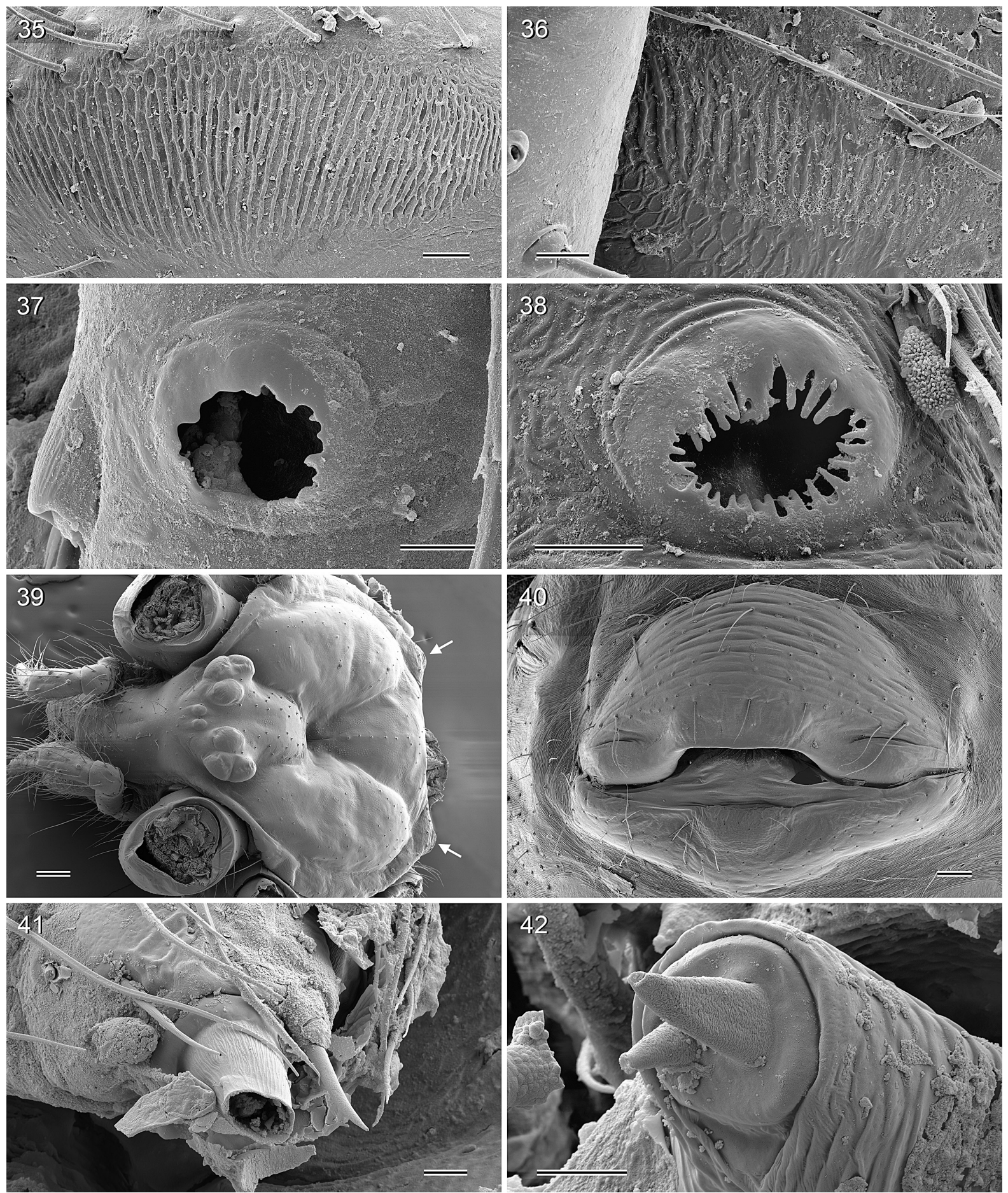

Figs 35-42. Stygopholcus absoloni (Kulczyński, 1914); male and female from Počitelj/Čapljina, Bosnia and Herzegovina (MHNG). 35-36. Stridulatory files on male and female chelicerae. 37. Female palpal tarsal organ. 38. Tarsal organ on female tarsus 2. 39. Female prosoma, oblique dorsal view; arrows point at posterior humps acting against modified areas on abdomen. 40. Epigynum, ventral view. 41. Female ALS. 42. Female PMS. Scale bars: $35=30 \mu \mathrm{m} ; 36=20 \mu \mathrm{m} ; 37-38=10 \mu \mathrm{m}, 39=200 \mu \mathrm{m}$; $40=100 \mu \mathrm{m} ; 41-42=10 \mu \mathrm{m}$. 
CBSS (Ar 2012) • 1 đ’; Brela, Nevistine stine, Jama iznad Prozorčića; $43.3838^{\circ} \mathrm{N}, 16.9320^{\circ} \mathrm{E}$; 615 m a.s.1.; 24 Mar. 2004; H. Bilandžija leg.; CBSS (Ar 1995) • 1 đ, 1 juv.; Šestanovac, Svaguše, Mužina špilja; $43.4170^{\circ} \mathrm{N}, 17.0076^{\circ}$ E; 505 m a.s.1.; 18 Oct. 2002; M. Lukić leg.; CBSS (Ar 1928)

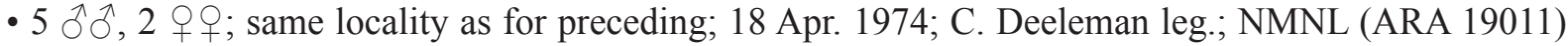

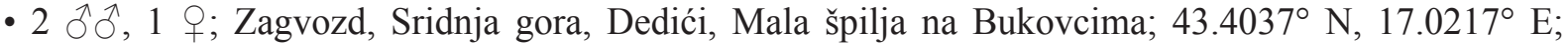
465 m a.s.l.; 21 Nov. 2005; M. Pavlek leg.; CBSS (Ar 2007) • 1 \%; Zagvozd, Rastovac, Dedići, Špilja u Radinovcima; 43,3873 N, 17.0103 E; 400 m a.s.1.; 25 Oct. 2006; M. Pavlek leg.; CBSS (Ar 2009)

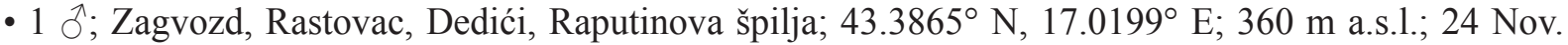
2005; M. Pavlek leg.; CBSS (Ar 2005) • 1 ○’; Zagvozd, Čikeši, Jama u Čikešima; $43.4161^{\circ} \mathrm{N}, 16.9670^{\circ} \mathrm{E}$; 200 m a.s.l.; 23 Nov. 2005; M. Pavlek leg.; CBSS (Ar 1996) • 1 q; Zagvozd, cave near Zagvozd; $43.40^{\circ} \mathrm{N}, 17.05^{\circ} \mathrm{E}$; 13 Oct. 2002; C. Deeleman leg.; CBSS (Ar 1930) • 1 Oे, 5 우; Zagvozd, Brnasi,

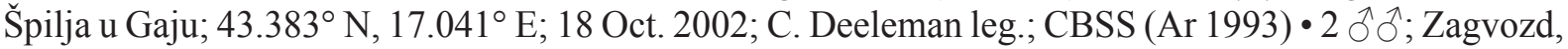
Prodani, Pružina špilja; $43.394^{\circ} \mathrm{N}, 17.059^{\circ} \mathrm{E} ; 520 \mathrm{~m}$ a.s.l.; 15 Oct. 2002; C. Deeleman leg.; CBSS (Ar 1926) • 1 q; Zagvozd, Spilja near Katušići; 43.384 N, 17.055 E; 18 Apr. 1974; C. Deeleman leg.; NMNL (ARA 19012) • 1 q, 1 juv.; Zagvozd, Župa, Brikinjava špilja; 43.3452 ${ }^{\circ} \mathrm{N}, 17.0969^{\circ} \mathrm{E}$; 740 m a.s.l.; 17 Nov. 2005; M. Pavlek leg.; CBSS (Ar 2000) • 1 đ̊; Zagvozd, Župa, Matijaševa peć; 43.3206 ${ }^{\circ} \mathrm{N}, 17.1223^{\circ}$ E; 585 m a.s.1.; 23 Jan. 2016; M. Lukić leg.; CBSS (Ar 3954)・ 1 q; same locality as for preceding; 23 Jan. 2016; M. Pavlek leg.; CBSS (Ar 3960) • 1 đ̂; Zagvozd, Župa, Gradska spila;

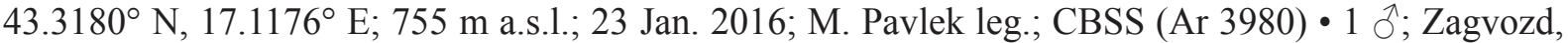
Župa, Samogorska špilja; 43.3182 ${ }^{\circ}$ N, $17.1211^{\circ}$ E; 715 m a.s.1.; 23 Jan. 2016; M. Pavlek leg.; CBSS (Ar 3935) • 1 đ̄; same locality as for preceding; 23 Jan. 2016; M. Lukić leg.; CBSS (Ar 3962)・ 1 juv.; same locality as for preceding; 23 Jun. 2017; R. Ozimec leg.; CBSS (5477) • 1 Ô, 1 q; Zagvozd, Župa, Stonjska peć donja; $43.3152^{\circ} \mathrm{N}, 17.1307^{\circ} \mathrm{E} ; 610 \mathrm{~m}$ a.s.1.; 22 Jan. 2016; M. Pavlek leg.; CBSS (Ar 3975) - 1 ô, 1 ㅇ, 1 juv.; Baška Voda, Bast, Kukor, in cave; 43.3556 ${ }^{\circ}$ N, $16.9917^{\circ}$ E; 425 m a.s.1.; 2 Jan. 1999;

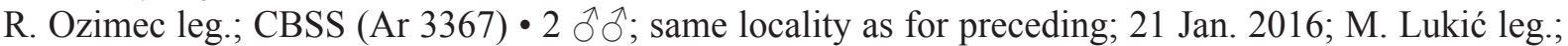
CBSS (Ar 3952) • 1 ' ; same locality as for preceding; 21 Jan. 2016; M. Pavlek leg.; CBSS (Ar 3968) - 3 우, 1 juv.; same locality as for preceding; 21 Jan. 2016; T. Rožman leg.; CBSS (Ar 3971) • 4 juvs, assigned tentatively; Makarska, Stipkovac, Jama pod Stipkovcem; $43.3090^{\circ} \mathrm{N}, 17.0651^{\circ} \mathrm{E} ; 1360 \mathrm{~m}$ a.s.1.; 21 Jun. 2017; A. Kirin leg.; CBSS (Ar 5467) • 1 9, 1 juv.; Tučepi, Ravna Vlaška, Tučepska Vilenjača ("Pećina Vilenjača"); $43.2854^{\circ} \mathrm{N}, 17.0842^{\circ}$ E; 1180 m a.s.l.; 28 Aug. 2000; F. Gasparo leg.; ZFMK (Ar 22194) • 1 q; same locality as for preceding; 30 Apr. 2012; A. Ćukušić leg.; CBSS (Ar 3370) • 1 q; same locality as for preceding; 24 Jun. 2017; M. Pavlek leg.; CBSS (5035) • 1 Õ, 2 오, 1 juv.; Tučepi, Ravna Vlaška, Jama kod Tučepske Vilenjače; $43.2847^{\circ} \mathrm{N}, 17.0850^{\circ} \mathrm{E} ; 1195 \mathrm{~m}$ a.s.1.; 17 Jun. 2002; R. Ozimec leg.; CBSS (Ar 2001) • 1 ô, 1 juv.; Vrgorac, Kozica, Jujnovići, Jujnovića šplija; $43.2666^{\circ}$ N, 17.1980 E; 405 m a.s.1.; 27 Apr. 2002; R. Ozimec leg.; CBSS (Ar 1999) • 3 q $q$; same locality as for preceding; 14 Oct. 2002; C. Deeleman leg.; CBSS (Ar 1927) • 1 o ; same locality as for preceding; 14 Oct. 2002; R. Ozimec leg.; CBSS (Ar 2006) • 1 क; same locality as for preceding; 1 May 2012; M. Pavlek leg.; CBSS (Ar 2773) • 1 q; Vrgorac, Saranač, Šošići, Jama iznad Saranača; $43.2490^{\circ}$ N, 17.1747 E; 770 m a.s.1.; 25 Mar. 2003; R. Ozimec leg.; CBSS (Ar 1998) • 1 đ, 1 \&, 7 juvs; probably same locality as for preceding; ["grotje bij Saranač op berg Skok", Gornje Igrane]; 7 Jul. 1974; C. Deeleman leg.; NMNL (ARA 19010) • 1 §̂, 1 o; Vrgorac, Zavojane, Signalka, in cave; $43.2691^{\circ}$ N, 17.2380 E; 1030 m a.s.1.; 29 Aug. 2018; T. Rožman leg.; CBSS (Ar 6573) • 1 đ , 3 juvs; Vrgorac, Zavojane, Rođendanska jama; $43.2686^{\circ} \mathrm{N}, 17.2389^{\circ} \mathrm{E} ; 1045 \mathrm{~m}$ a.s.1.; 29 Apr. 2018; F. Belak leg.; CBSS (Ar 6420) • 1 §, 2 juvs; Vrgorac, Zavojane, Škrapica, in cave; $43.2558^{\circ} \mathrm{N}, 17.2679^{\circ} \mathrm{E} ; 720 \mathrm{~m}$ a.s.1.; 30 Apr. 2018; F. Belak leg.; CBSS (Ar 6578) • 2 + ; ; Vrgorac, Baletići, Ančića velika Golubinka, in cave; 43.2584 N, $17.2842^{\circ}$ E; 875 m a.s.1.; 8 Oct. 2018; N. Kuharić leg.; CBSS (Ar 6553) • 1 ○, 1 q; Vrgorac, Zavojane, Drž gače 2, in cave; $43.2590^{\circ} \mathrm{N}, 17.2828^{\circ} \mathrm{E} ; 870 \mathrm{~m}$ a.s.1.; 8 Oct. 2018; N. Sudar and N.

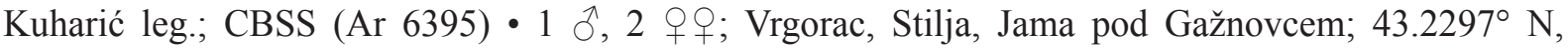
$17.3142^{\circ}$ E; $510 \mathrm{~m}$ a.s.1.; 4 Nov. 2016; M. Pavlek leg.; CBSS (Ar 4283) • 3 + $\odot, 1$ juv.; same locality as for preceding; 31 Mar. 2018; F. Belak leg.; CBSS (Ar 6587) • 1 ô, 1 q, 1 juv. (2 vials); Vrgorac, Orah 
[hill], Ružovača pećina; 43.229 N, 17.417 E; 390 m a.s.1; 23 Oct. 1980 and 26 Apr. 1981; C. Deeleman leg.; NMNL (ARA 19008-9) • 1 क , 1 juv.; Hvar Island, Jelsa, Viskoka, Franetova jama; 43.14 N, $16.93^{\circ}$ E; 28 Feb. 1999; R. Ozimec leg.; CBSS (Ar 3363) • 1 o; Hvar Island, Jelsa, Zastražišče, Vela Stiniva cove, Špilja u Grliću; 43.1580 N, $16.8136^{\circ}$ E; 3 m a.s.1; 3 May 2011; D. Hmura leg.; CBSS (Ar 2513) • 2 ○ึ; same locality as for preceding; 19 Aug. 2016; M. Pavlek leg.; CBSS (Ar 3996) • 1 ㅇ; Hvar Island, Jelsa, Humac, Grapčeva špilja; 43.1341 ${ }^{\circ}$ N, 16.7537 E; 200 m a.s.1.; 3 May 2011; A. Ćukušić leg.; CBSS (Ar 2512) •1 §̂, 1 ㅇ (epigynum missing); same locality as for preceding, "Grabćina

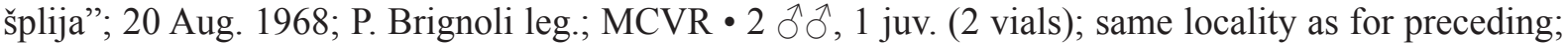
26 Oct. 1980 (one vial without date); C. Deeleman leg.; NMNL (ARA 19013-14). - DubrovnikNeretva 1 q, 1 juv.; Ploče, Gradac, Ričevica, in cave; $43.0999^{\circ}$ N, $17.3909^{\circ}$ E; 525 m a.s.1.; 5 Nov. 2019; T. Rožman leg.; CBSS (Ar 6430) • 1 o, 1 juv.; Kula Norinska, Krvavac, Kokanova jama;

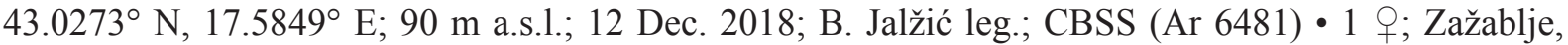
Goračić, Vidonje, Šolkina jama; 42.9665 N, 17.6625 E; 230 m a.s.l.; 27 Feb. 2016; B. Jalžić leg.; CBSS (Ar 3989) • 2 우; Zažablje, Dobranje, Kornjatuša, in cave; $42.9790^{\circ}$ N; $17.6740^{\circ}$ E; 610 m a.s.l.; 2 Nov. 2013; T. Čuković leg.; CBSS (Ar 3384) • 1 +; Dubrovačko Primorje, Slano, Točionik, Vranja peć; $42.8683^{\circ} \mathrm{N}, 17.8124^{\circ}$ E; 230 m a.s.l.; 2 Nov. 2016; T. Rožman leg.; CBSS (Ar 5148) • 1 q; same locality as for preceding; 2 Nov. 2006; M. Pavlek leg.; CBSS (Ar 4877) • 1 §, 1 ○; Pelješac, Ston, Ponikve, Špilja Crno jezero; $42.8473^{\circ}$ N, $17.6365^{\circ}$ E; 415 m a.s.1.; 15 Nov. 2018; N. Kuharić leg.; CBSS (Ar 6276) • 1 '; same collection data as for preceding; CBSS (Ar 6284) 1 q ; Pelješac, Ston, Putniković, Kameni kolačić, in cave; $42.8731^{\circ}$ N, $17.4664^{\circ}$ E; 420 m a.s.1.; 28 Mar. 2019; N. Kuharić leg.; CBSS (Ar 6161) • 1 क; Pelješac, Janjina, Vardišta, Špilja u Žarmi; $42.9354^{\circ} \mathrm{N}, 17.4382^{\circ}$ E; 35 m a.s.1.; 28 Apr. 2019; T. Rožman leg.; CBSS (Ar 6258) • 1 đ̂, 1 juv.; same collection data as for preceding; M. Pavlek leg.; CBSS (Ar 6261) • 1 đं; Pelješac, Orebić, Oskorušno, Špilja kod Zagruda u vinogradu; $42.9666^{\circ} \mathrm{N}$, 17.3227 E; 295 m a.s.1.; 13 Dec. 2019; M. Pavlek leg.; CBSS (Ar 6464) • 1 P; Pelješac, Orebić, below monastery, Čagjina jama; $42.9771^{\circ}$ N, $17.1552^{\circ}$ E; 115 m a.s.1.; 14 Nov. 2018; F. Šarc leg.; CBSS (Ar 6247) • 1 क; Pelješac, Orebić, Kućište, Šimunkovića špilja; $42.9786^{\circ} \mathrm{N}, 17.1291^{\circ}$ E; 50 m a.s.1.; 3 Nov. 2016; M. Pavlek leg.; CBSS (Ar 4692) • 1 क , 2 juvs; same locality as for preceding; 26 Apr. 2019; T. Rožman leg.; CBSS (Ar 6781) • 1 ơ; Pelješac, Trpanj, Duba Pelješka, Spilja Rupine; $43.0053^{\circ}$ N, $17.1580^{\circ}$ E; 460 m a.s.1.; 6 Apr. 2019; T. Dražina leg.; CBSS (Ar 5980) • 1 q; same locality as for preceding; 24 Apr. 2019; M. Pavlek leg.; CBSS (Ar 5986) • 1 đ; same locality as for preceding; 24 Apr. 2019; T. Rožman leg.; CBSS (Ar 6197) • 1 đో; Pelješac, Trpanj, Donja Vrućica, Špilja u Privoru; 43.0092 ${ }^{\circ}$ N, $17.2133^{\circ}$ E; 180 m a.s.1.; 26 Mar. 2019; N. Kuharić leg.; CBSS (Ar 6038) • 1 q, 1 juv.;

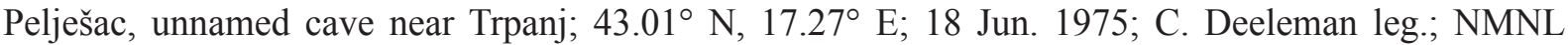
(ARA 19015) • 1 đ ; Korčula, Žrnovo, Deukalion špilja; $42.9557^{\circ} \mathrm{N}, 17.0976^{\circ} \mathrm{E}$; $110 \mathrm{~m}$ a.s.1.; 21 Mar. 2010; R. Ozimec leg.; ROC (R97).

Assigned tentatively (see Variation)

CROATIA - Split-Dalmatia - 1 đ̄; Baška Voda, Bast, Topići, Jama na Brezdanu; $43.3670^{\circ} \mathrm{N}$, 16.9732 ${ }^{\circ}$ E; 915 m a.s.1.; 20 Mar. 2004; collector unknown; CBSS (Ar 1994).

Credible published records (not examined or juveniles only) BOSNIAAND HERZEGOVINA • 3 juvs; near Čvaljina, Duboka peć; $42.87^{\circ} \mathrm{N}, 17.97^{\circ} \mathrm{E}$; date unknown;

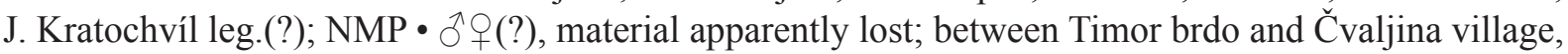
Duga peć; $42.87^{\circ} \mathrm{N}, 17.95^{\circ} \mathrm{E}$; date unknown; J. Kratochvíl leg.(?) [Kratochvíl 1940] • đ̊ $q(?)$, material apparently lost; Baba pećina; $42.8608^{\circ} \mathrm{N}, 17.9722^{\circ} \mathrm{E}$; $1932(?) ; \mathrm{J}$. Kratochvíl leg.(?) [Kratochvíl 1940] - đo (?), material apparently lost; $500 \mathrm{~m} \mathrm{~W}$ of "Bjelušica pećina", env. Zavala, Orlica pećina; $42.846^{\circ} \mathrm{N}$, $17.973^{\circ}$ E; 1932(?); J. Kratochvíl leg.(?) [Kratochvíl 1940] • 1 juv.; S of Zavala, near Belenići, Jama za Jamskim vrhom; $42.81^{\circ} \mathrm{N}, 18.00^{\circ}$ E; 28 Jul. 1932; J. Kratochvíl leg.(?); NMP. 
CROATIA - Split-Dalmatia - đq(?), material apparently lost; Muć, near Sinj, Pećina kod Sinja; $43.70^{\circ} \mathrm{N}, 16.64^{\circ} \mathrm{E} ; 1932(?) ; \mathrm{J}$ Kratochvíl leg.(?) [Kratochvíl 1940] • đo (?), material apparently lost; Dugopolje, near Kotlenice, Vranjača špilja kod Dugopolja ("Vranjača pećina"); $43.5622^{\circ} \mathrm{N}, 16.6482^{\circ} \mathrm{E}$; 445 m a.s.l.; 1932(?); J. Kratochvíl leg.(?) [Kratochvíl 1940] • ^̊ $O$ (?), material apparently lost; Dugopolje, near Dugopolje, Kraljeva pećina [Kratochvíl 1940 used this present-day synonym of Balićeva špilja for a cave "near" Balićeva špilja]; 43.573 N, 16.570 E, 1932(?); J. Kratochvíl leg.(?) [Kratochvíl 1940] - đof(?), material apparently lost; Dugopolje, near Dugopolje, Balićeva špilja; $43.573^{\circ} \mathrm{N}, 16.570^{\circ} \mathrm{E}$; 405 m a.s.l.; 1932(?); J. Kratochvíl leg.(?) [Kratochvíl 1940] • đ̊ $+(?)$, material apparently lost; Hvar Island, Jelsa, Grapčeva špilja (“Grabćina šplija”); 43.1341 N, $16.7537^{\circ}$ E; 1932(?); J. Kratochvíl leg. (?) [Kratochvíl 1940].

\section{Redescription}

Male (Zavala, MHNG)

MEASUREMENTS. Total body length 5.8, carapace width 2.2. Distance PME-PME $150 \mu \mathrm{m}$; diameter PME $140 \times 180 \mu \mathrm{m}$; distance PME-ALE $30 \mu \mathrm{m}$; diameter AME $65 \mu \mathrm{m}$; distance AME-AME $25 \mu \mathrm{m}$. Leg 1: $55.2(14.8+1.1+14.0+22.1+3.2)$, tibia $2: 10.8$, tibia 3: 8.7, tibia 4: 9.5 ; tibia $1 \mathrm{~L} / \mathrm{d}: 62$.

COLOR (in ethanol). Carapace pale whitish to ochre-yellow, ocular area and clypeus slightly darker (light brown); sternum light brown, with darker brown radial marks; legs ochre yellow, without dark rings, without dark lines; abdomen monochromous pale gray, only above spinnerets with darker mark.

Body. Habitus as in Fig. 1. Ocular area slightly raised. Carapace with deep median pit and pair of very shallow indistinct furrows diverging from pit to posterior margin of carapace. Clypeus unmodified. Sternum wider than long (1.50/1.00), unmodified. Abdomen oval, pointed at spinnerets.

Chelicerae. As in Figs 19-20, with pair of large frontal apophyses set with 2 and 3 modified hairs, respectively, with 17 and 18 modified hairs each on frontal face; with distinct stridulatory ridges (Fig. 35).

PALPS. In general as in S. skotophilus (cf. Figs 6-8); coxa with retrolateral hump; trochanter barely modified; femur with distinct retrolateral process proximally, with stridulatory pick (modified hair) proximally on prolateral side; femur-patella joints shifted towards prolateral; tibia very large, with two trichobothria; tibia-tarsus joints shifted towards retrolateral; procursus (Figs 12-13) slightly curved towards ventral, dorsally with curved hairs (distal third curved 'upwards'), with strong hump on prolateral side (asterisk in Fig. 12). Procursus tip with evenly curved dorsal sclerite and strong hinged ventral sclerite (ds and vs in Figs 13, 29); with strong conical process on prolateral side bordering hinged sclerite (arrow in Fig. 14); membranous central part on retrolateral side with brush of hair-like processes (Fig. 29). Genital bulb (Figs 15-18) with basal sclerite connecting to tarsus (bs in Fig. 15), small transparent dorsal process (arrow in Fig. 17; Fig. 33), and main sclerite consisting of long retrolateral process and short dorsal process ( $\mathrm{rp}$ and dp in Figs 15-18, 32); retrolateral process with bifid tip, distal part on ventral side thickened and heavily sclerotized; dorsal process with finger-shaped ventral tip slightly hooked, retrolateral tip wider, with additional strong protrusion on ventral side.

LEGS. With short spines ventrally on femur 1 in two rows, prolateral row $\sim 19$ spines, retrolateral row $\sim 24$ spines; without curved hairs; few vertical hairs; retrolateral trichobothrium of tibia 1 at 3\%; prolateral trichobothrium absent on tibia 1, present on other tibiae; tarsus 1 with irregular platelets rather than with distinct pseudosegments.

Male (variation)

Most males with monochromous abdomen with one or few dark marks above spinnerets; few males with additional dark marks laterally in posterior third. Tibia $1(\mathrm{~N}=60)$ : 10.4-15.1 (mean 12.8); tibia 2 
$(\mathrm{N}=64)$ : 7.6-11.5 (mean 9.6); spines on femur $1(\mathrm{~N}=27)$ : 0-48 (mean 38); modified hairs on cheliceral basis $(\mathrm{N}=122)$ : 11-23 (mean 17.4); modified hairs on cheliceral apophysis $(\mathrm{N}=122)$ : 1-4 (mean 2.6); chelicerae maximum width $(\mathrm{N}=61)$ : $0.66-0.85$ (mean 0.78$)$; procursus length $(\mathrm{N}=58)$ : $1.10-1.28$ (mean 1.21); bulbal measures A-E: A $(\mathrm{N}=28)$ : 0.31-0.37 (mean 0.35); $\mathrm{B}(\mathrm{N}=28)$ : 0.06-0.08 (mean $0.07) ; \mathrm{C}(\mathrm{N}=20): 0.22-0.28$ (mean 0.26); $\mathrm{D}(\mathrm{N}=20)$ : 0.18-0.23 (mean 0.21); $\mathrm{E}(\mathrm{N}=22): 0.68-0.77$ (mean 0.73); epiandrous spigots $(\mathrm{N}=20)$ : 5-7 (mean 6.1).

In the single male from Jama na Brezdanu (Croatia), the bulb differs in several respects from all other material examined, but it is unclear if this is teratological or not (even though it is symmetric). Most conspicuously, the heavy sclerite connecting the basal and distal bulbal sclerites (asterisk in Fig. 18) is much smaller and it is not connected to the basal bulbal sclerite; bulb measure $\mathrm{E}$ also deviates strongly (asterisk in Fig. 160).

\section{Female}

In general similar to male, but femur 1 without spines, with pair of indistinct processes posteriorly on carapace (arrows in Fig. 39) and pair of opposing poorly visible plates on abdomen; distance between processes/plates: 1.1. Tibia $1(\mathrm{~N}=55)$ : 8.2-15.1 (mean 12.0); tibia $2(\mathrm{~N}=59)$ : 4.4-11.1 (mean 8.6); epigynum width $(\mathrm{N}=57)$ : $0.72-1.22$ (mean 1.05); epigynum length $(\mathrm{N}=57)$ : $0.42-0.68$ (mean 0.54 ); epigynal measure $\mathrm{F}(\mathrm{N}=57)$ : $0.21-0.37$ (mean 0.30). Epigynum (Figs 23-26) anterior plate semicircular, weakly protruding, with weak transversal furrows, with internal structures visible in uncleared specimens: round anterior structure and parallel sclerites posteriorly; posterior plate short but wide. Internal genitalia (Figs 21-22, 145-146) with distinctive ventral arc (va in Fig. 153; relatively shorter and wider than in other species), with large pore plates (Fig. 22).

\section{Distribution}

Known from southern Croatia and southern Bosnia and Herzegovina (Fig. 168).

Stygopholcus skotophilus Kratochvíl, 1940

Figs 2, 6-8, 43-76, 147-148, 154

Stygopholcus skotophilus Kratochvíl, 1940: 16, figs 1a, c, 2, 4c-d, 5c-d, 6, 7 (right).

Holocnemus (Hoplopholcus) Absolonii Kulczyński, 1914: 355 (part), pl. 16, fig. 25 only.

Holocnemus (Stygopholcus) absoloni (misidentification) - Kratochvíl 1935: 11.

Stygopholcus skotophilus - Senglet 1971: figs 40-41; 2001: 65, fig. 22.

Hoplopholcus scotophilus - Brignoli 1971: 261, fig. 19. - Tomić et al. 2000: 35P (specimen from Vidrovan cave).

Stygopholcus skotophilus montenegrinus (misidentification) - Naumova et al. 2016: 434 (all specimens except those from Cetinje, Golubova pećina, and Babatuša pećina).

"S112 Stygopholcus absoloni? Ba115" \& "S108 Stygopholcus absoloni? Bal8" - Eberle et al. 2018. Huber et al. 2018.

\section{Misidentification}

Hoplopholcus scotophilus - Tomić et al. 2000: 35P (specimens from near Velja cave; see S. montenegrinus).

\section{Diagnosis}

Distinguished from other species in the 'northern clade' (S. absoloni; S. montenegrinus) by bulb measures A and C (Fig. 158; smaller than other species, no overlap), by bulb measure D (Fig. 159; larger 

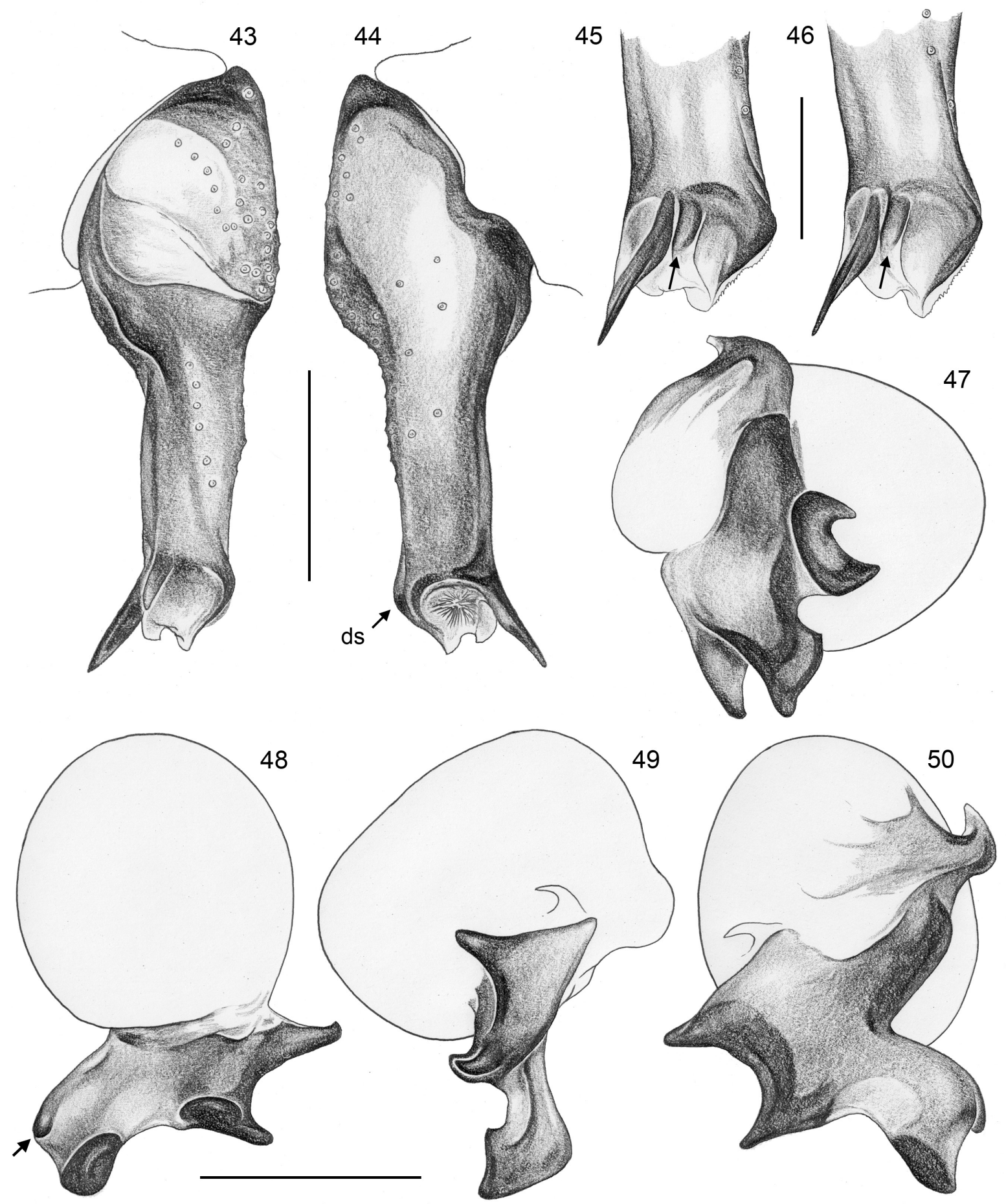

Figs 43-50. Stygopholcus skotophilus Kratochvíl, 1940. 43-44. Male from Ilijina pećina, Bosnia and Herzegovina (ZFMK (Ar 22198)), left tarsus and procursus, prolateral and retrolateral views. 4546. Males from Pećina u Ivici, Montenegro (ZFMK (Ar 22196)) and from Grabova pećina, Sedlari, Bosnia and Herzegovina (MHNG), tip of left procursus, prolateral (slightly ventral) views; arrows point at distinctive prolateral sclerites. 47-50. Male from Ilijina pećina (ZFMK (Ar 22198)), left genital bulb, retrolateral, ventral, prolateral-distal, and dorso-distal views; arrow points at wart that is more strongly developed in specimens from near Sedlari. ds $=$ dorsal sclerite. Scale bars: $43-44,47-50=0.5 \mathrm{~mm}$; $45-46=0.25 \mathrm{~mm}$. 
than other species, with little overlap), and by epigynal measure F (Fig. 164; larger than other species, with little overlap); from $S$. absoloni also by fewer hairs on male cheliceral base (Figs 51, 162; with little overlap), by smaller, weakly sclerotized prolateral sclerite on procursus (arrows in Figs 45-46), by straight procursus (rather than curved toward ventral; Fig. 44), by longer procursus (Fig. 161; with little overlap), by shape of retrolateral bulbal process in retrolateral view (Fig. 47; large notch on ventral side), and by bulb measures B and E (Figs 157, 160; larger than in S. absoloni, with little overlap); from S. montenegrinus also by wider prolateral sclerite on procursus (arrows in Figs 45-46);

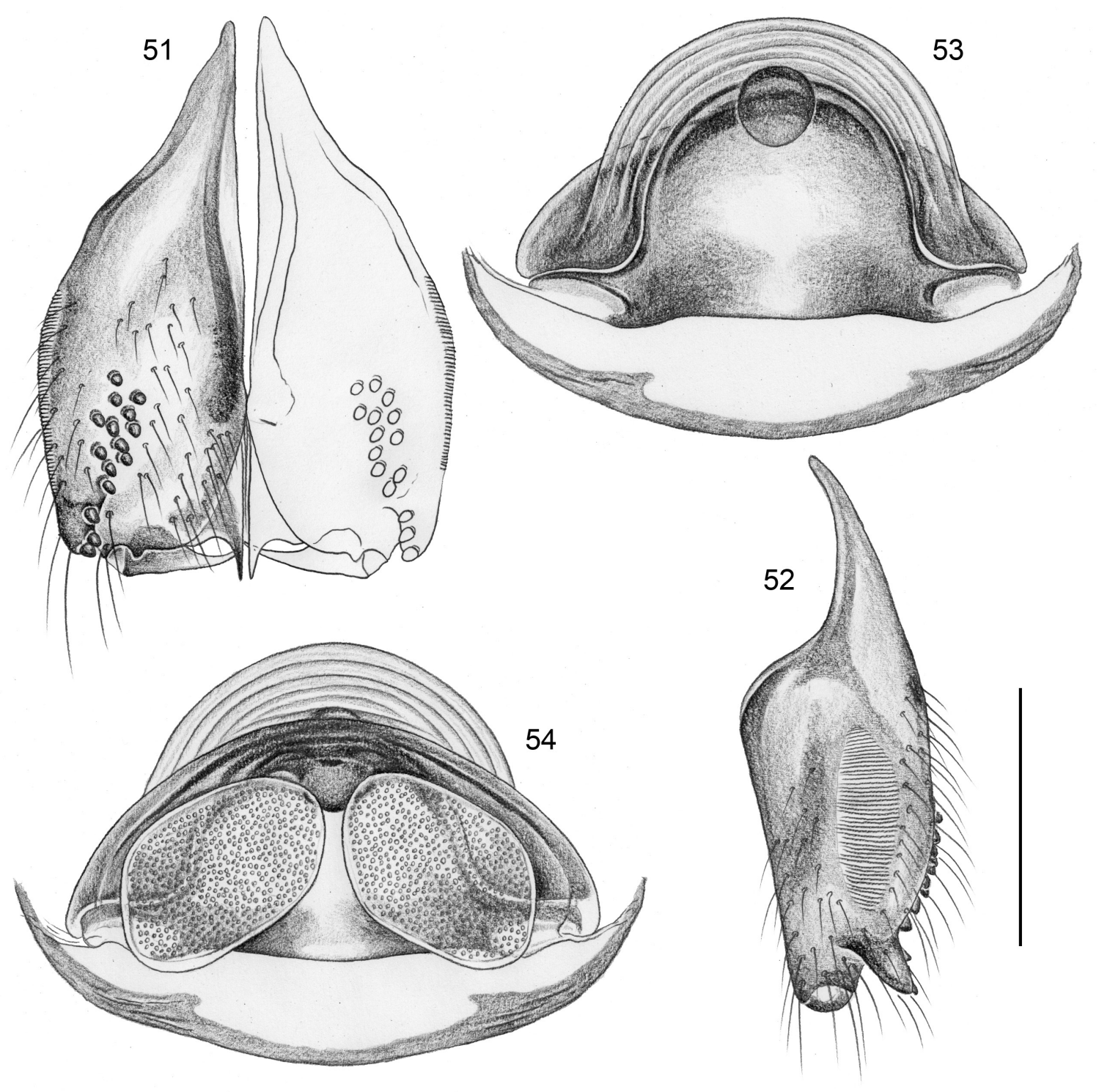

Figs 51-54. Stygopholcus skotophilus Kratochvíl, 1940; male from Ilijina pećina, Bosnia and Herzegovina (ZFMK (Ar 22198)), female from Pećina u Ivici, Montenegro (ZFMK (Ar 22196)). 5152. Male chelicerae, frontal and lateral views. 53-54. Cleared female genitalia, ventral and dorsal views. Scale bars: $0.5 \mathrm{~mm}$. 


\section{Type material}

MONTENEGRO - Kotor $\bullet 1$ ð , 1 ( without epigynum), 5 juvs, 1 prosoma (juv.?), 1 damaged abdomen (juvenile?), one complete juvenile abdomen, putative syntypes (see Remark below), examined; Pećina u Ivici; $42.5384^{\circ} \mathrm{N}, 18.6207^{\circ} \mathrm{E}$ [Naumova et al. 2019: $42.569^{\circ} \mathrm{N}, 18.662^{\circ} \mathrm{E}$ ]; date and collector unknown; NMP.

\section{Remark}

According to Rủžička et al. (2005), the type vial originally contained two labels saying "Stygopholcus skotophilus sp. n." and "Pećina u Ivici, č. III". This agrees with other labels in Kratochvíl's handwriting in the NMP Stygopholcus material. Both labels are lost. Rủžička et al. (2005) further state that the vial contained " $1 \delta 3 q$ (one of them without epigyne) 4 juv.", suggesting that there were two epigyna. However, the vial does not contain any epigynum.

\section{Other material examined}

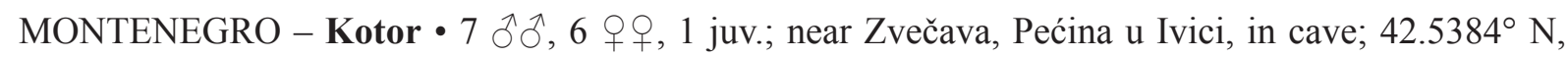
$18.6207^{\circ}$ E; 960 m a.s.1.; 28 May 2014; B.A. Huber and M. Komnenov leg.; ZFMK (Ar 22196) • 1 ô, 1 +, 3 juvs, in pure ethanol; same collection data as for preceding; ZFMK (Bal15) 9 $\widehat{\partial}, 7$ q $q$; near Zvečava, near Pećina u Ivici, under stones in forest; $42.5349^{\circ} \mathrm{N}, 18.6237^{\circ} \mathrm{E} ; 1000 \mathrm{~m}$ a.s.1.; 28 May 2014; B.A. Huber and M. Komnenov leg.; ZFMK (Ar 22197) • 2 9o, 1 juv., in pure ethanol; same collection data as for preceding; ZFMK (Bal16) 1 o ; Crkvice, Vilina pećina; $42.5546^{\circ} \mathrm{N}, 18.6624^{\circ} \mathrm{E}$ [Naumova et al. 2019: $42.560^{\circ} \mathrm{N}, 18.629^{\circ}$ E]; 855 m a.s.1.; 30 Oct. 2015; M. Pavlek leg.; CBSS (Ar 3674) - 1 juv.; same locality as for preceding; 17 Jul. 1973; C. Deeleman leg.; NMNL (ARA 19032) • 1 万̃, 1 ; Crna Gora, Dragalj, Pećina kod Dvoriškog ždrijela; $42.5881^{\circ} \mathrm{N}, 18.6737^{\circ} \mathrm{E} ; 715 \mathrm{~m}$ a.s.1.; 3 Dec. 2014; M. Pavlek leg.; CBSS (Ar 3482) • 1 ô, 3 q $ᄋ$; Crni Nugli, unnamed cave; $42.586^{\circ} \mathrm{N}, 18.697^{\circ} \mathrm{E}$;

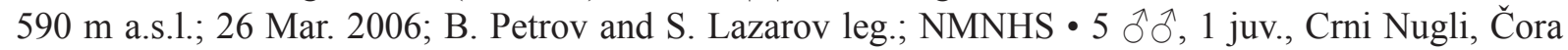
pećina; $42.594^{\circ} \mathrm{N}, 18.712^{\circ}$ E; $750 \mathrm{~m}$ a.s.1.; 26 Mar. 2006; B. Petrov and S. Lazarov leg.; NMNHS • 1 ठૈ, 1 juv.; Crni Nugli, Dragalj, Pećina s mostom; $42.5929^{\circ}$ N, $18.7100^{\circ}$ E; 725 m a.s.1.; 25 Aug. 2017; M. Pavlek leg.; CBSS (Ar 5387) • 1 क; same locality as for preceding; 25 Aug. 2017; A. Kirin leg.; CBSS (Ar 5391) • 1 क; Crni Nugli, Dragalj, Trokutasta pećina; $42.5931^{\circ} \mathrm{N}, 18.7107^{\circ} \mathrm{E} ; 710 \mathrm{~m}$ a.s.1.; 25 Aug. 2017; A. Kirin leg.; CBSS (Ar 5395) • 1 đ; 4 km SW Crni Nugli, Miljukovica pećina [Kratochvíl 1935: "Mijukovica"]; $42.57^{\circ}$ N, $18.67^{\circ}$ E; 1932(?); J. Kratochvíl leg.(?); NMP • 1 + ; Risan, Teveričke Polje, Teverička pećina; $780 \mathrm{~m}$ a.s.l.; $42.570^{\circ} \mathrm{N}, 18.676^{\circ} \mathrm{E}$; 28 Jul. 2010; M. Komnenov leg.; CMK • 1 \%; Risan, Kameno More, Knezlaz, Bukovica pećina; $42.545^{\circ}$ N, $18.683^{\circ}$ E; 1 Aug. 2010; M. Komnenov leg.; CMK • 2 o $\odot$; Risan, Kameno More, Gornja Pokljuka pećina; $42.542^{\circ} \mathrm{N}, 18.693^{\circ} \mathrm{E} ; 580 \mathrm{~m}$ a.s.1.; 31 Jul. 2010; M. Komnenov leg.; CMK・ 7 $\partial^{\lambda}, 5$ 우, 2 juvs; same locality as for preceding; 27 Mar.

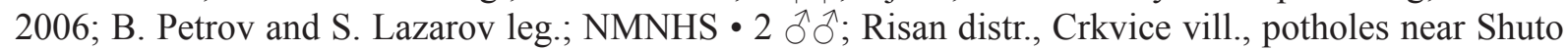
Blagojević monastery; $42.568^{\circ} \mathrm{N}, 18.623^{\circ} \mathrm{E} ; 1045 \mathrm{~m}$ a.s.1.; 17 Aug. 2006; B. Petrov and S. Lazarov leg.; NMNHS • $1{ }^{\top}$; Blagojevići, Ovnova rupa, in cave; $42.5681^{\circ} \mathrm{N}, 18.6298^{\circ} \mathrm{E} ; 1040 \mathrm{~m}$ a.s.1.; 25 Aug. 2017; M. Pavlek leg.; CBSS (Ar 5384) • 2 సึ ${ }^{\lambda}, 3$ juvs; Strmena pećina, selo Han, 2 km E of Bor pećina, near Veli Vrh; $42.564^{\circ} \mathrm{N}, 18.633^{\circ}$ E; 28 Jul. 1969; J. Kratochvíl leg.; NMNL (ARA 19030) • 1 q; Risan, Sopot pećina; $42.5137^{\circ} \mathrm{N}, 18.6816^{\circ}$ E; 13 Jul. 1971; C. Deeleman leg; NMNL (ARA 19031). - Nikšíć - 1 ○ं; Grahovo, Pećina Vojvode Dakovića; $42.6558^{\circ}$ N, $18.6758^{\circ}$ E; 680 m a.s.1.; 6 Nov. 2015; M. Lukić leg.; CBSS (Ar 3780) 1 ô; same locality as for preceding; 6 Nov. 2015; A. Kirin leg.; CBSS (Ar 3776)

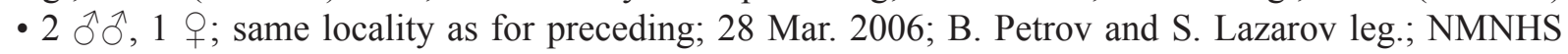

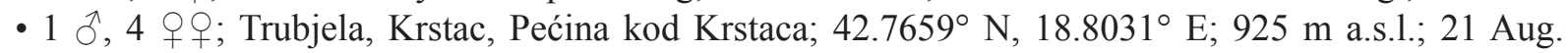
2017; M. Pavlek leg.; CBSS (Ar 5355) 1 đ; same collection data as for preceding; CBSS (Ar 5356) - 2 đิ (2 vials); Trubjela, Kita, pećina Ledenica na Kiti; $42.7729^{\circ}$ N, $18.7843^{\circ}$ E; 6 Aug. 1969 and 24 Jul. 1970; C. Deeleman leg.; NMNL (ARA 19033-34) • 1 đ̂, 2 우, 3 juvs; Podkita, Sirbaba pećina; 42.786 ${ }^{\circ}$ N, $18.825^{\circ}$ E; 940 m a.s.1.; 28 Mar. 2006; B. Petrov and S. Lazarov leg.; NMNHS • 1 ô, 1 , 
1 juv.; Vidrovan, Vidrovanska pećina; $42.858^{\circ} \mathrm{N}, 18.937^{\circ} \mathrm{E}$; $660 \mathrm{~m}$ a.s.1.; 29 Apr. 2016; T. Delić, D.

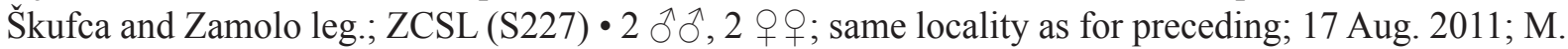

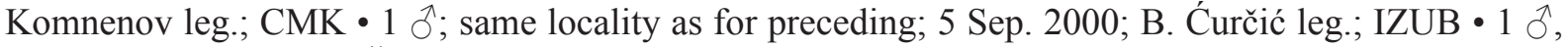
1 q; Golija Mt., Donje Čarađe, Drljača pećina; $43.023^{\circ}$ N, 18.674 E; 1035 m a.s.l.; 13 Aug. 2010; M.
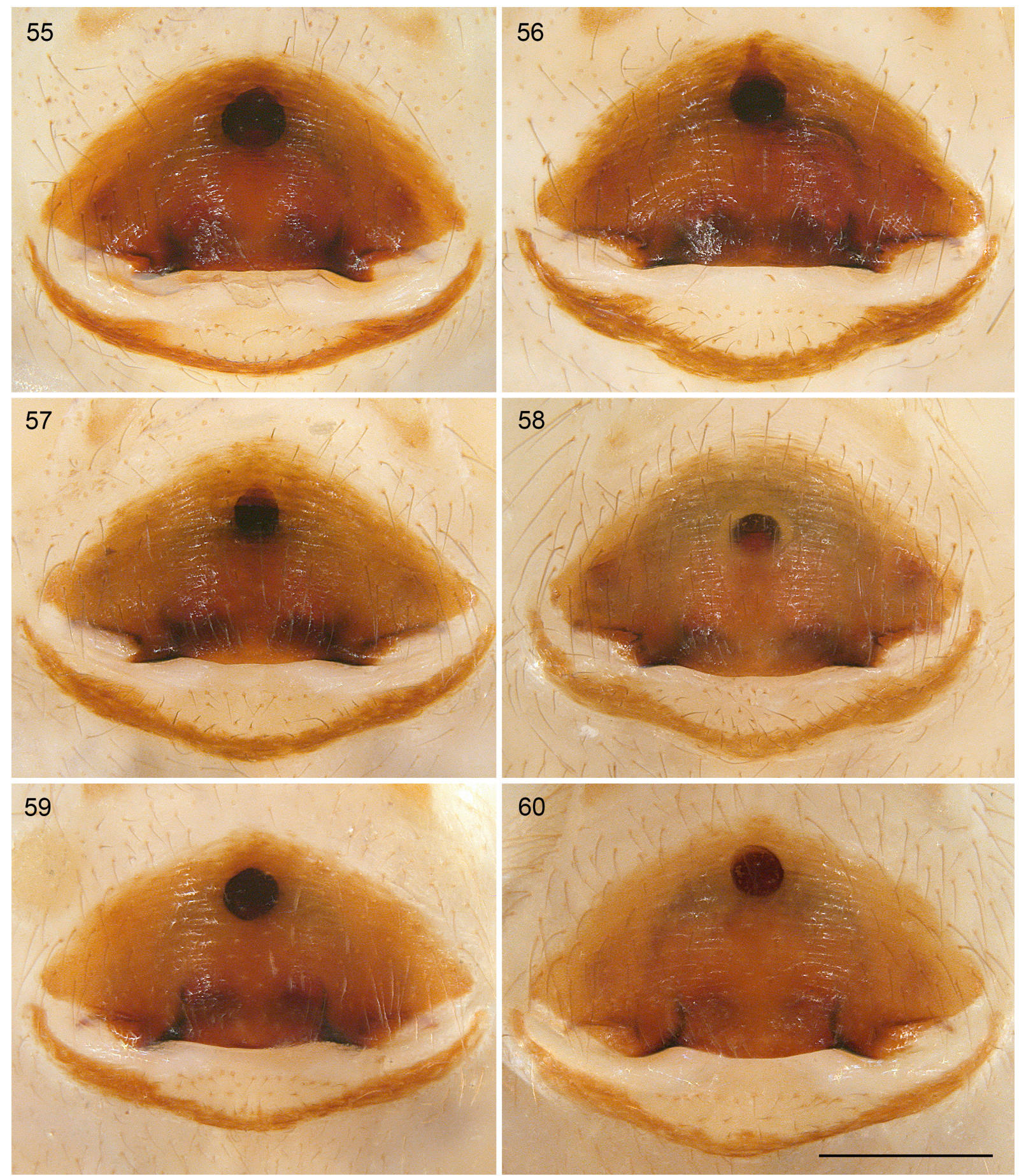

Figs 55-60. Stygopholcus skotophilus Kratochvíl, 1940; epigyna, ventral views. 55-56. Pećina u Ivici, Montenegro (ZFMK (Ar 22196)). 57. Ilijina pećina, Bosnia and Herzegovina (ZFMK (Ar 22198)). 58. Studena pećina, Bosnia and Herzegovina (CMK). 59-60. Specimens assigned tentatively from Grabova pećina, Sedlari, Bosnia and Herzegovina (MHNG). Scale bar: $0.5 \mathrm{~mm}$ (all at same scale). 

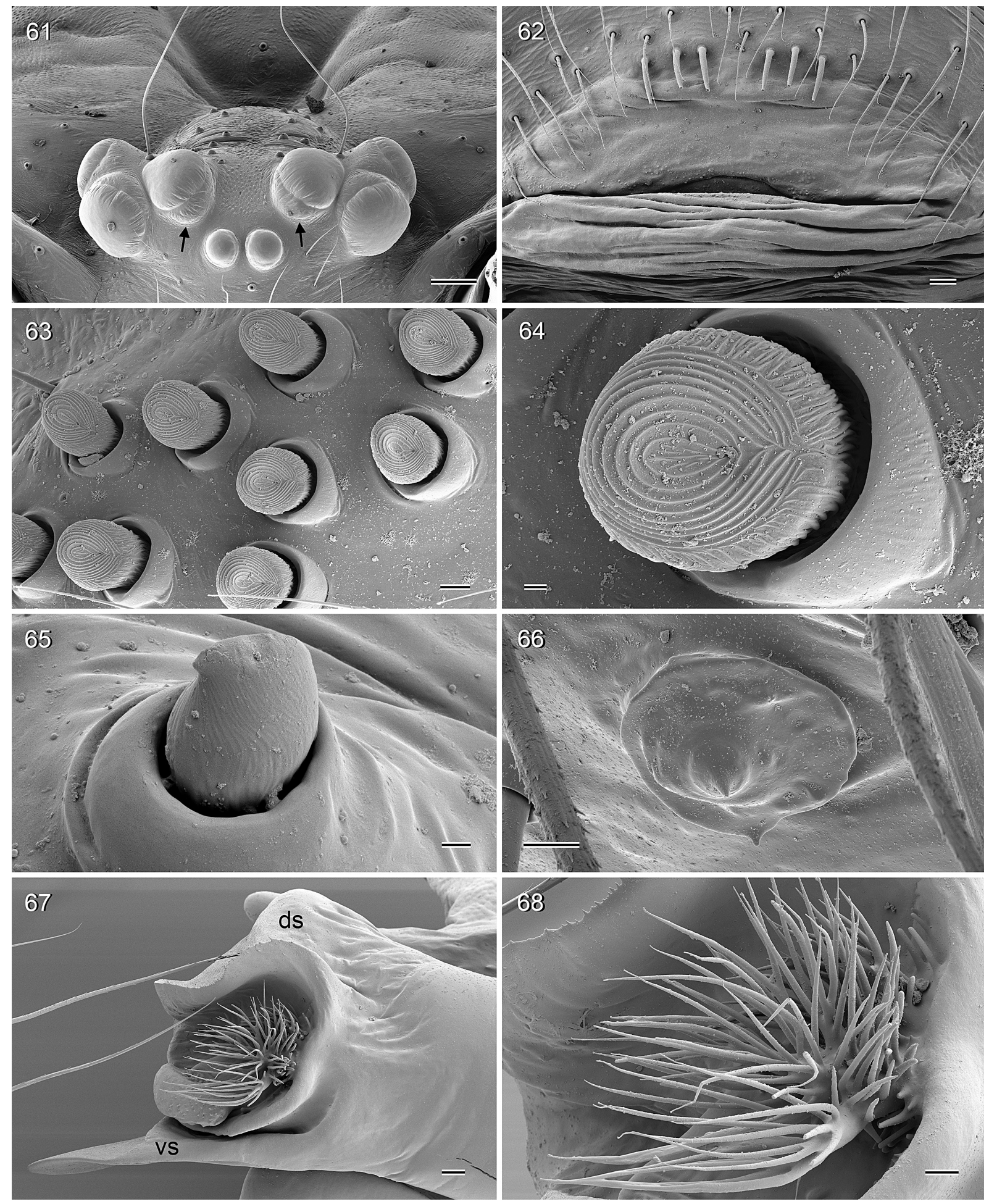

Figs 61-68. Stygopholcus skotophilus Kratochvíl, 1940; male and female from Ilijina pećina, Bosnia and Herzegovina (ZFMK (Ar 22198)). 61. Male ocular area, frontal view; arrows point at 'pseudoeyes'. 62. Male gonopore and epiandrous spigots. 63-64. Modified hairs frontally on male chelicerae. 65. Stridulatory pick on male palpal femur. 66. Male palpal tarsal organ. 67. Tip of left procursus, retrolateral view. 68. Hair-like structures on tip of procursus (detail of previous image). Abbreviations: $\mathrm{ds}=$ dorsal sclerite; $\mathrm{vs}=$ ventral sclerite. Scale bars: $61=100 \mu \mathrm{m} ; 62,67=20 \mu \mathrm{m} ; 63,66,68=10 \mu \mathrm{m}$; $64-65=2 \mu \mathrm{m}$. 

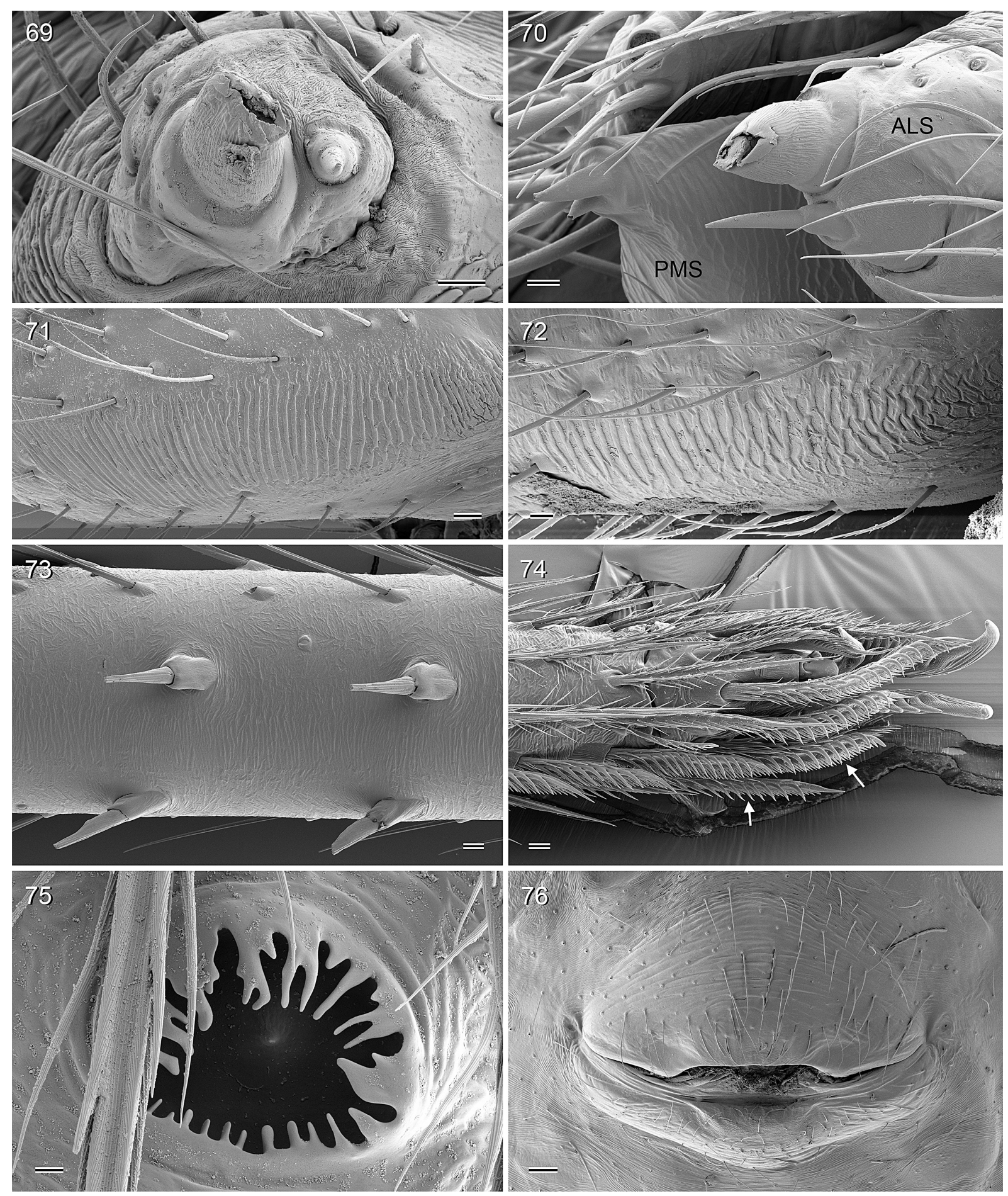

Figs 69-76. Stygopholcus skotophilus Kratochvíl, 1940; male and female from Ilijina pećina, Bosnia and Herzegovina (ZFMK (Ar 22198)). 69. Male ALS. 70. Female ALS and PMS. 71-72. Stridulatory files on male and female chelicerae. 73. Spines on male femur 1. 74. Tip of male right tarsus 4, ventral view; arrows point at comb-hairs. 75. Tarsal organ on male tarsus 4. 76. Epigynum, ventral view. Abbreviations: ALS = anterior lateral spinneret; PMS = posterior median spinneret. Scale bars: 69-70, $72,74=10 \mu \mathrm{m} ; 71,73=20 \mu \mathrm{m} ; 75=2 \mu \mathrm{m} ; 76=100 \mu \mathrm{m}$. 
Komnenov leg.; CMK・1 q; [near Javljem], Kerovačka pećina; $42.9981^{\circ}$ N, $18.7273^{\circ}$ E; 4 Aug. 1969; C. Deeleman leg.; NMNL (ARA 19036) • 1 đo, 1 क; Golija Mt., Stozi, Stoška pećina; $42.952^{\circ}$ N, $18.781^{\circ}$ E; 1230 m a.s.l.; 10 Aug. 2010; M. Komnenov leg.; CMK • 1 §ో; Golija Mt., Jasikovice, Lazareva pećina; 43.038 N, $18.699^{\circ}$ E; 8 Aug. 2010; M. Komnenov leg.; CMK. - Plužine • 1 đ̊; Zaborije, (Golija) Donja Brezna, Đokova Ledenica pećina; 43.0333 N, 18.8528 E; 1 Aug. 1972; C. Deeleman leg.; NMNL (ARA 19037).

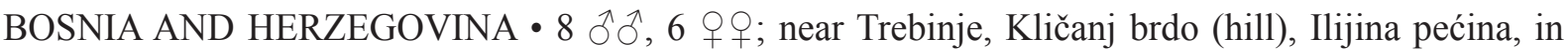
cave near eastern entrance; $42.7188^{\circ} \mathrm{N}, 18.3303^{\circ} \mathrm{E} ; 550 \mathrm{~m}$ a.s.1.; 26 May 2014; B.A. Huber and M. Komnenov leg.; ZFMK (Ar 22198) • 2 우, 2 juvs, in pure ethanol; same collection data as for preceding;

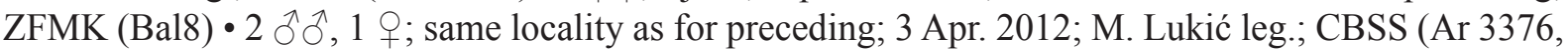
3379) 1 q, 1 juv.; Trebinje, Kličanj brdo, Vučja pećina; $42.716^{\circ} \mathrm{N}, 18.326^{\circ} \mathrm{E} ; 425 \mathrm{~m}$ a.s.1.; 27 Jan.

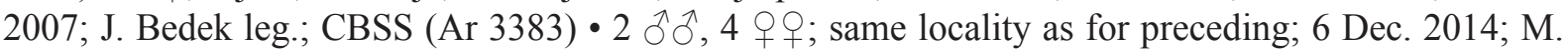
Pavlek leg.; CBSS (Ar 3481) • 2 juvs; same locality as for preceding; 27 Jan. 2007; H. Bilandžija leg.;

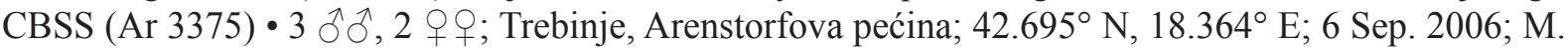
Komnenov leg.; CMK $\bullet 1$ § , 1 o; Trebinje, Petrina, Matulićeva pećina; $42.694^{\circ} \mathrm{N}, 18.362^{\circ} \mathrm{E}$; 6 Sep. 2006; M. Komnenov leg.; CMK • 2 đô, 1 क; Trebinje, Zupci, Vilina pećina; $42.663^{\circ} \mathrm{N}, 18.364^{\circ} \mathrm{E} ; 5$ Sep. 2006; M. Komnenov leg.; CMK・1 đ, 2 우, 21 juvs (2 vials); same locality as for preceding;

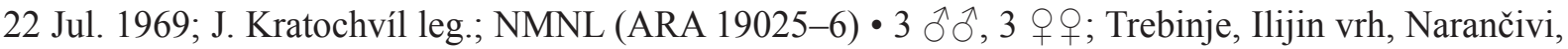

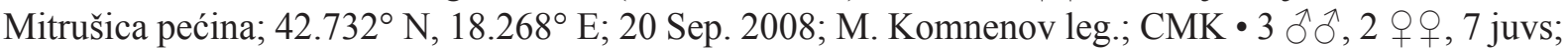
Trebinje, Dražin Do, Pećina kod Rupe ["pećina kod rupa, selo Dražin”]; $42.71^{\circ} \mathrm{N}, 18.30^{\circ}$ E; 24 Jul. 1969; J. Kratochvíl leg.; NMNL (ARA 19029) • 1 +, 3 juvs; Trebinje, Dražin do, Tučevac, in cave; 42.7142 ${ }^{\circ}$ N, $18.3033^{\circ}$ E; 21 Aug. 2017; A. Kirin leg.; CBSS (Ar 5372) • 1 q, Trebinje, Taleža, Taleža pećina; $42.714^{\circ} \mathrm{N}, 18.231^{\circ}$ E; $270 \mathrm{~m}$ a.s.1.; 7 Sep. 2006; M. Komnenov leg.; CMK 1 ô, 4 qo; near Trebinje, Bihovo, Pavlova pećina [= Ilijina pećina near Bihovo; cf. Lukić et al. 2020], in cave near entrance; $42.6665^{\circ} \mathrm{N}, 18.3078^{\circ} \mathrm{E}$; 420 m a.s.1.; 26 May 2014; B.A. Huber and M. Komnenov leg.; ZFMK (Ar 22199-22200) • 1 q, 2 juvs, in pure ethanol; same collection data as for preceding; ZFMK (Ba19) • 1 क , misidentified paralectotype of S. absoloni; same locality as for preceding; 20 Jul. 1912; K. Absolon leg.; MIZW • 1 q, 3 juvs; same locality as for preceding ("Eliashöhle"); date unknown; Verhoeff leg.; ZMB (9067) 1 q [and 1 male palp, possibly from different locality, according to label by P. Brignoli]; same collection data as for preceding; MCVR • 1 O, 1 \%; Zupci, Lemov do, Lemova pećina; $42.609^{\circ} \mathrm{N}, 18.399^{\circ}$ E; 14 Sep. 2006; M. Komnenov leg.; CMK・ 1 đ; Orjen, Ubla, Čavčina jama; $42.598^{\circ} \mathrm{N}, 18.520^{\circ}$ E; 18 Aug. 2007; M. Komnenov leg.; CMK • 1 O; Grebci, Debelin pećina ["Debeljin pećina, Osojnik"]; $42.7202^{\circ}$ N, 18.0955 E; 420 m a.s.1.; 16 Jul. 1972; J. Kratochvíl leg.; NMNL (ARA 19023) • 1 o, 1 juv.; Grebci, Kali pećina; $42.7406^{\circ} \mathrm{N}, 18.0626^{\circ}$ E; $525 \mathrm{~m}$ a.s.1.; 17 Jul. 1972; C. Deeleman leg.; NMNL (ARA 19024) • 1 क; Njemanica, unnamed cave below Jametina

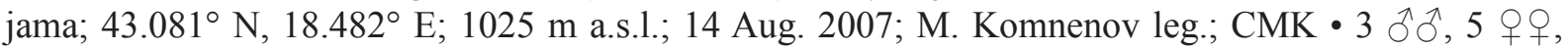
10 juvs (2 vials); Gacko, Korita, Pećina u Pejevim Torinama; 43.055 N, $18.493^{\circ}$ E; 11 Aug. 1968; C. Deeleman leg.; NMNL (ARA 19027-8) • 1 Oे, 1 क; Gacko, Dramešina, Studena pećina; $43.213^{\circ} \mathrm{N}$, $18.609^{\circ}$ E; 15 Aug. 2007; M. Komnenov leg.; CMK • 2 đ̊ đ; Gacko, Lukovice, Pećina u Lukovicama;

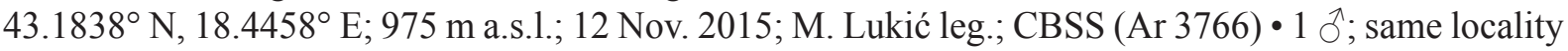
as for preceding; 12 Nov. 2015; A. Kirin leg.; CBSS (Ar 3764) • 1 ô, 1 क ; same collection data as for preceding; CBSS (Ar 3799).

CROATIA - Dubrovnik-Neretva • 1 ð̊; Konavle, Sniježnica, "Jama V=13"; $42.574^{\circ} \mathrm{N}, 18.352^{\circ} \mathrm{E}$;

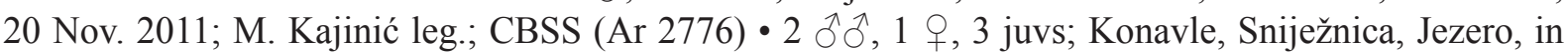

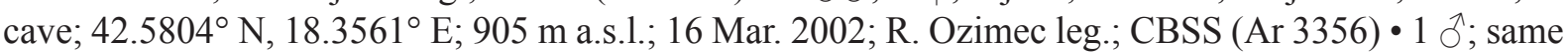
locality as for preceding; 1 Nov. 2016; A. Kirin leg.; CBSS (Ar 5255) 1 q, 5 juvs; same locality as for preceding; 1 Nov. 2016; T. Rožman leg.; CBSS (Ar 4776) • 2 juvs; same locality as for preceding; 28 May 2004; M. Pavlek leg.; CBSS (Ar 3360) • 1 ð̊; Konavle, Sniježnica, Škrabuljica, in cave; $42.5670^{\circ}$ N, 


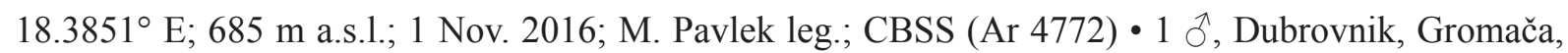
Špilja za Gromačkom vlakom; 42.7435 ${ }^{\circ}$, $18.0274^{\circ}$ E; 605 m a.s.1.; 22 Feb. 2019; A. Kirin leg.; CBSS (Ar 6819) • 1 क; same locality as for preceding; 11 Dec. 2019; M. Pavlek leg.; CBSS (Ar 6833) • 1 万; Konavle, Gruda, Vilina špilja; 42.5066 ${ }^{\circ}$ N, $18.3913^{\circ}$ E; 205 m a.s.l.; 6 Apr. 2010; R. Ozimec and A. Schönhofer leg.; ROC (R260).

\section{Localities unknown or unspecified}

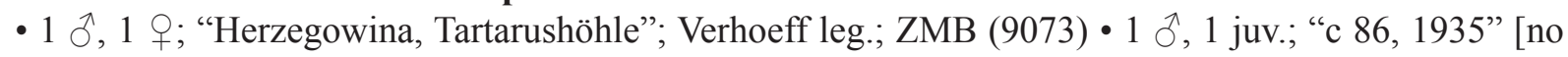
further data]; NMP• 1 juv.; "Wolfshöhle, Südherzegowina", "Vukovo jama bei Vrbanja"; Verhoeff leg.; ZMB (9069).

Credible published records (not examined or juveniles only) MONTENEGRO - Kotor • ${ }^{\top} O\left(\right.$ (?), material apparently lost; near Zvečava, Lopata pećina; $42.537^{\circ} \mathrm{N}$, $18.622^{\circ} \mathrm{E}$ [Naumova et al. 2019: $42.569^{\circ} \mathrm{N}, 18.662^{\circ} \mathrm{E}$ ]; 1932(?); J. Kratochvíl leg.(?) [Kratochvíl 1940] - 1 juv.; $2 \mathrm{~km} \mathrm{~W}$ Čora pećina, Matjaševica pećina; $42.595^{\circ} \mathrm{N}, 18.700^{\circ} \mathrm{E}$ [Naumova et al. 2019: $42.569^{\circ} \mathrm{N}$, $\left.18.662^{\circ} \mathrm{E}\right]$; 1932(?); J. Kratochvíl leg.(?) [Kratochvíl 1940]; NMP • ○̊ $\bigcirc(?)$, material apparently lost; Dragaljsko polje, near Dvoriško ždrijelo ("Dvorečko zdrielo"), Tomova pećina; $42.603^{\circ} \mathrm{N}, 18.675^{\circ} \mathrm{E}$;

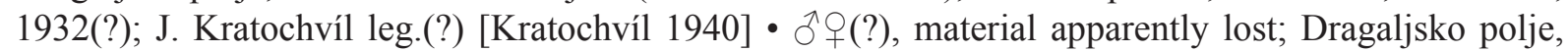
above Crni Nugli, Lakičević pećina; $42.597^{\circ} \mathrm{N}, 18.700^{\circ} \mathrm{E}$ [Naumova et al. $2019: 42.263^{\circ} \mathrm{N}, 19.033^{\circ} \mathrm{E}$ ]; 1932(?); J. Kratochvíl leg.(?) [Kratochvíl 1940] • đ̊(?), material apparently lost; 1 km above Lakičević pećina, Čra pećina; $42.594^{\circ} \mathrm{N}, 18.712^{\circ} \mathrm{E}$ [Naumova et al. 2019: $42.586^{\circ} \mathrm{N}, 18.697^{\circ} \mathrm{E}$ ]; 1932(?); J. Kratochvíl leg.(?) [Kratochvíl 1940] • đ̊(?), material apparently lost; near Jankovo selo, Pećina

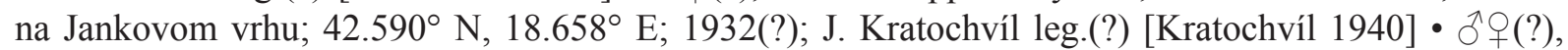
material apparently lost; $4 \mathrm{~km} \mathrm{~N}$ of Pećina na Jankovom vrhu, Pećina za Jankovim vrhom; $42.620^{\circ} \mathrm{N}$,

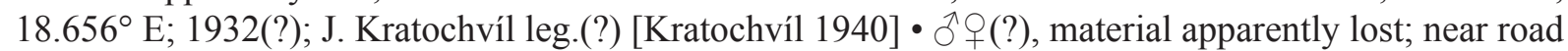
Crkvice-Veli vrh, cave near Crkvice; $42.560^{\circ} \mathrm{N}, 18.630^{\circ}$ E; 1932(?); J. Kratochvíl leg.(?) [Kratochvíl 1940] - $\widehat{O}$ ㅇ(?), material apparently lost; near Crkvice, $1 \mathrm{~km} \mathrm{~W}$ of Blagojević, Pećina kod Blagojevića; $42.573^{\circ} \mathrm{N}, 18.620^{\circ} \mathrm{E} ; 1932(?)$, J. Kratochvíl leg.(?) [Kratochvíl 1940] • đo $q(?)$, material apparently lost; near Poljana, Pećina kod Šunjevca; $42.577^{\circ}$ N, $18.648^{\circ}$ E; 1932(?); J. Kratochvíl leg.(?) [Kratochvíl 1940] - ${ }^{\top} O(?)$, material apparently lost; near Knezlac, Izeta pećina; $42.544^{\circ} \mathrm{N}, 18.689^{\circ} \mathrm{E}$ [Naumova

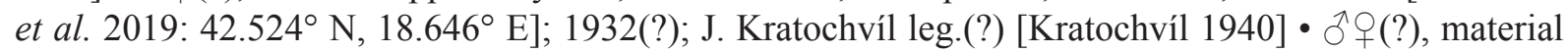
apparently lost; near Knezlac, near Izeta pećina, Studena pećina; $42.544^{\circ} \mathrm{N}, 18.689^{\circ} \mathrm{E}$; $1932($ )); J. Kratochvíl leg.(?) [Kratochvíl 1940] • đ̃o(?), material apparently lost; Pokljuka donja [Kratochvíl 1935: “dolnja”]; 4 km SE Knezlac; $42.542^{\circ}$ N, $18.693^{\circ}$ E; 1932(?); J. Kratochvíl leg.(?) [Kratochvíl 1940] • OP (?), material apparently lost; near Napode, near Risan-Crkvice road, Vilina ("Vilna") pećina; $42.5546^{\circ} \mathrm{N}, 18.6624^{\circ} \mathrm{E}$; 1932(?); J. Kratochvíl leg.(?) [Kratochvíl 1940] • $゚$ O(?), material apparently lost; near Vilina pećina, Pecinis kod Napode; $42.555^{\circ} \mathrm{N}, 18.662^{\circ} \mathrm{E}$ [Naumova et al. 2019: $42.519^{\circ} \mathrm{N}$, $\left.18.716^{\circ} \mathrm{E}\right] ; 1$ 1932(?); J. Kratochvíl leg.(?) [Kratochvíl 1940] • đ̊(?), material apparently lost; $1 \mathrm{~km}$ SW Risan, Golodražnica pećina; $42.503^{\circ} \mathrm{N}, 18.699^{\circ} \mathrm{E}$ [Naumova et al. $2019: 42.535^{\circ} \mathrm{N}, 18.689^{\circ} \mathrm{E}$; 185 m a.s.l.; 1932(?); J. Kratochvíl leg.(?) [Kratochvíl 1940]. - Nikšič • $゚$ O(?), material apparently lost; near Jabukovac, Leskova pećina; $42.83^{\circ}$ N, $18.47^{\circ}$ E; 1932(?); J. Kratochvíl leg.(?) [Kratochvíl 1940] - $\bigcirc(?)$, material apparently lost; near Leskova pećina, Golubnjačka pećina; $42.83^{\circ} \mathrm{N}, 18,47^{\circ} \mathrm{E}$

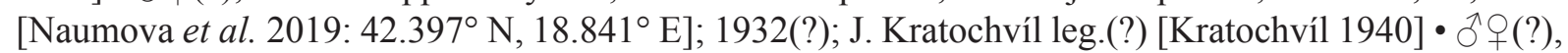
material apparently lost; near Grahovac, on Brdo Omutič mountain, Vodena pećina; $42.687^{\circ} \mathrm{N}, 18.617^{\circ} \mathrm{E}$; 1932(?); J. Kratochvíl leg.(?) [Kratochvíl 1940] • đ̃o(?), material apparently lost; above Osječenica, Bobjerska pećina; $42.699^{\circ} \mathrm{N}, 18.634^{\circ} \mathrm{E}$ [Naumova et al. 2019: 42.674 $\left.\mathrm{N}, 18.662^{\circ} \mathrm{E}\right]$; $1932(?)$; J. Kratochvíl leg.(?) [Kratochvíl 1940] • đo (?), material apparently lost, Elazov dô, near Zagora, Elazova pećina; $42.704^{\circ} \mathrm{N}, 18.655^{\circ} \mathrm{E}$ [Naumova et al. 2019: 42.706 $\left.{ }^{\circ} \mathrm{N}, 18.673^{\circ} \mathrm{E}\right]$; 1932(?); J. Kratochvíl leg. (?) [Kratochvíl 1940] • $\bigcirc(?)$, material apparently lost; Troškarica planina mountains; Vranova jama; $42.838^{\circ} \mathrm{N}, 18.551^{\circ} \mathrm{E} ; 1932(?) ; \mathrm{J}$. Kratochvíl leg.(?) [Kratochvíl 1940]. 
Possible record (not examined)

The following record in Kratochvíl (1940) is dubious because he assigned his specimen(s) to S. absoloni, but specimens from the closest caves we examined (Debelin pećina, Kali pećina, Špilja za Gromačkom vlakom) were all unambiguous $S$. skotophilus. We thus assume that Kratochvíl misidentified his specimen(s) from this cave.

BOSNIA AND HERZEGOVINA • ^q(?), material apparently lost; near Grebci, Grabovica pećina; $42.733^{\circ} \mathrm{N}, 18.081^{\circ} \mathrm{E} ; 1932(?) ; \mathrm{J}$. Kratochvíl leg.(?) [Kratochvíl 1940].

\section{Material assigned tentatively}

The material listed here originates from caves around Sedlari in Bosnia and Herzegovina. This material has been studied before by Kratochvíl (1940) and Senglet (2001), and both authors assigned most of the specimens to $S$. skotophilus (only the female from Medja pećina was assigned to S. absoloni by Kratochvíl 1940). However, both males and females from these caves show some small but consistent differences to $S$. skotophilus (see section on variation below) so the material is here listed apart to draw attention to this potential separate species (of which no fresh material is available).

BOSNIA AND HERZEGOVINA • 2 $\widehat{\jmath}, 13$ $\rightarrow$ , 3 juvs ( 2 vials); "Sedlari/Popovo-Polje" [according to Senglet 2001 the specimens were collected in Grabova pećina]; $42.825^{\circ} \mathrm{N}, 18.055^{\circ} \mathrm{E}$; $18 \mathrm{Sep} .1970$; A. Senglet leg.; MHNG • 2 juvs; same locality as for preceding ("Grabova peć kod Sedlari”); 17 Jul.

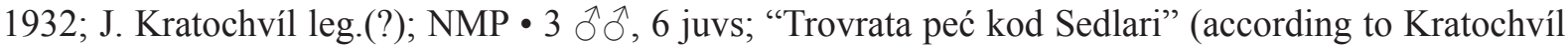
1940, this cave is near Grabova pećina); 17 Jul. 1932; J. Kratochvíl leg.(?); NMP • 6 juvs; "Vodena peć kod Sedlari" [according to Kratochvíl 1940, this cave is near Trovrata pećina and Grabova pećina]; 17 Jul. 1932; J. Kratochvíl leg.(?); NMP • 1 +, 2 juvs; “Medja peć na Ilijina brdu, Grmljani” [according to Kratochvíl 1940 this cave is located $2 \mathrm{~km} \mathrm{~W}$ of Grmljani]; 42.84 N, 18.01 W; 1932(?); J. Kratochvíl leg.(?); NMP.

\section{Redescription}

Male (type locality; ZFMK (Ar 22196))

MEASUREMENTS. Total body length 5.5, carapace width 2.2. Distance PME-PME $160 \mu \mathrm{m}$; diameter PME $150 \times 180 \mu \mathrm{m}$; distance PME-ALE $20 \mu \mathrm{m}$; diameter AME $65 \mu \mathrm{m}$; distance AME-AME $30 \mu \mathrm{m}$. Leg 1: $51.2(13.7+0.9+13.6+20.3+3.2)$, tibia $2: 10.0$, tibia $3: 8.2$, tibia $4: 8.9$; tibia $1 \mathrm{~L} / \mathrm{d}: 62$.

COLOR (in ethanol). Carapace pale whitish to ochre-yellow, posterior half, ocular area, and clypeus slightly darker (light brown); sternum light brown, with dark brown margins and three pairs of dark triangular radial marks; legs pale ochre-yellow to light brown, without dark rings, without dark lines; abdomen pale gray, with distinct dark dorsal marks in posterior third and lateral marks in posterior half; ventrally with light brown genital area, indistinct diffuse purplish marks in front and behind genital area and in front of spinnerets.

Body. Habitus as in Fig. 2. Ocular area slightly raised. Carapace with deep median pit (Fig. 61) and pair of very shallow indistinct furrows diverging from pit to posterior margin of carapace. Clypeus unmodified. Sternum wider than long (1.50/1.05), unmodified. Abdomen oval, pointed at spinnerets; gonopore with six epiandrous spigots (Fig. 62); ALS with two spigots each (Fig. 69).

Chelicerae. As in Figs 51-52, with pair of large frontal apophyses set with 2 modified hairs each, with 12 and 13 modified hairs each on frontal face (Figs 63-64); with stridulatory ridges (Fig. 71).

PALPS. As in Figs 6-8; coxa with low retrolateral hump; trochanter barely modified; femur with distinct retrolateral process proximally, with stridulatory pick (modified hair) proximally on prolateral side (Fig. 65); femur-patella joints shifted towards prolateral (arrows in Fig. 6); tibia very large, with two 
trichobothria; tibia-tarsus joints shifted towards retrolateral (arrows in Fig. 8); tarsus with exposed tarsal organ (Fig. 66); procursus dorsally with curved hairs (distal third curved 'upwards'), with strong hump on prolateral side; procursus tip with projecting dorsal sclerite, strong hinged ventral sclerite, and membranous central part with brush of hair-like processes on retrolateral side (Figs 67-68), with weakly sclerotized cylindrical process on prolateral side bordering ventral sclerite (arrow in Fig. 45). Genital bulb (Figs 47-50) with basal sclerite connecting to tarsus, small transparent dorsal process, and main sclerite consisting of retrolateral process and dorsal process; retrolateral process with bifid tip, distal part on ventral side thickened and heavily sclerotized; dorsal process with two finger-shaped tips, ventral tip simple, retrolateral tip with additional strong protrusion on ventral side.

LEGS. With short spines ventrally on femur 1 in two rows (Fig. 73), prolateral row $\sim 23$ spines, retrolateral row $\sim 28$ spines; without curved hairs; few vertical hairs; retrolateral trichobothrium of tibia 1 at $4 \%$; prolateral trichobothrium absent on tibia 1, present on other tibiae; tarsus 1 with irregular platelets rather than with distinct pseudosegments.

Male (variation)

Males from most other localities paler, without lateral dark marks on abdomen, with fewer median dark marks (restricted to area above spinnerets), rarely without abdominal marks. Tibia $1(\mathrm{~N}=35): 11.1-14.7$ (mean 12.8); tibia $2(\mathrm{~N}=37$ ): 8.1-10.8 (mean 9.6); spines on femur 1: $32-58$ (mean 52); modified hairs on each cheliceral basis $(\mathrm{N}=74)$ : $9-16$ (mean 12.3); modified hairs on each cheliceral apophysis $(\mathrm{N}=74)$ : $1-5$ (mean 2.7); chelicerae maximum width $(\mathrm{N}=36)$ : 0.74-0.86 (mean 0.80$)$; procursus length $(\mathrm{N}=37)$ : $1.28-1.48$ (mean 1.39); bulbal measures A-E: A $(\mathrm{N}=20)$ : 0.28-0.31 (mean 0.30); $\mathrm{B}(\mathrm{N}=20)$ : 0.08-0.10 (mean 0.09); $\mathrm{C}(\mathrm{N}=20)$ : 0.13-0.15 (mean 0.14); $\mathrm{D}(\mathrm{N}=20)$ : 0.28-0.33 (mean 0.31); E $(\mathrm{N}=15)$ : $0.82-0.90$ (mean 0.87); epiandrous spigots $(\mathrm{N}=26)$ : 5-8 (mean 6.1).

Males from near Sedlari differ slightly in bulbal measure A smaller (0.25-0.28) and bulbal measure B smaller (0.07-0.08); in addition, the smaller wart on the retrolateral bulbal process (arrow in Fig. 48) is more strongly developed.

\section{Female}

In general similar to male, but femur 1 without spines, with pair of indistinct processes posteriorly on carapace and pair of opposing poorly visible plates on abdomen; distance between processes/plates: 1.1. Tibia $1(\mathrm{~N}=36)$ : $10.4-14.3$ (mean 12.4); tibia $2(\mathrm{~N}=36)$ : 7.2-10.3 (mean 9.0); epigynum width $(\mathrm{N}=36)$ : 1.02-1.46 (mean 1.21); epigynum length $(\mathrm{N}=36)$ : 0.54-0.68 (mean 0.61); epigynal measure $\mathrm{F}(\mathrm{N}=36)$ : $0.42-0.58$ (mean 0.47$)$. Epigynum anterior plate semicircular, weakly protruding, with weak transversal furrows (Figs 55-58, 76), with internal structures visible in uncleared specimens: round anterior structure, short and indistinct parallel sclerites posteriorly wide apart; posterior plate short but wide. Internal genitalia (Figs 53-54, 147-148) with distinctive ventral arc (Fig. 154; approximately as long as wide), with large pore plates (Fig. 54).

Females from near Sedlari differ in having the parallel sclerites posteriorly in the female internal genitalia closer together (epigynal measure F: 0.32-0.36; Figs 59-60) and slightly more distinct.

\section{Distribution}

Known from the southern border area separating Bosnia and Herzegovina from Montenegro, and from the southernmost tip of Croatia (Fig. 168). 
Stygopholcus montenegrinus Kratochvíl, 1940 orig. stat.

Figs 3, 77-112, 149-150, 155

Stygopholcus montenegrinus Kratochvíl, 1940: 20, fig. 8.

Stygopholcus skotophilus montenegrinus - Senglet 1971: 354, figs 42-43; 2001: 65. - Naumova et al. 2016: 434 (part; see S. skotophilus).

Stygopholcus photophilus (misidentification) - Deltshev et al. 2011: 130 (specimen from near Kozhnja only).

Hoplopholcus scotophilus (misidentification) -Tomić et al. 2000: 35P (specimens from near Velja cave). "S109 Stygopholcus absoloni? Bal11" - Eberle et al. 2018. — Huber et al. 2018.

\section{Diagnosis}

Distinguished from other species in the 'northern clade' (S. absoloni; S. skotophilus) by narrow, weakly sclerotized prolateral sclerite on procursus (arrows in Figs 77-78, 100); from S. skotophilus also by bulb measures A and C (Fig. 158; larger than in S. skotophilus, no overlap), by epigynal measure F (Fig. 164; smaller than in S. skotophilus, with little overlap); from S. absoloni also by straight procursus (rather than curved toward ventral; cf. Fig. 44), by longer procursus (Fig. 161; with little overlap), by shape of retrolateral bulbal process in retrolateral view (Fig. 79; large notch on ventral side), by bulb measures B and D (Figs 157, 159; larger than in S. absoloni, no overlap) and bulb measure E (Fig. 160; larger than S. absoloni, with little overlap).

\section{Type material}

\section{Holotype}

MONTENEGRO - Nikšič • + (without epigynum), near Nikšič, Studenačka pećina; $42.7680^{\circ} \mathrm{N}$, $18.9312^{\circ}$ E [Naumova et al. 2019: 42.826 N, 18.786 E]; 6 Sep. 1936; J. Kratochvíl leg.(?); NMP.

\section{Other material examined}

MONTENEGRO - Nikšič • 1 juv.; same collection data as for holotype; NMP • $9 \hat{\partial} \partial, 8$ q $q$; near Nikšić, Studenačka pećina, in cave near entrance and among rocks near cave; $42.7680^{\circ} \mathrm{N}, 18.9312^{\circ} \mathrm{E}$; 640 m a.s.1.; 27 May 2014; B.A. Huber and M. Komnenov leg.; ZFMK (Ar 22201) • 1 ô, 1 ค, 1 juv.

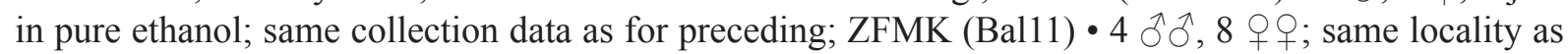
for preceding; 16 Sep. 1970; A. Senglet leg.; MHNG $\bullet 1$ गे, 1 \%; Trebjesa, Vojna pećina; $42.765^{\circ}$ N, $18.959^{\circ}$ E; 19 Aug. 2011; M. Komnenov leg.; CMK • 1 §o, 1 क; Velja pećina; $42.728^{\circ} \mathrm{N}, 18.943^{\circ} \mathrm{E}$; 16 Aug. 2011; M. Komnenov leg.; CMK $\bullet 1 \hat{\jmath}, 3$ 우, totally fragmented; unnamed cave above Velja pećina; $42.73^{\circ} \mathrm{N}, 18.94^{\circ}$ E; 6 Sep. 2000; B. Ćurčić leg.; IZUB • 2 + $q$; Carev Most, Budoška pećina; $42.7303^{\circ} \mathrm{N}, 18.9498^{\circ} \mathrm{E}$; 26 Jul. 1969; C. Deeleman leg.; NMNL (ARA 19035) • 2 ઊ̊, 2 우; Slivlje, Golubešnica pećina, 42.724 N, 19.013 ${ }^{\circ}$ E; 18 Aug. 2011; M. Komnenov leg.; CMK. - Podgorica

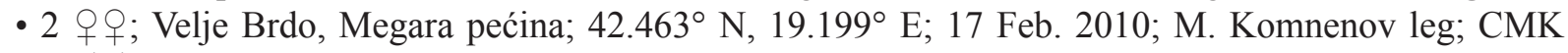

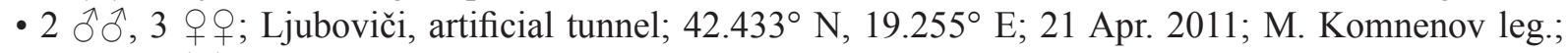

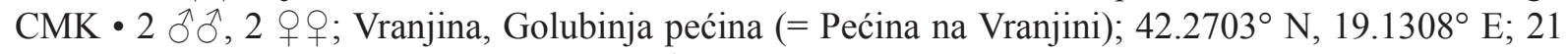
Apr. 2011; M. Komnenov leg.; CMK • 1 đ, 4 juvs; same locality as for preceding; 23 Aug. 2017; A. Kirin leg.; CBSS (Ar 5342) • 1 o, 1 juv.; same locality as for preceding; 23 Aug. 2017; M. Pavlek leg.; CBSS (Ar 5329) • 1 juv.; same locality as for preceding; 23 Aug. 2017; A. Kirin leg.; CBSS (Ar 5334) - 10 juvs; same locality as for preceding; 31 Jul. 1969; J. Kratochvíl leg.; NMNL (ARA 19041). - Cetinje

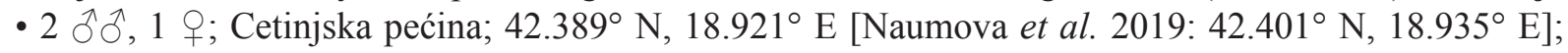
10 Aug. 2011; M. Komnenov leg.; CMK 11 ○ं; Lipska pećina; $42.375^{\circ} \mathrm{N}, 18.955^{\circ} \mathrm{E}$ [Naumova et al. 2019: $42.375^{\circ} \mathrm{N}, 19.952^{\circ}$ E]; 21 Apr. 2011; M. Komnenov leg.; CMK ・ 2 ๙ same locality as for preceding ("Lipa Dobersko"); 16 Sep. 1970; A. Senglet leg.; MHNG・1 ð’; Začir, 


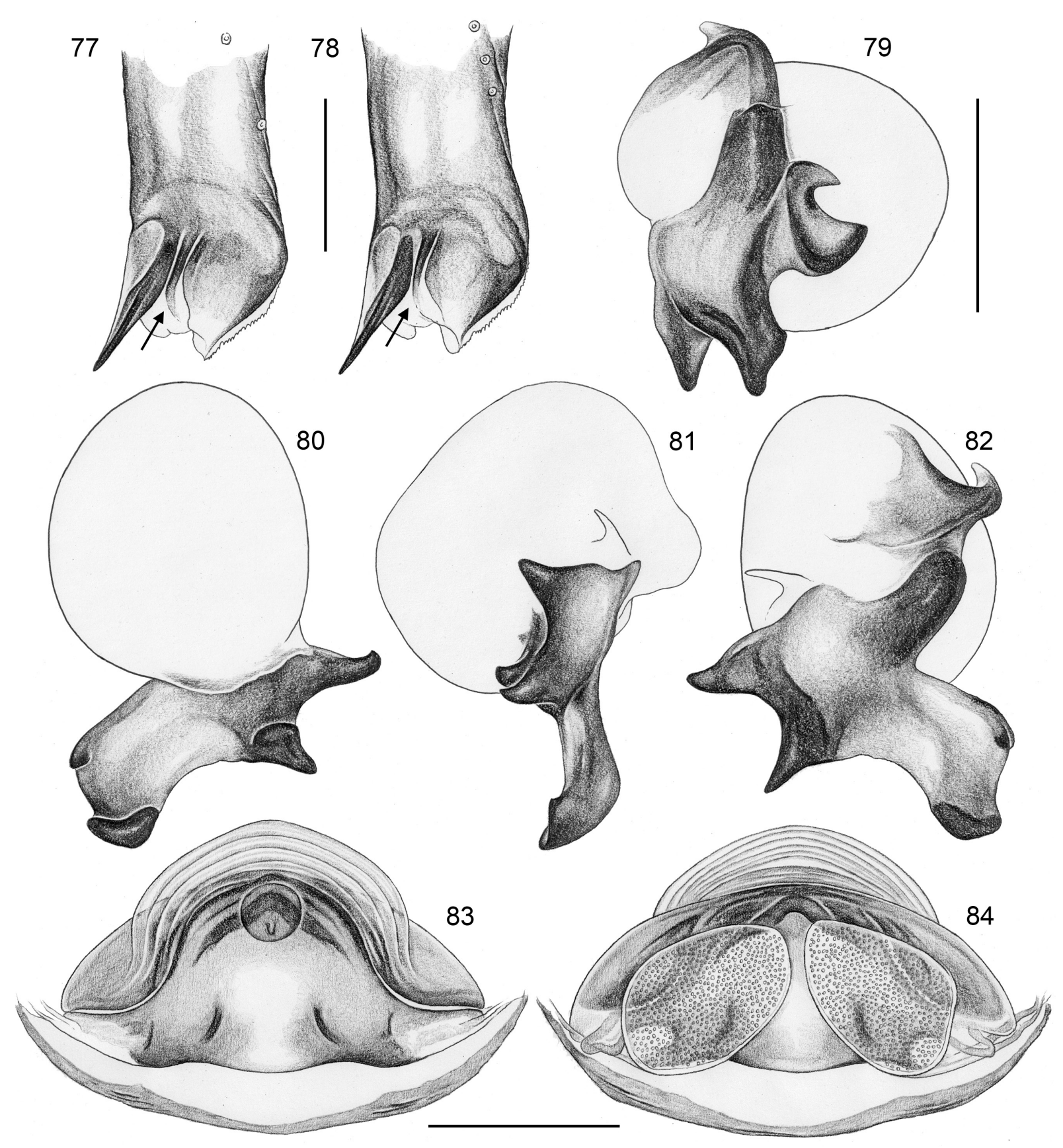

Figs 77-84. Stygopholcus montenegrinus Kratochvíl, 1940. 77-78. Males from Studenačka pećina, Montenegro (ZFMK (Ar 22201)) and from Zeze Cave, Albania (CMK), tips of left procursi, prolateral (slightly ventral) views; arrows point at distinctive prolateral sclerites. 79-82. Male from Studenačka pećina (ZFMK (Ar 22201)), left genital bulb, retrolateral, ventral, prolateral-distal, and dorso-distal views. 83-84. Female from Studenačka pećina (ZFMK (Ar 22201)), cleared female genitalia, ventral and dorsal views. Scale bars: $0.5 \mathrm{~mm}$. 
Začirska pećina; $42.343^{\circ}$ N, $18.992^{\circ}$ E; 5 Oct. 2010; M. Pavičević leg.; CMK • 1 ô; same locality as for preceding; 21 Aug. 2017; T. Rožman leg.; CBSS (Ar 5405) • 3 đ̂̃, 1 q; Rijeka Crnojevića, Obodska pećina; $42.352^{\circ}$ N, $19.005^{\circ}$ E; 19 Apr. 2011; M. Komnenov leg.; CMK • 1 q; same locality
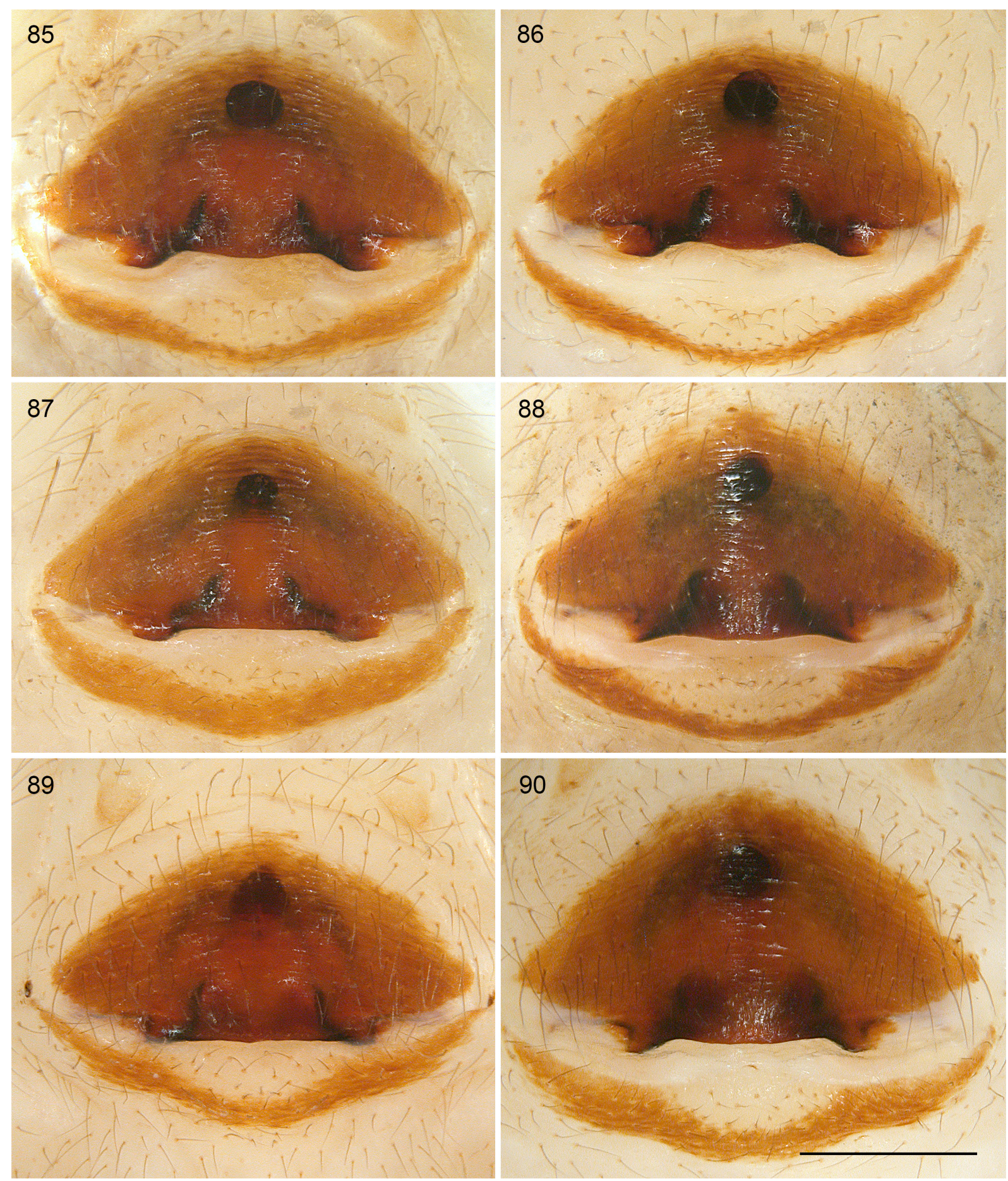

Figs 85-90. Stygopholcus montenegrinus Kratochvíl, 1940; epigyna, ventral views. 85-86. Studenačka pećina, Montenegro (ZFMK (Ar 22201)). 87. Golubinja pećina, Montenegro (CMK). 88. Ljuboviči, Montenegro (CMK). 89. Nëngurit Cave, Albania (CMK). 90. Petnjik Cave, Montenegro (CMK). Scale bar: $0.5 \mathrm{~mm}$ (all at same scale). 

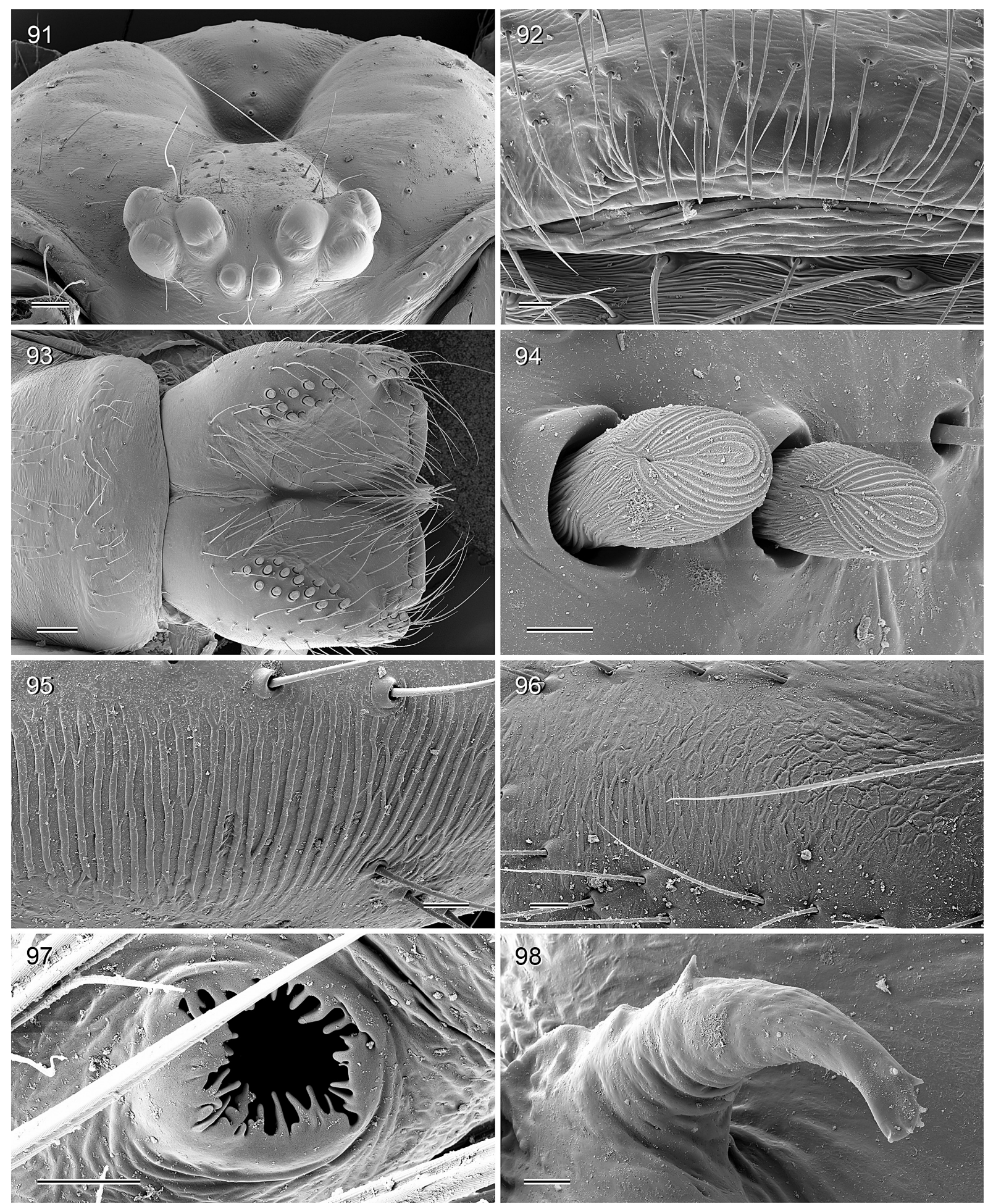

Figs 91-98. Stygopholcus montenegrinus Kratochvíl, 1940; male and female from Studenačka pećina, Montenegro (ZFMK (Ar 22201)). 91. Male carapace and ocular area, frontal-dorsal view. 92. Male gonopore and epiandrous spigots. 93. Male clypeus and chelicerae, frontal view. 94. Modified hairs frontally on male chelicerae. 95-96. Stridulatory files on male and female chelicerae. 97. Tarsal organ on male tarsus 1. 98. Transparent process on genital bulb. Scale bars: $91,93=100 \mu \mathrm{m} ; 92,95-96=20 \mu \mathrm{m}$; $94,97-98=10 \mu \mathrm{m}$. 

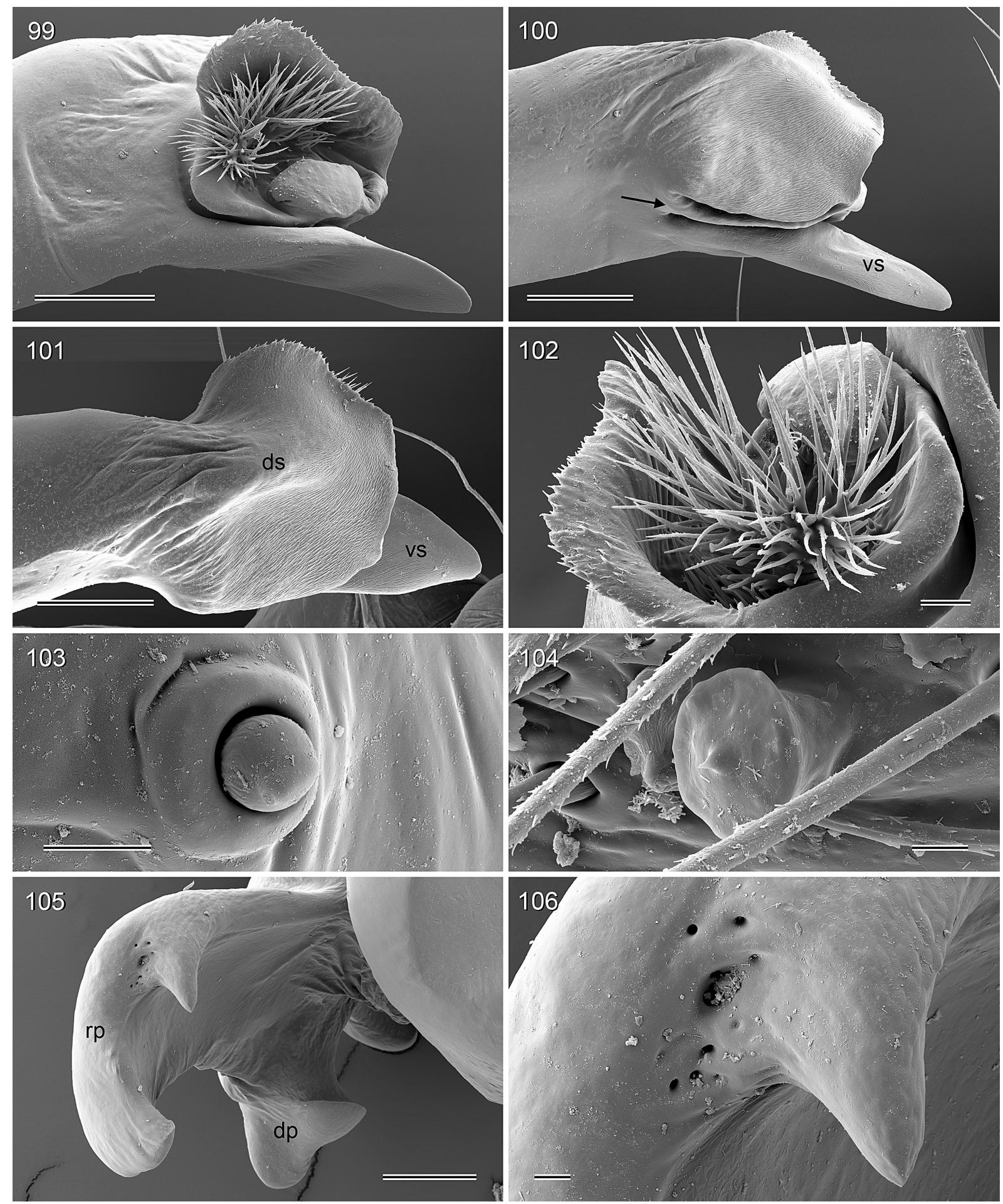

Figs 99-106. Stygopholcus montenegrinus Kratochvíl, 1940; male and female from Studenačka pećina, Montenegro (ZFMK (Ar 22201)). 99. Tip of right procursus, retrolateral view. 100-101. Tip of left procursus, prolateral and dorsal views; arrow points at distinctive prolateral sclerite. 102. Hair-like structures on tip of procursus. 103. Stridulatory pick on male palpal femur. 104. Male palpal tarsal organ. 105. Retrolateral bulbal process, distal view. 106. Possible glandular pores on retrolateral bulbal process (detail of previous photo). Abbreviations: $\mathrm{dp}=$ dorsal process; $\mathrm{ds}=$ dorsal sclerite; $\mathrm{rp}=$ retrolateral process; vs = ventral sclerite. Scale bars: 99-101, $105=100 \mu \mathrm{m} ; 102=20 \mu \mathrm{m} ; 103-104,106=10 \mu \mathrm{m}$. 
as for preceding; 23 Aug. 2017; M. Pavlek leg.; CBSS (Ar 5484) • 1 o, 4 juvs; same collection data as for preceding; A. Kirin leg.; CBSS (Ar 5485) • 3 $\widehat{\jmath}, 1$ q; Jankoviči Krš, Duruk pećina; $42.364^{\circ}$ N, $19.023^{\circ}$ E; 16 Apr. 2011; M. Komnenov leg.; CMK • 1 q; near river Rijeka Crnojevića, Žmrljevica pećina; $42.361^{\circ} \mathrm{N}, 19.050^{\circ}$ E; 16 Apr. 2011; M. Komnenov leg.; CMK・ 1 đ̊; Vrela, Pećina u Vrelima;

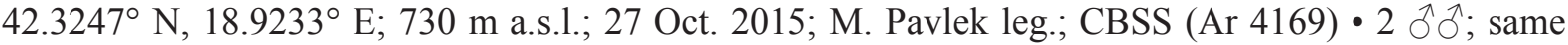
collection data as for preceding; CBSS (Ar 3669) • 2 స̂, 1 क; Trnovo, Babatuša pećina; $42.291^{\circ} \mathrm{N}$,

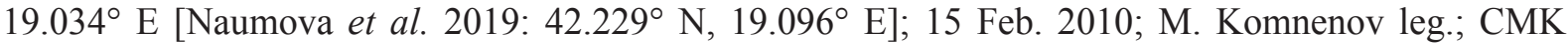
- 1 ภ (palps and chelicerae missing); same locality as for preceding, ("Bobotuša pećina"); 1932(?); J. Kratochvíl leg.(?); NMP・ 1 ○ૈ, 2 우; Trnovo, Grbočica pećina; $42.288^{\circ}$ N, $19.036^{\circ}$ E; 15 Apr. 2011;

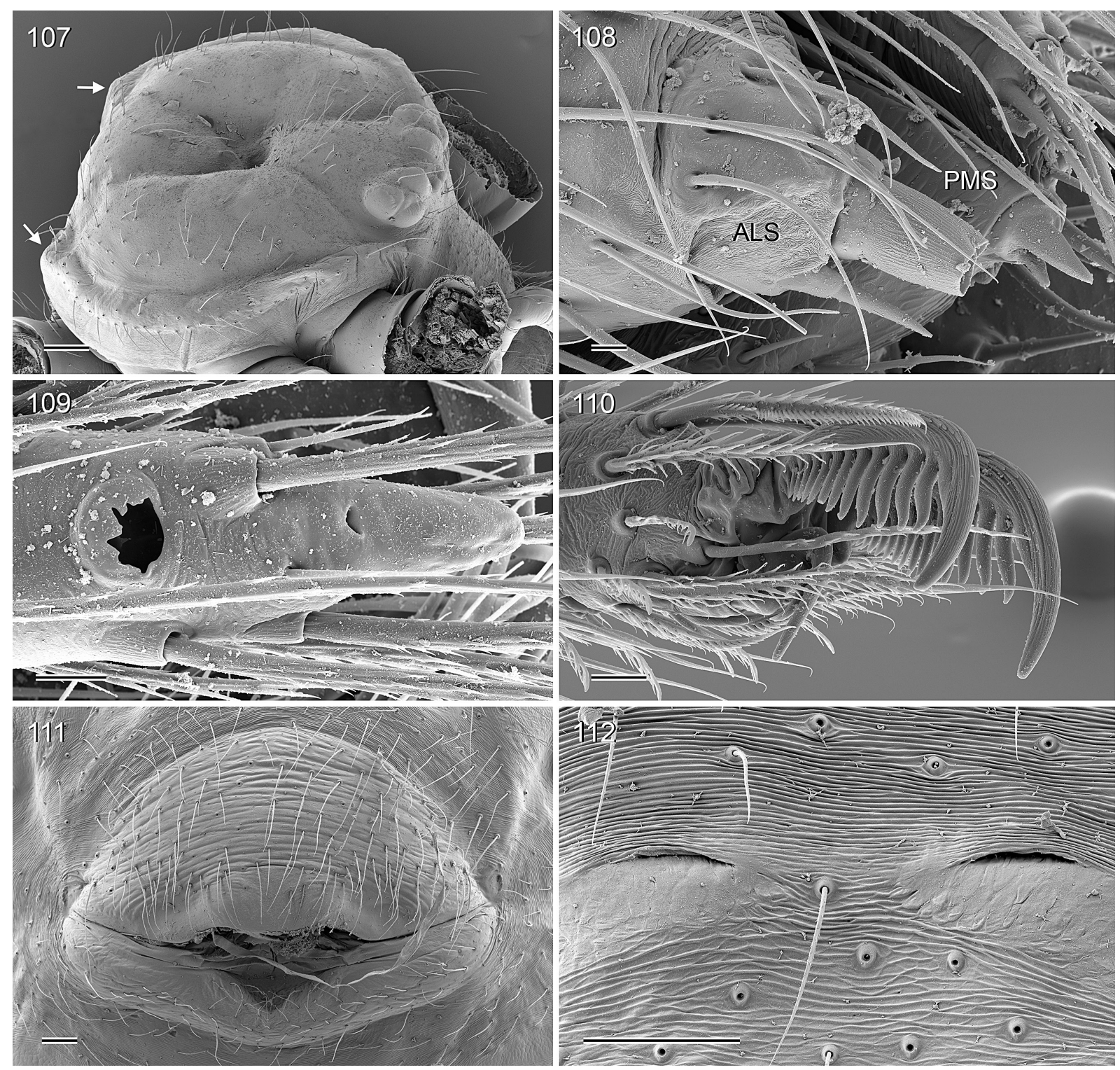

Figs 107-112. Stygopholcus montenegrinus Kratochvíl, 1940; male and female from Studenačka pećina, Montenegro (ZFMK (Ar 22201)). 107. Female prosoma; arrows point at humps opposing modified areas on abdomen. 108. Female anterior lateral spinneret (ALS) and posterior median spinneret (PMS). 109. Tip of female palp, dorsal view. 110. Tip of female tarsus 1. 111. Epigynum, ventral view. 112. Indentations (muscle attachment sites) between epigynum and spinnerets. Scale bars: $107=200 \mu \mathrm{m}$; $108=10 \mu \mathrm{m} ; 109-110=20 \mu \mathrm{m} ; 111-112=100 \mu \mathrm{m}$. 
M. Komnenov leg.; CMK 1 1 , 1 juv.; same locality as for preceding; 9 Nov. 2015; A. Kirin leg.;

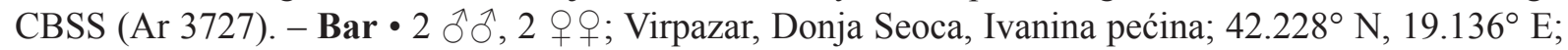
100 m a.s.1.; 3 Apr. 2007; M. Komnenov leg.; CMK • 1 \%; Seoca, Golubova pećina, hillside forest, under stones and sieving; $42.208^{\circ} \mathrm{N}, 19.131^{\circ} \mathrm{E} ; 440 \mathrm{~m}$ a.s.1.; 12 Aug. 2006; B. Petrov and S. Lazarov leg.; NMNHS. - Kolašin • 1 đ̃, 1 o; Monastery Morača; $42.7648^{\circ}$ N, $19.3931^{\circ}$ E; 300 m a.s.1.; 8 May 2006; A. Schönhofer leg.; SMF.

MONTENEGRO - unknown municipality $\bullet 3+q$ prosomata and $2 q q$ abdomens; "Južna Crna Gora, neka pećina" (= southern Montenegro, some cave); date unknown; P. Dabović leg.; NMP.

ALBANIA - Shkodra • 2 ふึે, 1 q; Galeria Iliriana, Tuneli i Gencit; $42.032^{\circ}$ N, $19.474^{\circ}$ E; 24 Nov. 2000; A. Zhalov leg.; NMNHS.

Credible published records (not examined)

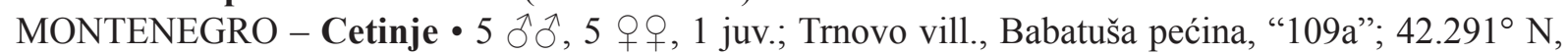
$19.034^{\circ}$ E; 24 Mar. 2006; S. Lazarov and B. Petrov leg. [Naumova et al. 2016] • 1 đ, 1 q, 1 juv., Cetinje town, unnamed cave above monastery, "102"; $42.39^{\circ}$ N, 18.92 E; 25 Mar. 2006; B. Petrov and S. Lazarov leg. [Naumova et al. 2016].

\section{Material assigned tentatively}

The material listed here originates from SE Montenegro (Podgorica) and NW Albania. It strongly resembles $S$. montenegrinus but shows some small though consistent differences (see section on variation below). The material is listed apart to draw attention to this potential separate species.

MONTENEGRO - Podgorica • 1 ô, 1 क; cave in Moraća canyon ["grotje langs de Moraća, $\sim 14 \mathrm{~km}$

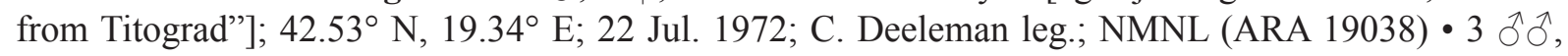
2 우, 4 juvs; Peuta, Dučica pećina; $42.4621^{\circ}$ N, $19.3307^{\circ}$ E; 19 Jul. 1972; C. Deeleman leg.; NMNL (ARA 19039) • 1 juv.; Peuta, Pećina od Zavora; $42.46^{\circ}$ N, $19.33^{\circ}$ E; 19 Jul. 1972; C. Deeleman leg.;

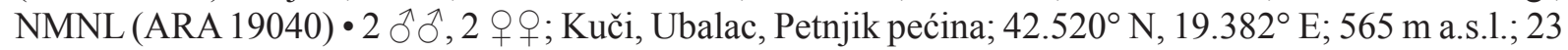

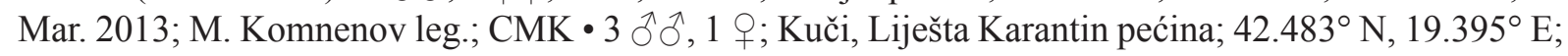
575 m a.s.l.; 23 Mar. 2013; M. Komnenov leg.; CMK • 2 + ; ; Kučke Mt., Kučka korita, Radeča, beech forest; $42.500^{\circ} \mathrm{N}, 19.534^{\circ} \mathrm{E} ; 22 \mathrm{Jul}$. 2010; M. Komnenov leg.; CMK • 1 juv. assigned tentatively; Kučka korita, Jama J008; $42.4747^{\circ}$ N, $19.5247^{\circ}$ E; 1350 m a.s.1.; 10 Nov. 2015; A. Kirin leg.; CBSS (Ar 4178).

ALBANIA - Shkodra • 1 đ̊; K2 Cave nr. Kozhnje [Kozhnja]; $42.457^{\circ}$ N, $19.607^{\circ}$ E; 30 Aug. 1996; "P.B." leg.; NMNHS. - Kukës • 1 đ̊; Hekurave Mt., Qereç-Mulaj, Zeze Cave; $42.362^{\circ}$ N, $19.930^{\circ}$ E; 18

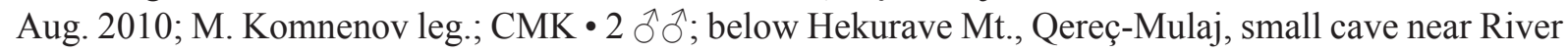
Kuqit; $42.364^{\circ}$ N, $19.933^{\circ}$ E; 17 Aug. 2010; M. Komnenov leg.; CMK • 1 q; Prokletije, Curaj i Epërm, Nëngurit Cave; $42.339^{\circ}$ N, $19.927^{\circ}$ E; 19 Aug. 2010; M. Komnenov leg.; CMK.

\section{Redescription}

Male (type locality; ZFMK (Ar 22201))

MEAsurements. Total body length 5.5, carapace width 2.15. Distance PME-PME $150 \mu \mathrm{m}$; diameter PME $130 \times 160 \mu \mathrm{m}$; distance PME-ALE $30 \mu \mathrm{m}$; diameter AME $60 \mu \mathrm{m}$; distance AME-AME $20 \mu \mathrm{m}$. Leg 1: $50.6(13.7+0.8+13.3+19.6+3.2)$, tibia $2: 9.7$, tibia $3: 8.0$, tibia $4: 8.5$; tibia $1 \mathrm{~L} / \mathrm{d}: 65$.

COLOR (in ethanol). Carapace pale whitish to ochre-yellow; ocular area, clypeus, and posteriorly between furrows slightly darker (light brown); sternum light brown, with dark brown margins and irregular dark triangular radial marks; legs ochre yellow to light brown, without dark rings, without dark lines; abdomen mostly monochromous pale gray, only above spinnerets and ventrally in gonopore area some dark marks. 
Body. Habitus as in Fig. 3. Ocular area slightly raised. Carapace with deep median pit and pair of shallow furrows diverging from pit to posterior margin of carapace (Fig. 91). Clypeus unmodified. Sternum wider than long (1.40/1.05), unmodified. Abdomen oval, pointed at spinnerets.

Chelicerae. Very similar to S. skotophilus (cf. Figs 51-52; see also Fig. 93), with pair of frontal apophyses set with 3 and 4 modified hairs, respectively, with 16 and 18 modified hairs each on frontal face (Fig. 94); with stridulatory ridges (Fig. 95).

PALPS. In general as in S. skotophilus (cf. Figs 6-8); coxa with retrolateral hump; trochanter barely modified; femur with distinct retrolateral process proximally, with stridulatory pick (modified hair) proximally on prolateral side (Fig. 103); femur-patella joints shifted towards prolateral; tibia very large, with two trichobothria; tibia-tarsus joints shifted towards retrolateral; tarsal organ exposed (Fig. 104); procursus dorsally with $\sim 8$ curved hairs (distal third curved 'upwards'), with strong hump on prolateral side. Procursus tip (Figs 77, 99-102) with slightly projecting dorsal sclerite, strong hinged ventral sclerite (flat, wider in ventral than in lateral view), and membranous central part with brush of hair-like processes on retrolateral side, with narrow and weakly sclerotized process on prolateral side bordering ventral sclerite. Genital bulb (Figs 79-82) with basal sclerite connecting to tarsus, small transparent dorsal process (Fig. 98), and main sclerite consisting of retrolateral process and dorsal process; retrolateral process with bifid tip, distal part on ventral side thickened and heavily sclerotized, possibly with glandular pores distally (Figs 105-106); dorsal process with two finger-shaped tips, ventral tip simple, retrolateral tip with additional strong protrusion on ventral side.

Legs. With short spines ventrally on femur 1 in two rows in distal third, prolateral row $\sim 19$ spines, retrolateral row $\sim 24$ spines; without curved hairs; few vertical hairs; retrolateral trichobothrium of tibia 1 at $4 \%$; prolateral trichobothrium absent on tibia 1 , present on other tibiae; tarsus 1 with irregular platelets rather than with distinct pseudosegments.

Male (variation)

Some specimens with more and larger dark marks dorsally on abdomen (posterior third), few specimens without abdominal marks; dark pattern on sternum slightly variable. Tibia $1(\mathrm{~N}=29)$ : 11.3-14.5 (mean 12.9); tibia $2(\mathrm{~N}=35)$ : 8.1-10.8 (mean 9.6); spines on femur $1(\mathrm{~N}=23)$ : $24-53$ (mean 40); modified hairs on cheliceral basis $(\mathrm{N}=70)$ : 11-22 (mean 14.7); modified hairs on cheliceral apophysis $(\mathrm{N}=69)$ : 2-4 (mean 3.0); chelicerae maximum width $(\mathrm{N}=35)$ : 0.72-0.84 (mean 0.79); procursus length $(\mathrm{N}=35)$ : 1.22-1.46 (mean 1.37); bulbal measures A-E: $\mathrm{A}(\mathrm{N}=17)$ : 0.34-0.43 (mean 0.37); $\mathrm{B}(\mathrm{N}=17): 0.10-0.15$ (mean 0.13); $\mathrm{C}(\mathrm{N}=17): 0.24-0.27$ (mean 0.25); $\mathrm{D}(\mathrm{N}=17)$ : 0.25-0.29 (mean 0.27); $\mathrm{E}(\mathrm{N}=8): 0.82$ 0.90 (mean 0.88); epiandrous spigots $(\mathrm{N}=25)$ : 5-9 (mean 6.2).

Males from SE Montenegro (Podgorica) and NW Albania differ slightly from other specimens, most conspicuously in bulbal measure C (smaller: 0.19-0.22).

\section{Female}

In general similar to male (Fig. 3), but femur 1 without spines, with pair of indistinct processes posteriorly on carapace (Fig. 107) and pair of opposing poorly visible plates on abdomen, distance between processes/plates: 1.1. Tibia $1(\mathrm{~N}=28)$ : $8.3-15.3$ (mean 12.9); tibia $2(\mathrm{~N}=30): 6.0-10.9$ (mean 9.3); epigynum width $(\mathrm{N}=31)$ : $0.94-1.50$ (mean 1.27); epigynum length $(\mathrm{N}=31)$ : $0.50-0.70$ (mean 0.62 ); epigynal measure $\mathrm{F}(\mathrm{N}=31)$ : $0.22-0.40$ (mean 0.30 ). Epigynum anterior plate semicircular, weakly protruding, with weak transversal furrows (Figs 85-88, 111), with internal structures visible in uncleared specimens: round anterior structure, short sclerites originating from posterior margin and slightly converging anteriorly; posterior plate short but wide. Internal genitalia (Figs 83-84, 149-150) with distinctive ventral arc (Fig. 155; approximately as long as wide), with large pore plates (Fig. 84). 
Females from SE Montenegro (Podgorica) and NW Albania tend to have a slightly larger epigynal measure F: 0.37-0.43 $(\mathrm{N}=6)$ (cf. Figs 89-90).

\section{Distribution}

Known from Montenegro and NW Albania (Fig. 168).

Stygopholcus photophilus Senglet, 1971

Figs 4-5, 9-11, 113-144, 151-152, 156

Stygopholcus photophilus Senglet, 1971: 356, figs 44-54.

Hoplopholcus kratochvili - Brignoli, 1971: 259, figs 1-6. — Deltshev 1985: 43. Synonymized by Brignoli (1976).

Hoplopholcus photophilus - Brignoli 1976: 561.

Stygopholcus photophilus - Senglet 2001: 47, 65, figs 15-20, 23-25, 28.

\section{Diagnosis}

Easily distinguished from known congeners by many characters, including: distinct dark marks on entire abdomen (Figs 4-5), also ventrally; dark rings on femora and tibiae, dark lines on femora (Figs 127-128); male chelicerae with strongly sculptured median margins (Figs 130-131); larger number of modified hairs on male cheliceral base (Fig. 162; with little overlap); male palpal femur distally more strongly widened (Fig. 11); palpal tibia widest in distal half (Fig. 11); procursus distally with strongly projecting dorsal sclerite (Figs 113-114); dorsal bulbal process not divided into two separate tips (Figs 115, 118); spines on male femur 1 in single row; male tibia 1 much longer than tibia 2 (tibia 1/tibia $2>1.40$; in other species < 1.40; Fig. 165); strong transversal ridges on anterior part of epigynum (Fig. 144); relatively longer epigynum (epigynum width/length: usually $<1.7$, in other species usually $>1.7$, with little overlap; Fig. 163); female without stridulatory ridges on chelicerae (Fig. 134), and without stridulatory apparatus between prosoma and abdomen.

Type material of Stygopholcus photophilus

\section{Holotype}

GREECE - Thessaly $•{ }^{\lambda}$, examined; Larissa, Omolion (Tembe) [Omolio, Tempi Valley]; $39.900^{\circ} \mathrm{N}$, $22.628^{\circ}$ E; 17 Jun. 1970; A. Senglet leg.; MHNG.

\section{Paratypes}

GREECE - Thessaly • 27 ठิ $\widehat{\partial}, 14$ 우 ( 3 vials), examined; same collection data as for holotype; MHNG.

For further paratypes from other localities, see below.

Type material of Hoplopholcus kratochvili

Holotype

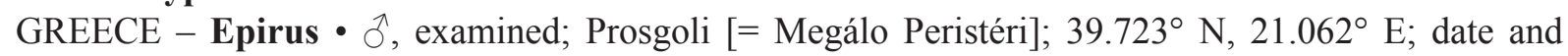
collector unknown; ZMB (9077).

\section{Paratypes}

GREECE - Epirus • 1 ô, 1 juv. (2 vials), examined; same collection data as for holotype; ZMB (9077)

$\bullet 1$, examined; same collection data as for holotype; MCVR. 


\section{Other material examined}

GREECE - Thessaly $\bullet 2 \hat{\partial} \widehat{\partial}, 3$ + $\propto$; Monte Ossa above Ampelakia, unnamed cave near church Profitis

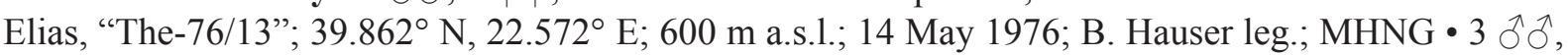
2 우; same locality as for preceding, "Kri 78/27”; 10 Apr. 1978; B. Hauser leg.; MHNG・1 ð; Monte
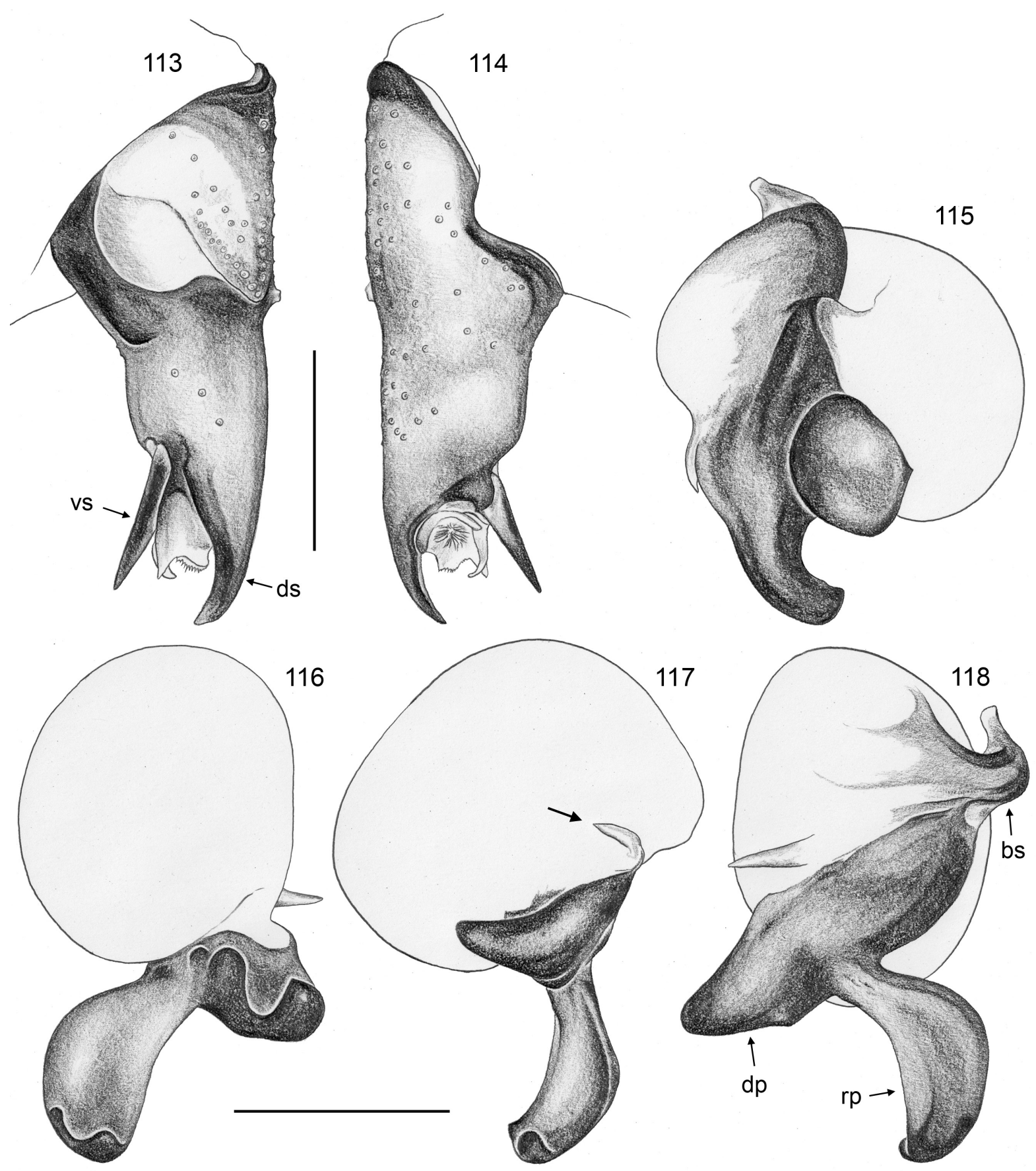

Figs 113-118. Stygopholcus photophilus Senglet, 1971; male from Episkopi, Crete (ZFMK (Ar 22205)). 113-114. Left tarsus and procursus, prolateral and retrolateral views. 115-118. Left genital bulb, retrolateral, ventral, prolateral-distal, and dorso-distal views; arrow points at weakly sclerotized process. Abbreviations: $\mathrm{bs}=$ basal sclerite; $\mathrm{dp}=$ dorsal process; $\mathrm{ds}=$ dorsal sclerite; $\mathrm{rp}=$ retrolateral process; vs $=$ ventral sclerite. Scale bars: $0.5 \mathrm{~mm}$. 
Ossa, near Spilia, Tsari Tripa cave on Mt. Psylodendron [= Spilaio Nymfon], "Kri-78/30"; 39.821 N, $22.641^{\circ}$ E; 11 Apr. 1978; B. Hauser leg.; MHNG • 2 우우 ; ravine near Stomio (“Ossa-Geb., Stomio, Bachgraben”), "TH 234"; $39.86^{\circ}$ N, $22.72^{\circ}$ E; 22 Sep. 1995; K. Thaler and B. Knoflach leg.; ZFMK

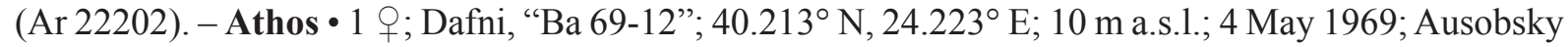
leg.; ZFMK (Ar 22203) • 1 §, 1 क , 1 juv.; Mount Athos, Agia Anna monastery, Lost Glasses Cave; $40.14^{\circ}$ N, $24.30^{\circ}$ E; 8 Sep. 2012; A. Zhalov leg.; NMNHS. - Central Macedonia • 1 ơ; Chalkididi, Sithonia, $1 \mathrm{~km} \mathrm{~W}$ of Zografou, humid stream valley with Osmunda, under logs and stones; $40.1702^{\circ} \mathrm{N}$, 23.8545 E; 20 m a.s.1.; 22 Aug. 2009; S. Huber and A. Schönhofer leg.; SMF. - Eastern Macedonia

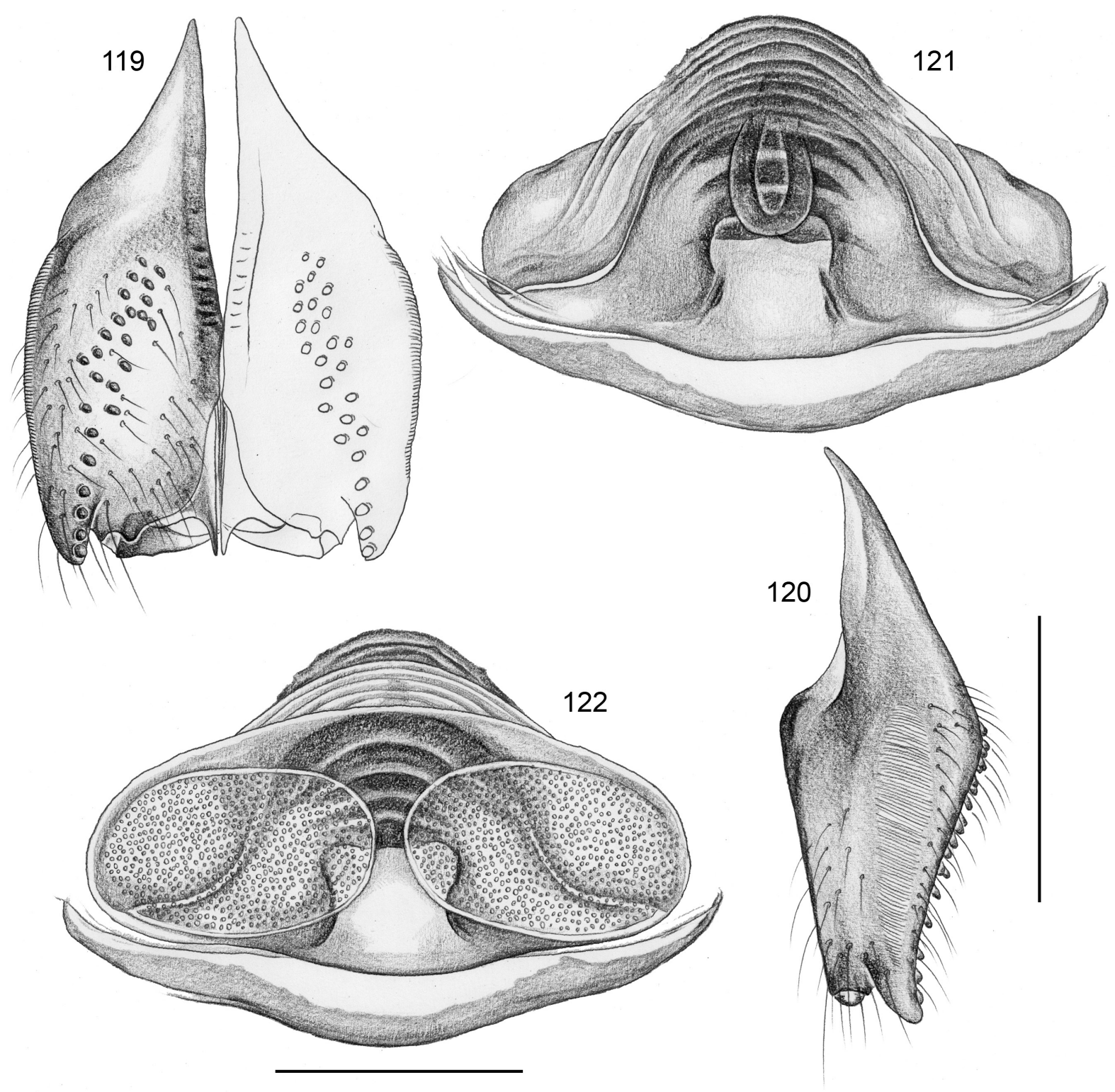

Figs 119-120. Stygopholcus photophilus Senglet, 1971; male from Episkopi, Crete (ZFMK (Ar 22205)), female from Choumerion/Pérama, Crete (MHNG). 119-120. Male chelicerae, frontal and lateral views. 121-122. Cleared female genitalia, ventral and dorsal views. Scale bars: $0.5 \mathrm{~mm}$. 
and Thrace $\bullet 2 \overbrace{}^{\lambda} \partial^{\lambda}, 5$ 우; Thasos, Panagia, Drakotrypa cave; $40.732^{\circ} \mathrm{N}, 24.732^{\circ} \mathrm{E} ; 28$ Dec. 1982; P. Beron and S. Andreev leg.; NMNHS 11 خ; Kavala, Zygos, Aghia Helleni cave [Spilaio Agias Elenis];

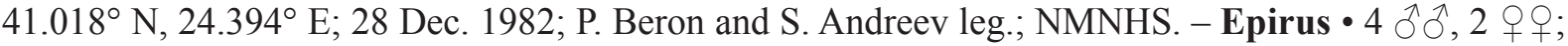

123
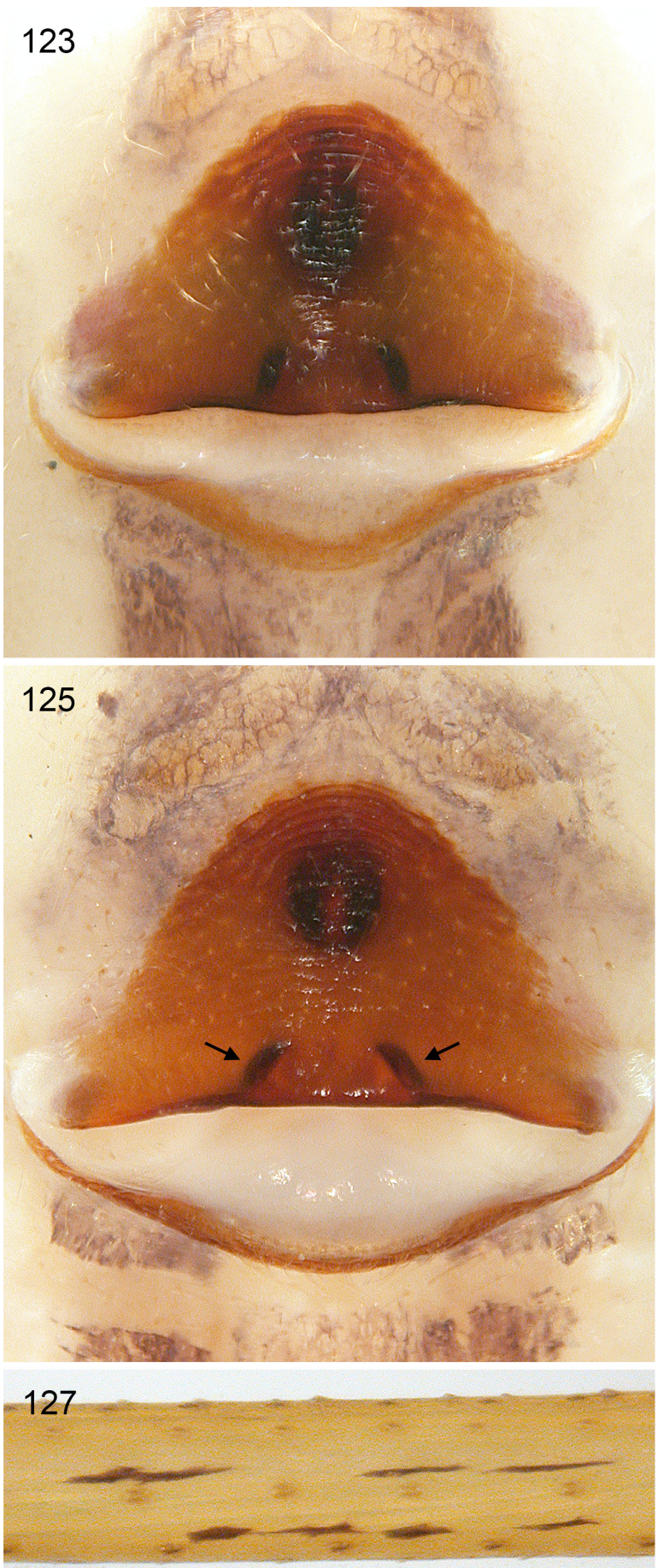

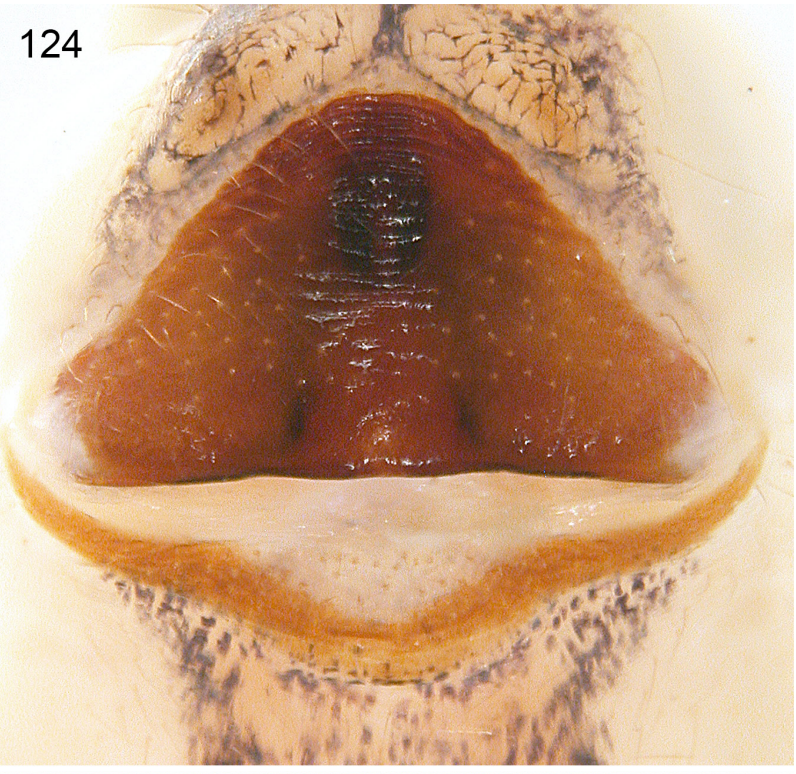

126

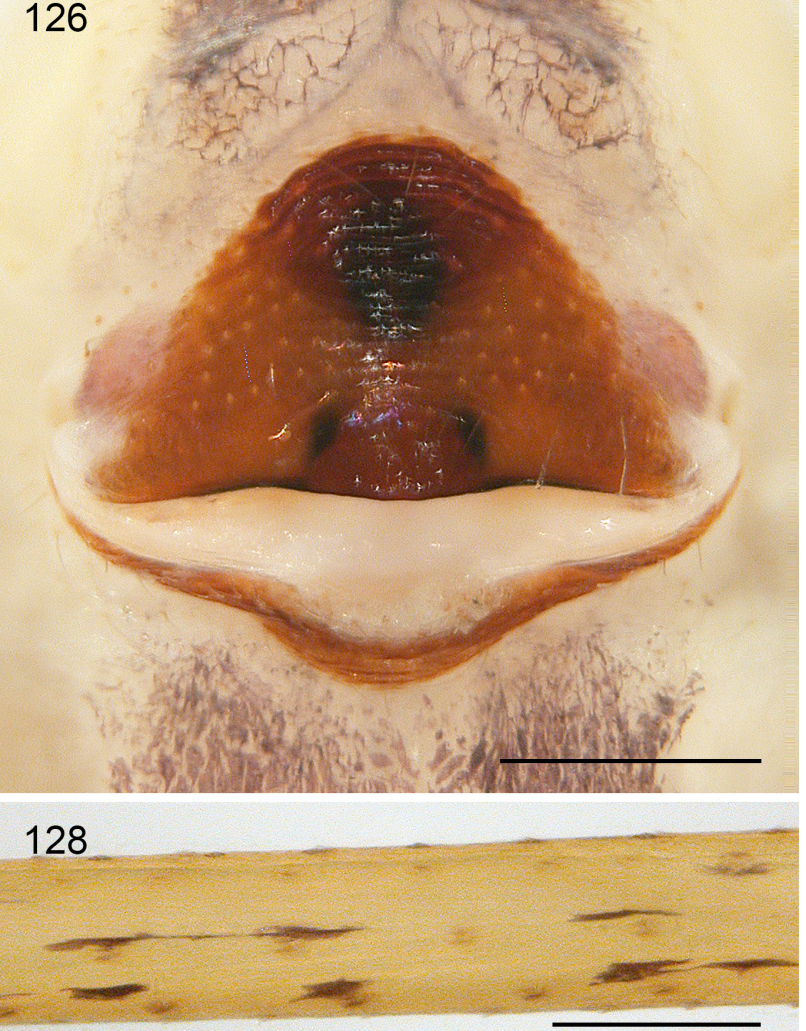

Figs 123-128. Stygopholcus photophilus Senglet, 1971; epigyna, ventral views, and details of male femora 2 and 3, dorsal views. 123. Thessaly, Omolio, Tempi Valley (MHNG). 124. Corfu, Loutses (MHNG). 125. Peloponnese, Agios Dimitrios/Lefktro (MHNG); arrows point at internal sclerites. 126128. Crete, Episkopi (ZFMK (Ar 22205)). Scale bars: $123-126=0.5 \mathrm{~mm} ; 127-128=0.2 \mathrm{~mm}$. 

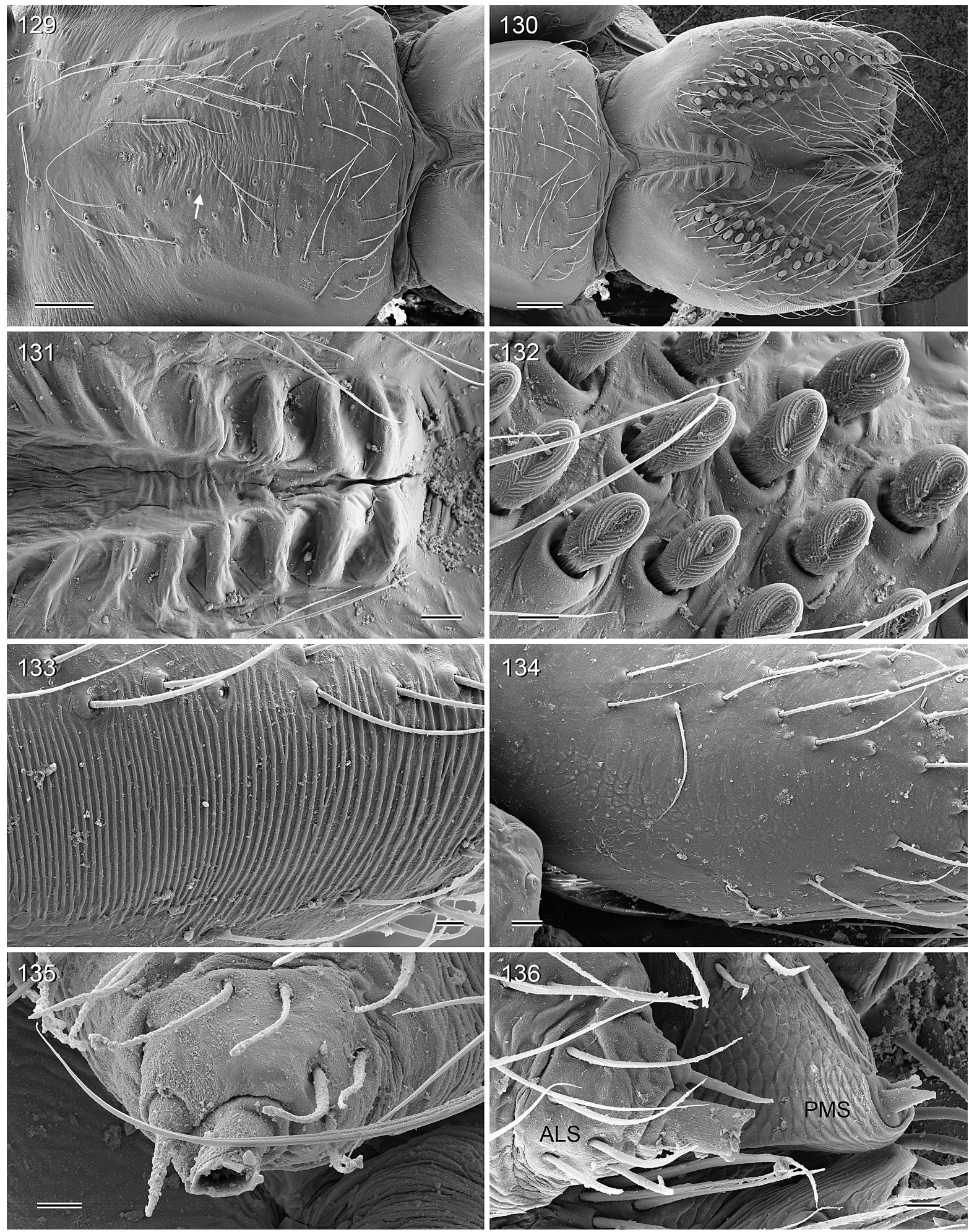

Figs 129-136. Stygopholcus photophilus Senglet, 1971; male and female from Choumerion/Pérama, Crete (MHNG). 129. Male clypeus, frontal view; arrow points at modified median area. 130. Male chelicerae, frontal view. 131. Modified area medially on male chelicerae. 132. Modified hairs frontally on male chelicerae. 133. Stridulatory files on male chelicerae. 134. Absence of stridulatory files on female chelicerae. 135. Male ALS. 136. Female anterior lateral spinneret (ALS) and posterior median spinneret (PMS). Scale bars: $129-130=100 \mu \mathrm{m} ; 131,134-136=20 \mu \mathrm{m} ; 132-133=10 \mu \mathrm{m}$. 

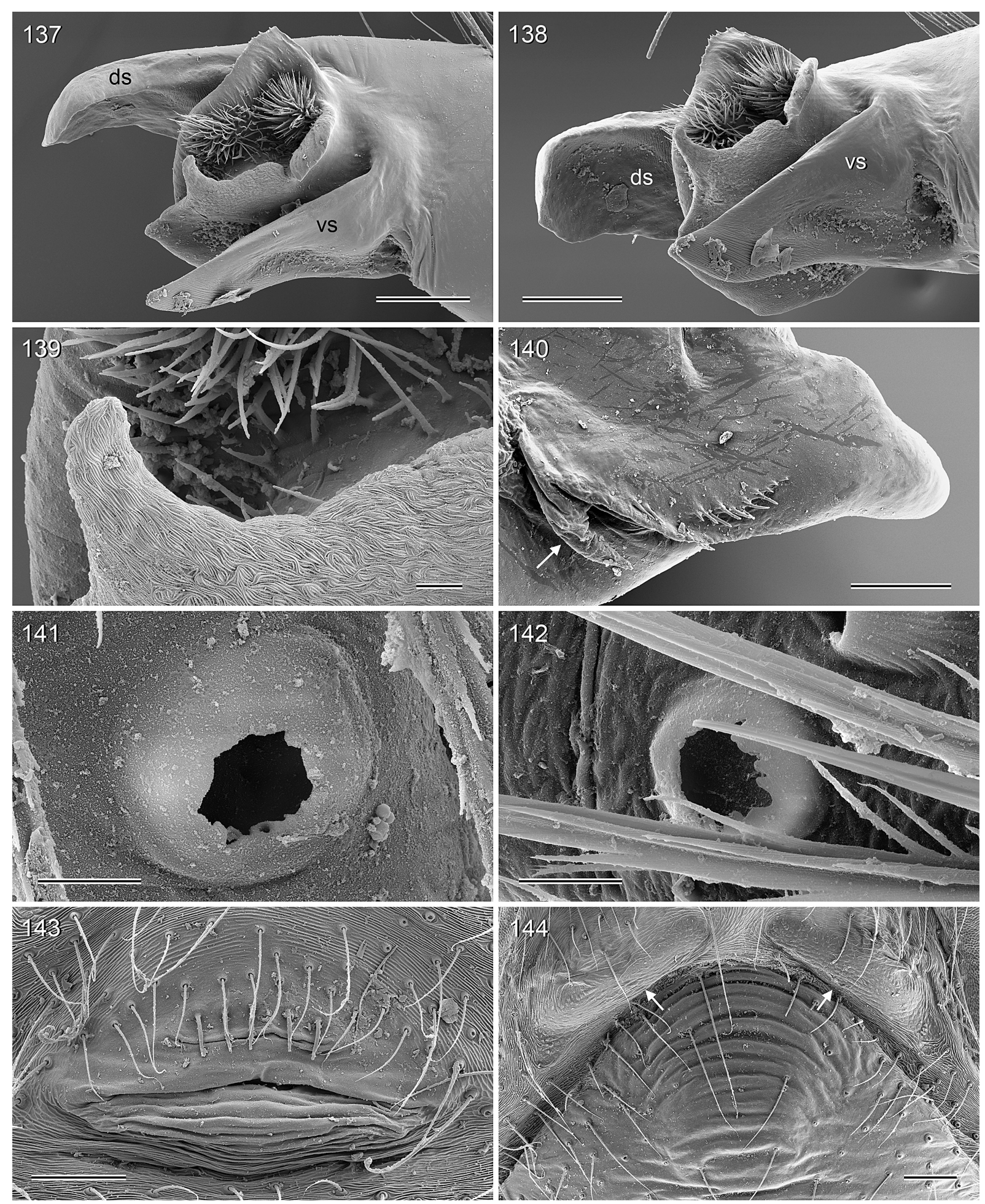

Figs 137-144. Stygopholcus photophilus Senglet, 1971; male and female from Choumerion/Pérama, Crete (MHNG). 137-138. Tip of left procursus, retrolateral (slightly ventral) and ventral views. 139. Detail of previous figure. 140. Dorsal bulbal process and transparent bulbal process (arrow); note hair-like structures on dorsal bulbal process. 141. Female palpal tarsal organ. 142. Tarsal organ on female tarsus 4. 143. Male gonopore and epiandrous spigots. 144. Anterior part of epigynum showing strong ridges; arrows point at pair of anterior bulges. Abbreviations: ds $=$ dorsal sclerite; vs $=$ ventral sclerite. Scale bars: $137-138,140,143-144=100 \mu \mathrm{m} ; 139,141-142=10 \mu \mathrm{m}$. 

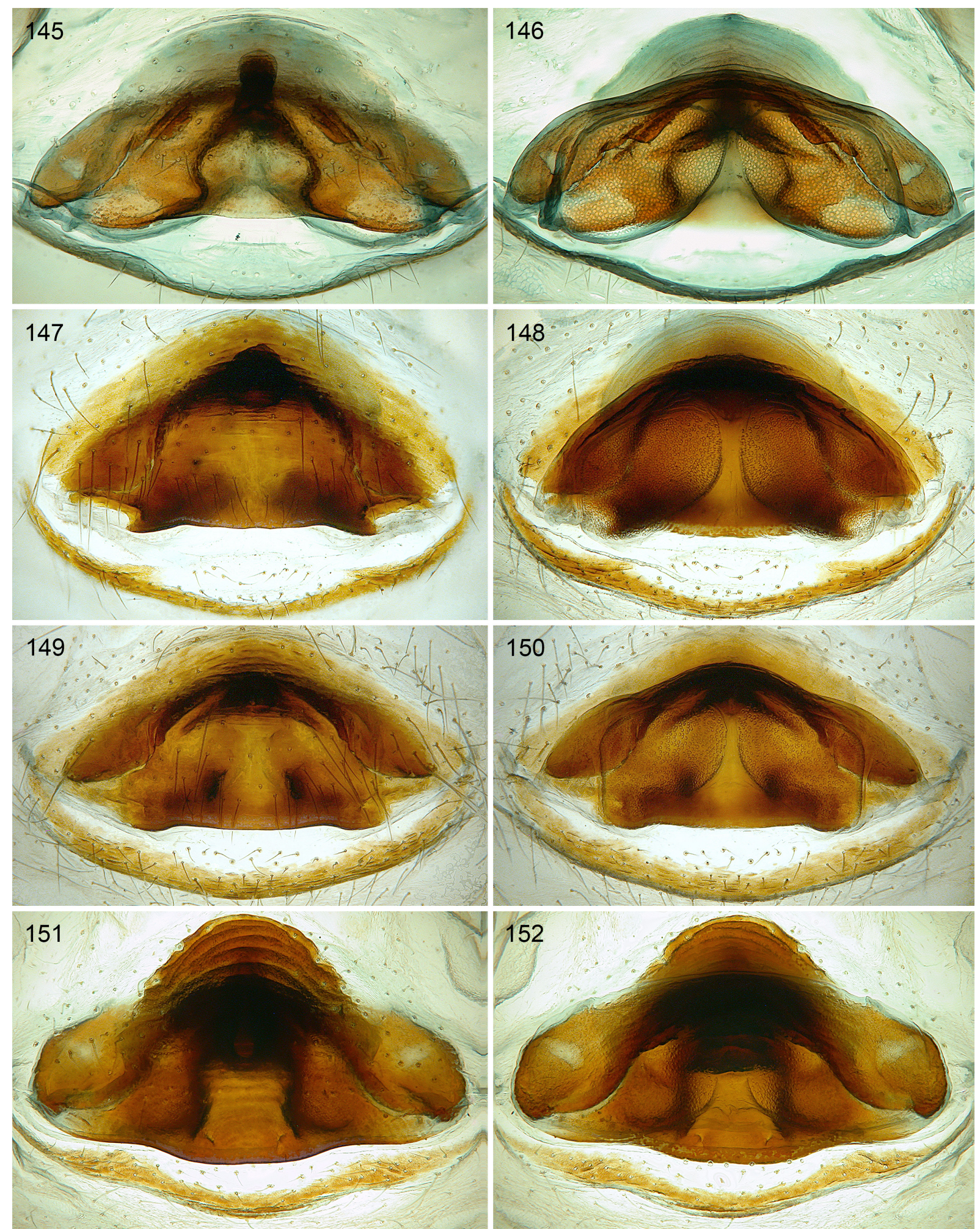

Figs 145-152. Stygopholcus Kratochvíl, 1932; cleared female genitalia, ventral and dorsal views. 145-146. S. absoloni (Kulczyński, 1914); from Zavala, Bosnia and Herzegovina (MHNG). 147148. S. skotophilus Kratochvíl, 1940; from Pećina u Ivici, Montenegro ((ZFMK Ar 22196)). 149150. S. montenegrinus Kratochvíl, 1940; from Golubinja pećina, Montenegro (CMK). 151-152. S. photophilus Senglet, 1971; from Choumerion/Pérama, Crete (MHNG). 
Monodendri, dry valley before monastery, oak and hard-leafed forest, under stones and wood; $39.883^{\circ} \mathrm{N}$, $20.749^{\circ}$ E; 1000 m a.s.1.; 14 Aug. 2009; S. Huber and A. Schönhofer leg.; SMF. - Peloponnese • 1 万’; Ag. Taxiarchi, $12 \mathrm{~km} \mathrm{~W}$ of Leonidion, cellar of church ruin; $37.150^{\circ} \mathrm{N}, 22.760^{\circ} \mathrm{E} ; 29 \mathrm{Sep} .2006 ; \mathrm{H}$.

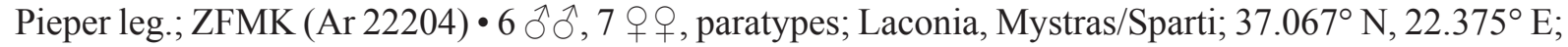

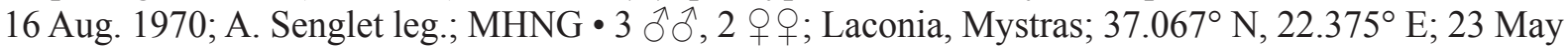

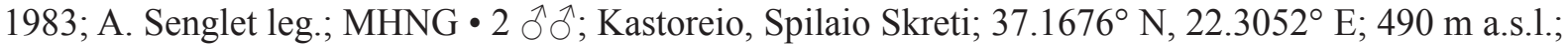
5 Dec. 2016; C. Cenni leg.; MCVR • 5 $\widehat{\jmath}, 5$ 우, 6 juvs, paratypes; Messinia, Agios Dimitrios/Lefktro

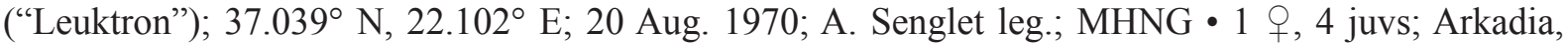

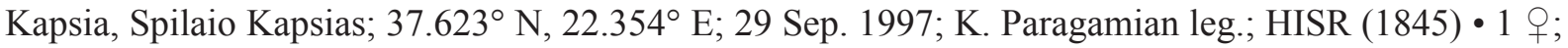
Arkadia, Leonidio, Spilaio Dionysou; $37.1485^{\circ}$ N, $22.8185^{\circ}$ E; 19 Sep. 1997; K. Paragamian leg.; HISR

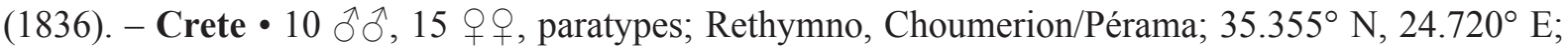

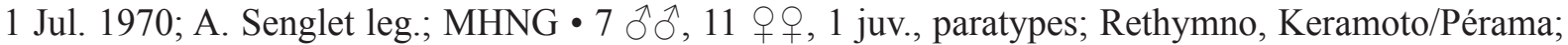
$35.333^{\circ}$ N, $24.740^{\circ}$ E; 2 Jul. 1970; A. Senglet leg.; MHNG • 1 ô, 1 q; Rethymno, Archaia Eleftherna;

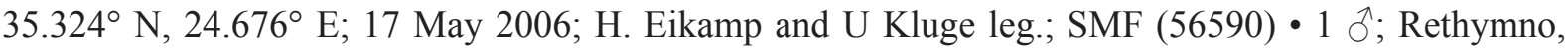
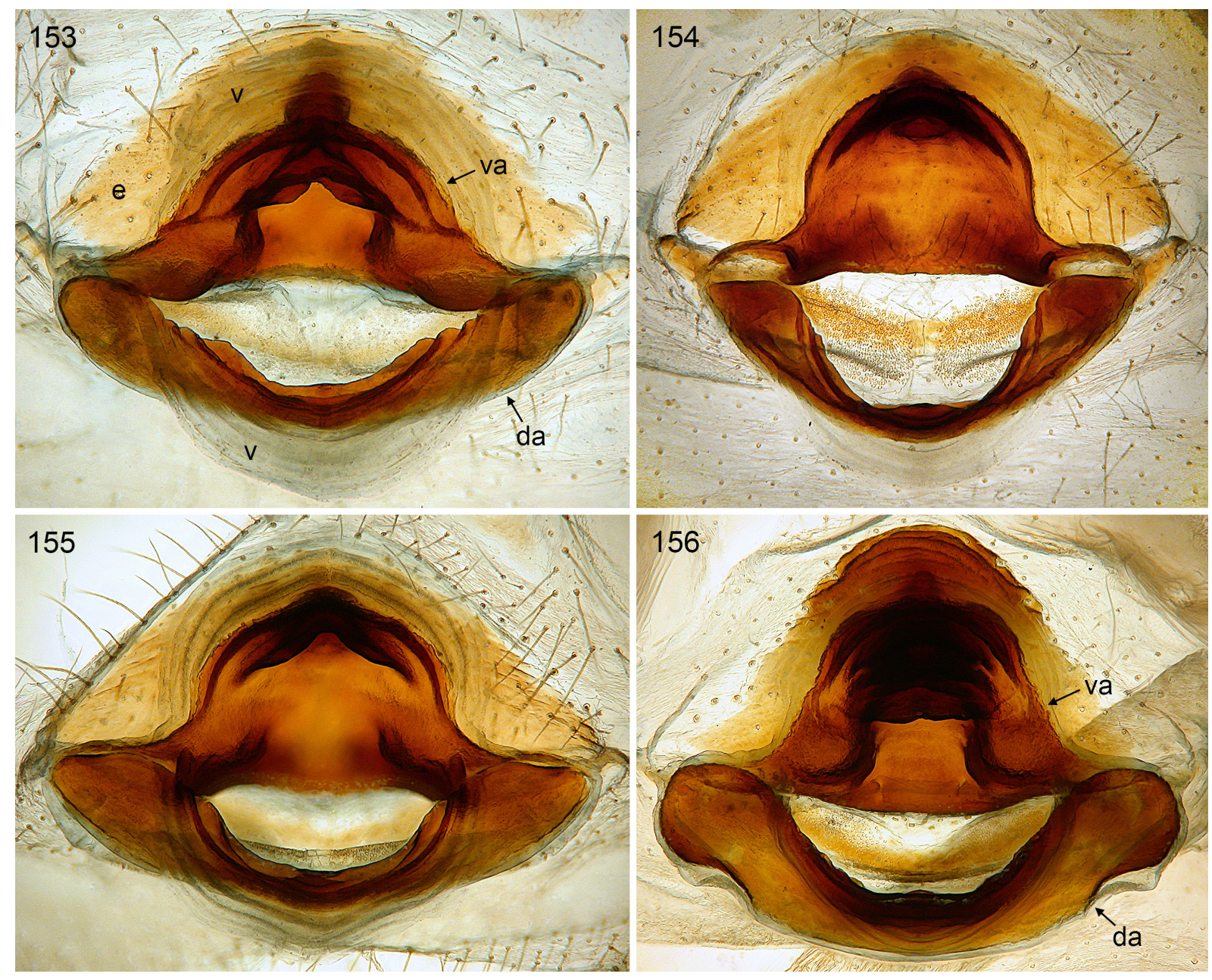

Figs 153-156. Stygopholcus Kratochvíl, 1932; cleared female genitalia in dorsal views; dorsal sclerotized arcs tilted towards the back. 153. S. absoloni (Kulczyński, 1914); from Balićeva špilja, Croatia (ZFMK (Ar 22195)). 154. S. skotophilus Kratochvíl, 1940; from Pećina u Ivici, Montenegro (ZFMK (Ar 22196)). 155. S. montenegrinus Kratochvíl, 1940; from Studenačka pećina, Montenegro (ZFMK (Ar 22201)). 156. S. photophilus Senglet, 1971; from Choumerion/Pérama, Crete (MHNG). Abbreviations: $\mathrm{da}=$ dorsal arc; $\mathrm{e}=$ epigynal plate; $\mathrm{v}=$ 'valve'; $\mathrm{va}=$ ventral arc. 
Myloi, Spilaio Skoteinospilios; 35.336 N, 24.505 E; 1 Mar. 1987; K. Paragamian leg.; HISR (1640)

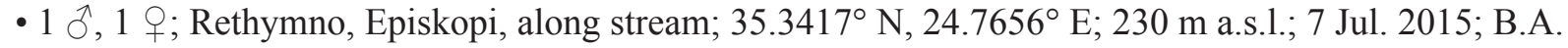

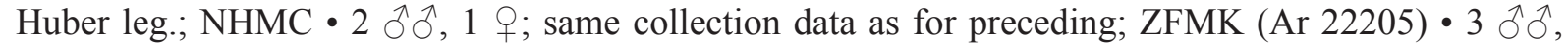
2 우오, in pure ethanol; same collection data as for preceding; ZFMK (Cre19) 2 ô, 2 juvs; Irakleion,

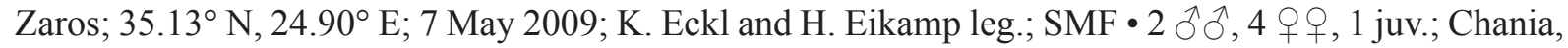
Topolia, Spilaio Agias Sofias; $35.417^{\circ}$ N, 23.683 E; 30 Dec. 2001; K. Paragamian leg.; HISR (2008)

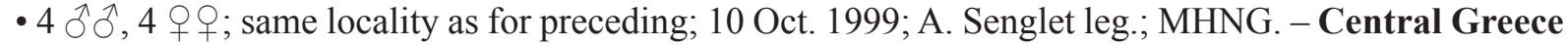

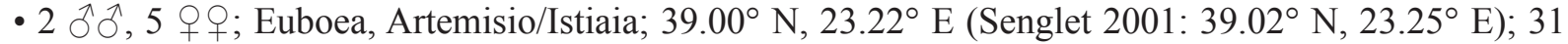

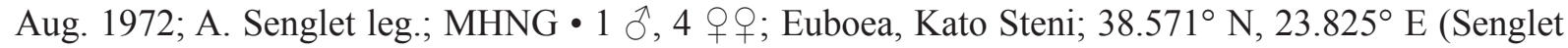

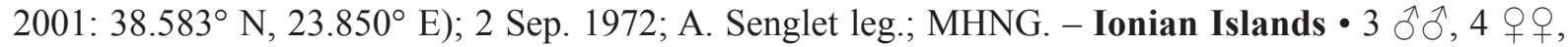
2 juvs; Lefkada, waterfalls $3 \mathrm{~km} \mathrm{~W}$ of Nidri, rocky caves and mossy stones; $38.7263^{\circ} \mathrm{N}, 20.6858^{\circ} \mathrm{E}$; 200 m a.s.l.; 27 Mar. 2006; A. Schönhofer leg.; SMF • 1 đ, 1 q; Kefallonia, Lake Avithos, lakeside

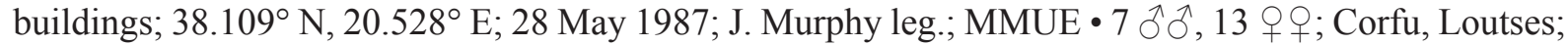
$39.786^{\circ}$ N, $19.880^{\circ}$ E; 20 Sep. 1972; A. Senglet leg.; MHNG 1 ○’; Corfu, S of Loutses, Spilaio Megali Grava / Peristerograva, on ground of cave, under stones; $39.777^{\circ} \mathrm{N}, 19.890^{\circ} \mathrm{E} ; 350 \mathrm{~m}$ a.s.1.; 22 Mar. 2006; A. Schönhofer leg.; SMF 6 ○ð, 2 우; same locality as for preceding, ("Halbhöhle am Nordabhang

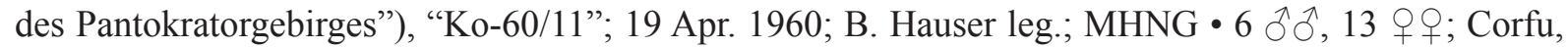
Pantocrator, Spilaio Katsampa ("Katsaba cave") near Strinilas, "Pel74/19"; 39.744 N, $19.839^{\circ}$ E; 12 May 1974; B. Hauser leg.; MHNG.
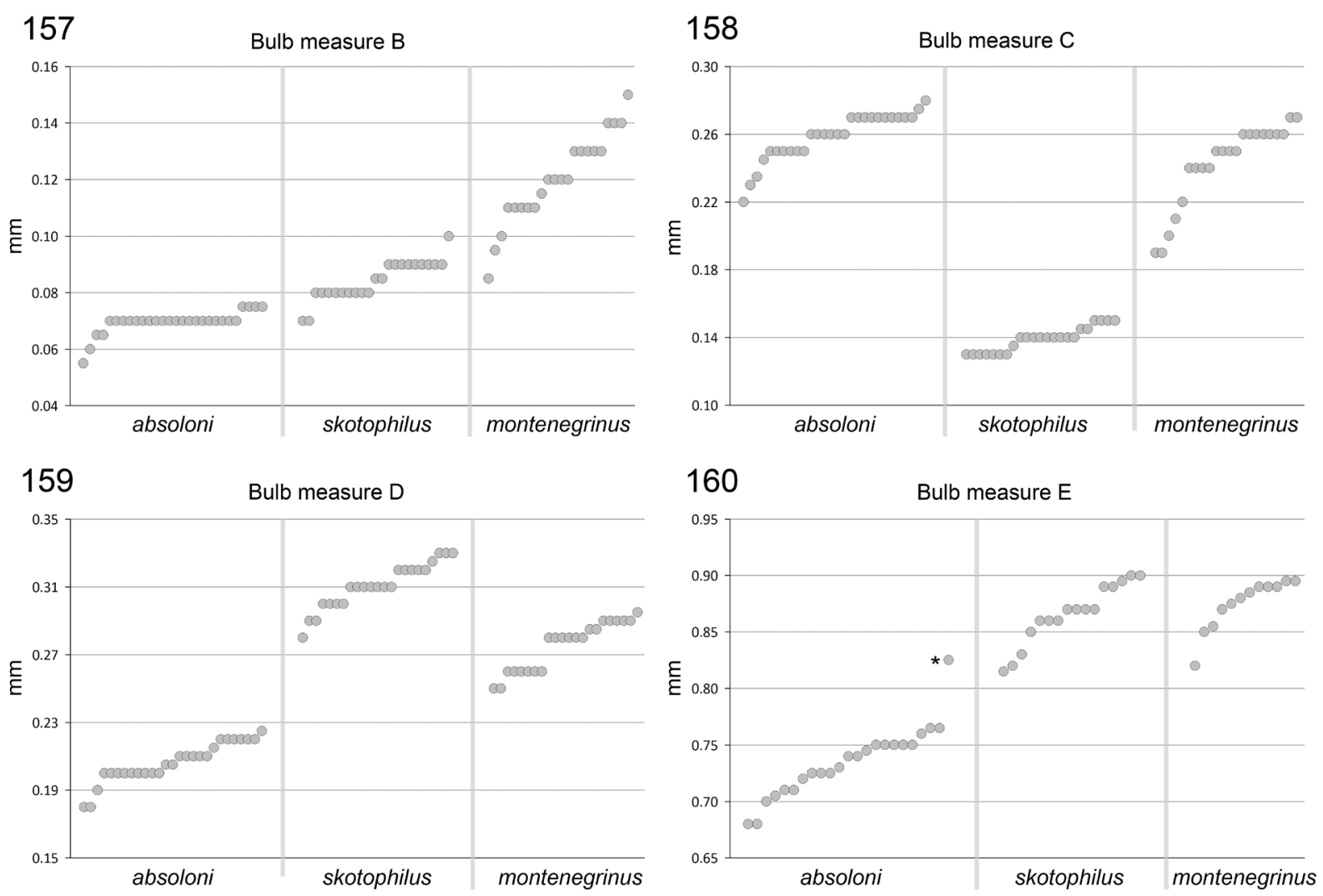

Figs 157-160. Bulbal measures B-E for the three species of the 'northern clade' of Stygopholcus Kratochvíl, 1932 (cf. Figs 15-18). Bulbal measure A is strongly correlated with measure C and therefore not shown. Bulbal measures sorted from smallest to biggest for each species of the 'northern clade'. The bulb of $S$. photophilus was not measured (it differs strongly in shape, and measure D is inapplicable). Asterisk in Fig. $160=$ dubious specimen from Jama na Brezdanu (see text). 
ALBANIA - Gjirokastër • 1 §, 1 o; Përmet, near Petran, artificial gallery; $40.208^{\circ}$ N, $20.419^{\circ}$ E; 12 May 1995; B. Petrov and R. Stoev leg.; NMNHS.

Credible published records (not examined)

GREECE - Epirus - 1 O; $11 \mathrm{~km} \mathrm{SE}$ of Konitsa, in gorge of river Voithomatis ["Bogsomatis", side river of Aoos river], "Ep-73/48”; 40.019 N, 20.785 E; 30 Apr. 1973; V. Mahnert leg.; (Brignoli 1976); MHNG(?).

ALBANIA - (the following material could not be found, C. Deltshev pers. comm., 30 Sep. 2020) Durrës • ${ }^{\top}$ 우 (not specified); Durrës; 41.3 N, 19.5 E; 26 May 1993; P. Stoev and D. Zapryanova leg.
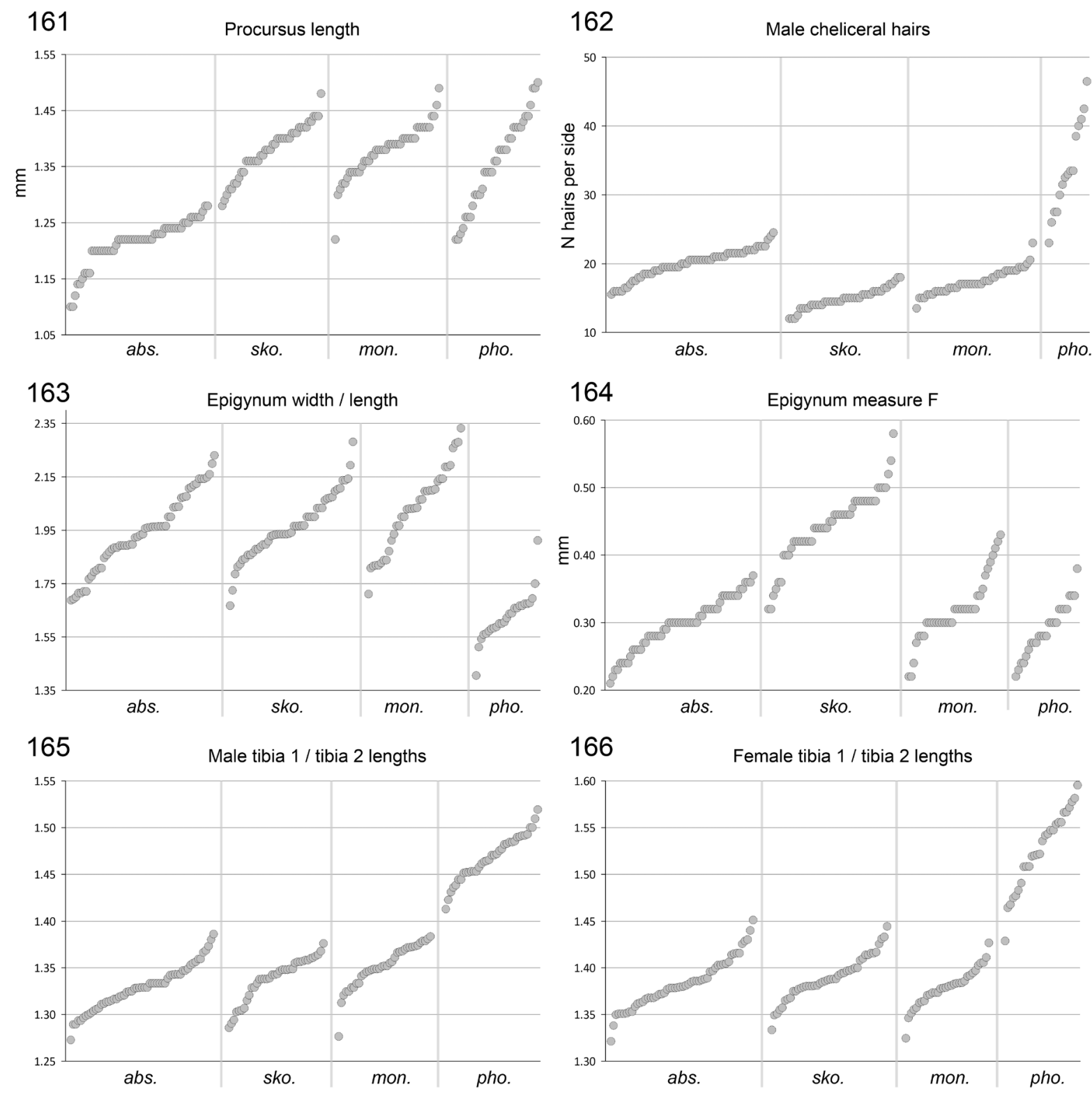

Figs 161-166. Procursus length, number of modified hairs on each male chelicera, epigynum width/ length, epigynum measure F (cf. Fig. 21), and relationships between tibia 1 and tibia 2 lengths in males and females. Measures/counts sorted from smallest to biggest for each species. Abbreviations: abs. $=$ S. absoloni; sko. $=$ S. skotophilus; mon. $=$ S. montenegrinus; pho. $=$ S. photophilus . 
(Deltshev et al. 2011). - Korcë • $\widehat{O}$ 우 (not specified); Cave 2 near Leskovik; $40.150^{\circ} \mathrm{N}, 20.600^{\circ} \mathrm{E}$; 1 Jun. 1994; T. Ivanova leg. (Deltshev et al. 2011). - Gjirokastër • $\widehat{\jmath}$ 우 (not specified), Dragot, Vijoshe

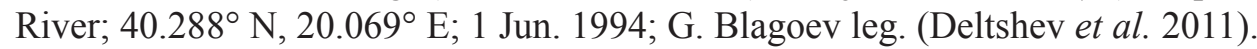

\section{Unidentified and unspecified localities}

GREECE • 1 \%; Crete, "Chassia" [misspelling on copied label?], "RII/8"; May 1926; Roewer leg.; SMF

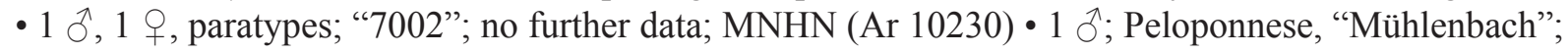
6 Oct. 1980; Malicky leg.; SMF.

\section{Redescription}

Male (Crete; ZFMK (Ar 22205))

MEASUREMEnTs. Total body length 4.3, carapace width 1.5. Distance PME-PME $130 \mu \mathrm{m}$; diameter PME $120 \times 140 \mu \mathrm{m}$; distance PME-ALE $30 \mu \mathrm{m}$; diameter AME $60 \mu \mathrm{m}$; distance AME-AME $30 \mu \mathrm{m}$. Leg 1: $40.4(10.7+0.7+10.5+16.0+2.5)$, tibia $2: 6.9$, tibia $3: 5.1$, tibia $4: 5.7$; tibia $1 \mathrm{~L} / \mathrm{d}: 75$.

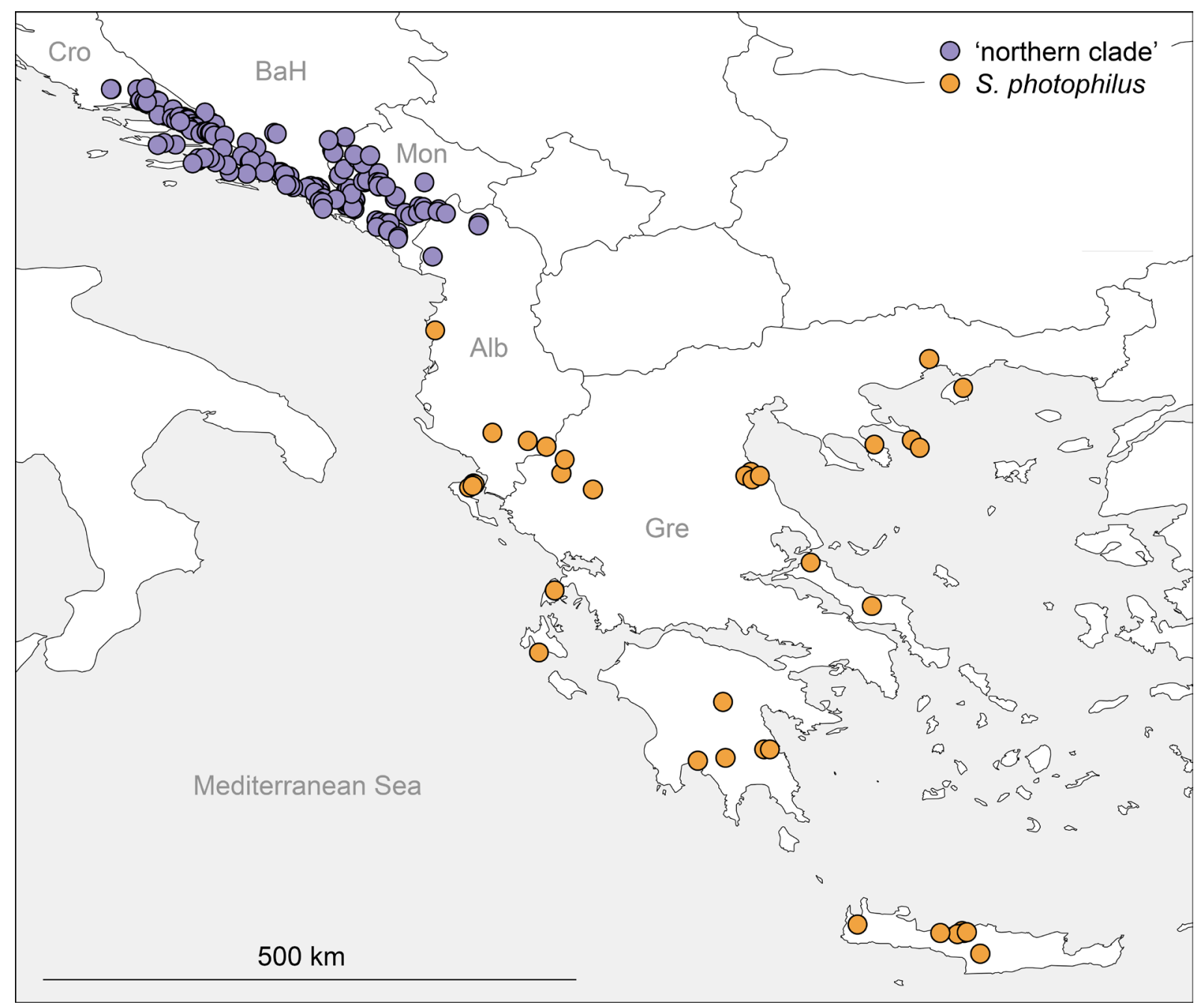

Fig. 167. Known distribution of Stygopholcus Kratochvíl, 1932. For the 'northern clade', see Fig. 168. Abbreviations: $\mathrm{Alb}=$ Albania; $\mathrm{BaH}=$ Bosnia and Herzegovina; Cro $=$ Croatia; Gre $=$ Greece; Mon $=$ Montenegro. 
Color (in ethanol). Carapace pale whitish to ochre-yellow, ocular area and clypeus slightly darker (light brown), ocular area posteriorly with darker brown V-mark; sternum very dark brown, almost black; legs ochre yellow, with darker (light brown) rings on femora (subdistally) and tibiae (proximally and subdistally), with short dark longitudinal lines dorsally on femora (Figs 127-128); abdomen ochregray, with distinct dorsal pattern consisting of brown band around heart and dorsal and lateral marks in posterior half; with white internal marks; ventrally with wide dark brown band, interrupted at two thirds between gonopore and spinnerets.

Body. Habitus as in Fig. 4. Ocular area slightly raised. Carapace with deep median pit and pair of very shallow indistinct furrows diverging from pit to posterior margin of carapace. Clypeus with very indistinct modified area medially (arrow in Fig. 129) and sclerotized margin. Sternum wider than long (1.05/0.70), unmodified. Abdomen oval to short cylindrical.

Chelicerae. As in Figs 119-120, with pair of frontal apophyses set with 2 and 3 modified hairs, respectively, with 23 modified hairs each on frontal face (Figs 130, 132); with strongly sculptured median margins (Fig. 131); with stridulatory ridges (Fig. 133).

PALPS. As in Figs 9-11; coxa with prominent retrolateral hump; trochanter barely modified; femur with distinct retrolateral process proximally, with stridulatory pick (modified hair) proximally on prolateral side, with transversal dark line retrolaterally, distally strongly widened and with rounded ventral protrusion; femur-patella joints shifted towards prolateral; tibia very large, thickest at two thirds, with two trichobothria; tibia-tarsus joints shifted towards retrolateral; procursus dorsally with $\sim 5$ weakly curved hairs, with strong hump on prolateral side. Procursus tip (Figs 113-114, 137-138) with scoop-shaped dorsal sclerite, strong hinged ventral sclerite (triangular in ventral view), and complex membranous central part with brush of hair-like processes on retrolateral side. Genital bulb (Figs 115-118) with basal

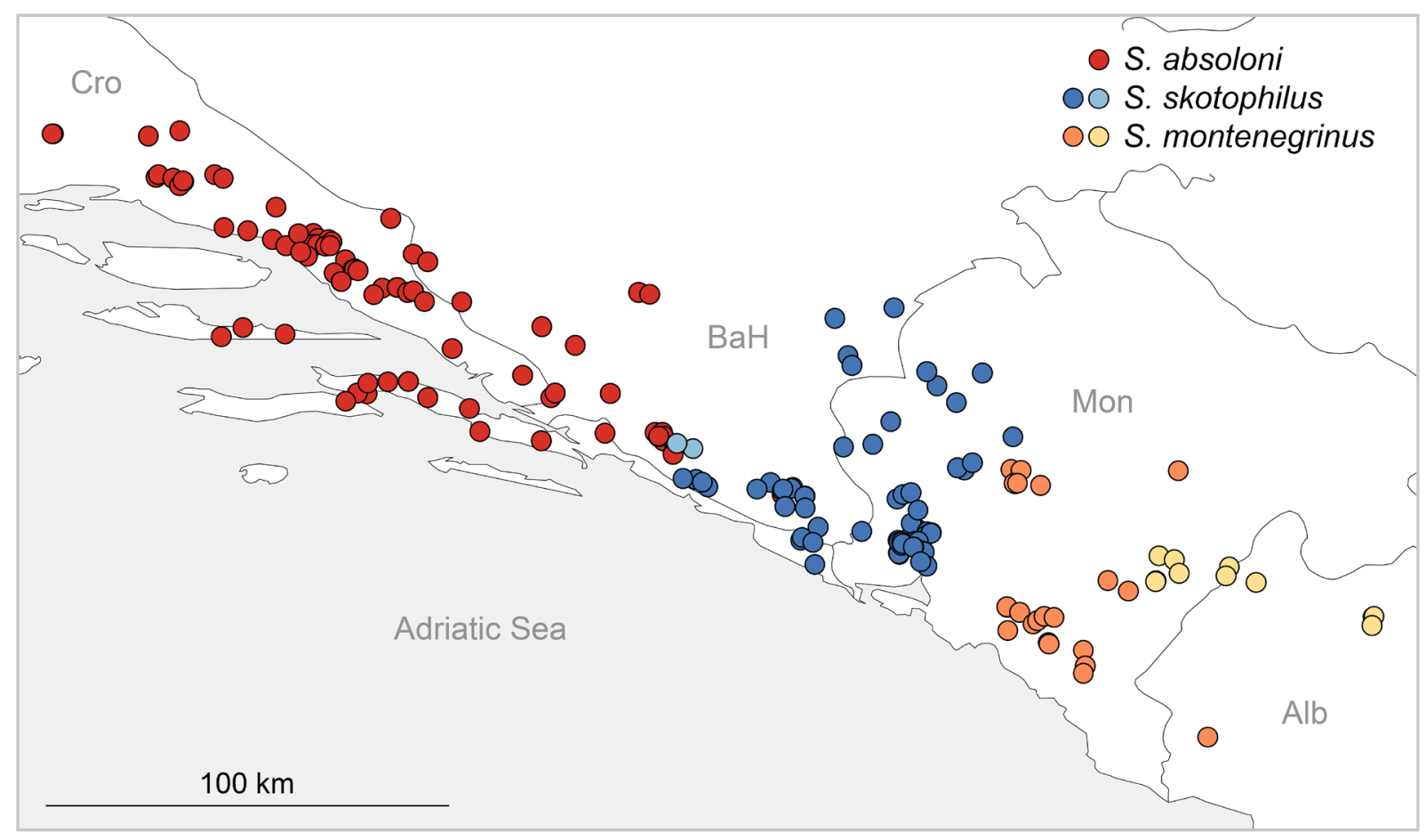

Fig. 168. Known distribution of the 'northern clade' of Stygopholcus Kratochvíl, 1932. Pale blue and pale orange marks denote specimens that are assigned tentatively to the respective species. Abbreviations: $\mathrm{Alb}=$ Albania; $\mathrm{BaH}=$ Bosnia and Herzegovina; $\mathrm{Cro}=$ Croatia; $\mathrm{Mon}=$ Montenegro. 
sclerite connecting to tarsus, small dorsal process weakly sclerotized, and main sclerite consisting of retrolateral process and dorsal process; retrolateral process distally widening, distal margin with pair of heavily sclerotized bulges on ventral side; dorsal process with single slightly curved tip, proximally with two small apophyses.

LEGs. With $\sim 12$ short spines in one row ventrally on femur 1; without curved hairs; few vertical hairs; retrolateral trichobothrium of tibia 1 at $2 \%$; prolateral trichobothrium absent on tibia 1, present on other tibiae; tarsus 1 with irregular platelets rather than with distinct pseudosegments.

Male (variation)

Color pattern consistent but individual elements slightly variable in size and distinctness; median section of ventral abdominal band sometimes divided into three parallel longitudinal bands; dark abdominal marks sometimes divided into many small round dots. Tibia $1(\mathrm{~N}=104)$ : 9.7-15.3 (mean 12.7); tibia 2 $(\mathrm{N}=41)$ : 6.5-10.4 (mean 8.4); spines on femur $1(\mathrm{~N}=11)$ : 0-34 (mean 19); modified hairs on cheliceral basis $(\mathrm{N}=50)$ : $19-44$ (mean 30); modified hairs on cheliceral apophysis $(\mathrm{N}=50)$ : 3-4 (mean 3.3); chelicerae maximum width $(\mathrm{N}=35)$ : $0.60-0.81$ (mean 0.70$)$; procursus length $(\mathrm{N}=35): 1.22-1.50$ (mean 1.36).

\section{Female}

In general similar to male (Fig. 5), but femur 1 without spines, clypeus unmodified. Without modifications posteriorly on carapace and anteriorly on abdomen. Tibia $1(\mathrm{~N}=91)$ : 8.9-13.7 (mean 11.2); tibia 2 $(\mathrm{N}=46)$ : 5.3-9.5 (mean 7.2); epigynum width $(\mathrm{N}=25)$ : 0.98-1.34 (mean 1.16); epigynum length $(\mathrm{N}=25)$ : $0.62-0.86$ (mean 0.72); epigynal measure $\mathrm{F}(\mathrm{N}=25)$ : $0.22-0.38$ (mean 0.29). Epigynum anterior plate roughly triangular, weakly protruding, with very strong transversal ridges in anterior part (Fig. 144); with internal structures visible in uncleared specimens: oval to drop-shaped anterior structure, short parallel (anteriorly slightly converging) sclerites posteriorly; posterior plate short but wide. Internal genitalia (Figs 121-122, 151-152) with distinctive ventral arc (Fig. 156; with several transversal, crescent-shaped sclerites and slightly elongate ventral pocket), with large pore plates (Fig. 122).

\section{Distribution}

Widely distributed in Greece (including Crete and the Ionian Islands) and southern Albania; the species seems to be largely absent from the Aegean Islands (except Thasos) (Fig. 167).

\section{Discussion}

\section{Stygopholcus montenegrinus - species or subspecies?}

Stygopholcus montenegrinus was originally described as a full species (Kratochvíl 1940) but later reduced to a subspecies of $S$. skotophilus by Senglet (1971). Senglet's argument focused on the small morphological differences between the two former species. While this observation is certainly correct, the conclusion is less evident. Countless species differ from the most similar species by minute differences. The most consistently applied criterion among arachnologists for separating species seems to be the presence of a gap, usually morphological, irrespective of how big the difference is. Kraus (2000) suggested that such gaps (usually in genital morphology) are almost universal in spiders, explaining the low number of subspecies in araneology.

Kraus (2000) certainly played down the problem of intraspecific genital variation ("There is practically no variation ... [in] ... the taxonomically decisive genital structures"), but it is a widespread pattern that genital structures are less variable within species than other morphological traits (Eberhard et al. 1998). As a result, there is less or no overlap among species, usually producing distinct gaps that facilitate 
taxonomic decisions. However, the majority of spider species are known from minimal samples, often from only one locality. Many of the observed gaps may thus be artifacts, resulting from insufficient knowledge. As a logical consequence, it has been argued that subspecies in general "cannot be known until the species is known" (Blackwelder 1967). Subspecies are arguably easier to justify in well-studied taxa like mammals, birds, and butterflies than in spiders.

In the case of Stygopholcus skotophilus and S. montenegrinus, our data, even though still limited, suggest that there are several small but consistent morphological (genital) differences and that there are no intermediate forms in the region where the two taxa come close to each other (they never share a locality). In addition, a multi-gene analysis of a limited sample of specimens resolved each taxon as monophyletic (L. Dietz et al., unpubl. data). This makes both taxa diagnosable, supposedly reproductively isolated, independent evolutionary lines, satisfying the main criteria of several major species concepts (Wheeler \& Meier 2000).

\section{Remarks on distribution}

The natural distribution of Smeringopinae (i.e., excluding synanthropic and anthropophilic species) ranges from South Africa to the Iberian Peninsula, the Balkan Peninsula, the Caucasus, and Central Asia (Huber 2011b). Stygopholcus thus marks the northern limit of Smeringopinae in Europe. In the absence of a reliable dated phylogeny of Stygopholcus and its putative closest relatives (Crossopriza, Holocnemus) (Eberle et al. 2018), the biogeographic history of Stygopholcus remains somewhat speculative. However, patterns paralleled in other groups give some indications about plausible scenarios.

First, the large majority of present-day Smeringopinae live in tropical and subtropical environments. Part of the present-day flora and fauna of the Balkan Peninsula is thought to constitute a 'PalaeoMediterranean' contingent that evolved under pre-Pliocene tropical climatic conditions (ScarasciaMugnozza et al. 2000). It is thus plausible that Stygopholcus is derived from ancestors that were present in the area before the Pliocene, in climatic conditions similar to those that its closest relatives still experience today.

Second, a relatively warm climate was maintained in the Balkan Peninsula during Pleistocene glaciations, particularly in the western part: glaciations in this area affected only higher elevations; orographic barriers prevented winter intrusions of cold polar air; and the Adriatic Sea had a moderating effect on temperatures along the coast (Mihevc et al. 2010). This made the Balkan Peninsula a refugium for many taxa that recolonized neighboring regions in the north after the last glaciation (Hewitt 2000). At the same time, however, glaciations resulted in drier climate (Sinopoli et al. 2019), and epigean ancestors of some present-day troglophile and troglobite taxa may have disappeared during this time. It is thus plausible that the epigean ancestor of the present-day northern clade of Stygopholcus disappeared during this period, and only descendants that had adapted to subterranean life survived. However, earlier climaterelated phenomena could have had the same effect, i.e., Stygopholcus may have adapted to subterranean conditions earlier than the Pleistocene. For example, a dry period at the end of the Miocene (Hsü et al. 1973) that is thought to have affected the salax group of the spider genus Troglohyphantes (DeelemanReinhold 1978); or the disappearance of Lake Pannon and the bordering Dinarid Lake System, which is thought to have caused the extinction of all epigean species of the bivalve genus Congeria (Bilandžija et al. 2013).

Third, taxa that exhibit a so-called 'mero-Dinaric' distribution may shed some light on the northern limit of Stygopholcus. The term mero-Dinaric was introduced for taxa that show either a northwest or southeast distribution in the Dinarides or a disjunct distribution, with the gap consistently in the same region in the central Dinarides (Sket 1994). Examples of mero-Dinaric taxa are species groups of the genus Troglohyphantes (Deeleman-Reinhold 1978), several genera of the spider family Dysderidae 
(Deeleman-Reinhold 1993; Pavlek \& Mammola 2021), and the diplopod subfamily Acherosomatinae (Antić et al. 2015). The northern limit of Stygopholcus matches closely to this gap, suggesting that the factors affecting mero-Dinaric taxa may also affect or have affected Stygopholcus. Reasons for this exclusive northwest or southeast distribution are largely unknown. One possible explanation is that in this area, much of the carbonate rocks that were exposed between $\sim 3-6$ Ma remained covered by impermeable flysch sediments for longer than in the neighboring areas, impeding access to the moistureretaining limestone habitat (Deeleman-Reinhold 1978; Sket 1994; Mihevc et al. 2010). This, together with decreasing temperatures towards the north may explain the northern limit of Stygopholcus, in spite of the ubiquity of suitable habitats (including caves) that would seem to allow for more northern occurrences and that have been sampled extensively.

Fourth, the Dinarides can be seen as ultimately continuing into Greece and the Greek islands, but a major distinction can be made in geological terms across the transition known as the Scutari-Peć transverse zone which runs through northern Albania, south of which the geology is dominated instead by volcanic or metamorphic rocks (Kissel et al. 1995, Reed et al. 2004). This geological line coincides closely with the current southern limit of the northern clade of Stygopholcus, suggesting that the presence of karst may have been a crucial factor in the evolution and survival of Stygopholcus in the western Balkan. However, Albania is relatively poorly sampled, so we cannot exclude the possibility that the southern limit of the northern clade is just a sampling artifact.

In summary, the northern clade of Stygopholcus may have been derived from an epigean ancestor that lived in the area before the Pliocene when the climate was more tropical or subtropical; during historical climatic perturbations the spiders survived because they could access exposed karst habitats in the southwest Dinarides; access to such habitats was presumably not possible further north and further south, for different reasons. The only surviving epigean congener, S. photophilus in Greece, may have largely retained the ancestral mode of life.

\section{Acknowledgments}

We thank the following people for sending material in loan: J. Altmann, C. Deltshev, P. Dolejš, J. Dunlop, A. Friederichs, J. Gruber, P. Jäger, J. Murphy, K. Paragamian, N. Platnick, C. Rollard, R. Salmaso, P. Schwendinger, L. Sorkin, V. Tomić, B. van der Bijl, and W. Wawer. Further specimens were kindly donated by F. Gasparo and B. Knoflach-Thaler. Many thanks to all members of the Croatian Biospeleological Society, to R. Ozimec, C. Deeleman-Reinhold, and all other speleologists and colleagues who collected the spider material. Thanks to the University Ljubljana for giving access to their collection. We are very thankful to D. Kurtović of the speleological society "Zelena Brda" in Trebinje for his help in clarifying and locating some caves in Bosnia \& Herzegovina during a field trip in 2014. The last author is thankful to G.S. Karaman for his invitation to the project "A catalogue of spiders (Arachnida, Araneae) of Montenegro", supported by the Montenegrin Academy of Sciences and Arts, and to V. Mijušković, S. Đuranović, M. Pavićević and A. Uskoković for their hospitality and support during his research in Montenegro. The first author is deeply indebted to R. Breitling and P. Dolejš for sharing their views on nomenclatorial issues; their insights have greatly improved the respective sections. For help with translation of Czech and Polish texts we thank P. Dolejš and W. Wawer. For help with georeferencing localities we thank C. Deltshev, K. Paragamian, and B. Vrenozi. We thank two anonymous reviewers for corrections and helpful suggestions.

\section{References}

Absolon K. \& Kratochvíl J. 1932. Zur Kenntnis der höhlenbewohnenden Araneae der illyrischen Karstgebiete. Mitteilungen über Höhlen- und Karstforschung 1932: 73-81. 
Antić D.Ž., Dražina T., Rađa T., Tomić V.T. \& Makarov S.E. 2015. Review of the family Anthogonidae (Diplopoda, Chordeumatida), with descriptions of three new species from the Balkan Peninsula. Zootaxa 3948: 151-181. https://doi.org/10.11646/zootaxa.3948.2.1

Bennett R. 2014. COSEWIC assessment and status report on the northwestern cellar spider Psilochorus hesperus in Canada. Committee on the Status of Endangered Wildlife in Canada, Ottawa. Available from www.registrelep-sararegistry.gc.ca/default_e.cfm [accessed 8 May 2021].

Bilandžija H., Morton B., Podnar M. \& Četković H. 2013. Evolutionary history of relict Congeria (Bivalvia: Dreissenidae): unearthing the subterranean biodiversity of the Dinaric Karst. Frontiers in Zoology 10: 5. Available from https://doi.org/10.1186/1742-9994-10-5 [accessed 8 May 2021].

Blackwelder R.E. 1967. Taxonomy. A Text and Reference Book. John Wiley, New York.

Bonnet P. 1958. Bibliographia araneorum. Analyse méthodique de toute la littérature aranéologique jusqu'en 1939. Tome II. Systématique des araignées (Étude par ordre alphabétique) (4me partie: N-S). Douladoure, Toulouse.

Bregović P., Fišer C. \& Zagmajster M. 2019. Contribution of rare and common species to subterranean species richness patterns. Ecology and Evolution 9: 11606-11618. https://doi.org/10.1002/ece3.5604

Brignoli P.M. 1971. Beitrag zur Kenntnis der mediterranen Pholcidae (Arachnida, Araneae). Mitteilungen aus dem Zoologischen Museum in Berlin 47: 255-267. https://doi.org/10.1002/mmnz.1971047020

Brignoli P.M. 1976. Ragni di Grecia IX. Specie nuove o interessanti delle famiglie Leptonetidae, Dysderidae, Pholcidae ed Agelenidae (Araneae). Revue suisse de Zoologie 83: 539578. https://doi.org/10.5962/bhl.part.91452

Brignoli P.M. 1983. A Catalogue of the Araneae described between 1940 and 1981. Manchester University Press, Manchester.

Brown B.V. 1993. A further chemical alternative to critical-point-drying for preparing small (or large) flies. Fly Times 11: 10.

Deeleman-Reinhold C.L. 1978. Revision of the Cave-dwelling and related Spiders of the Genus Troglohyphantes Joseph (Linyphiidae), with special Reference to the Yugoslav Species. Academia Scientiarum et Artium Slovenica, Ljubljana.

Deeleman-Reinhold C.L. 1993. The genus Rhode and the harpacteine genera Stalagtia, Folkia, Minotauria, and Kaemis (Araneae, Dysderidae) of Yugoslavia and Crete, with remarks on the genus Harpactea. Revue arachnologique 10: 105-135.

Deeleman-Reinhold C.L. \& Deeleman P.R. 1988. Revision des Dysderinae (Araneae, Dysderidae), les espèces mediterranéennes occidentales exceptées. Tijdschrift voor Entomologie 131: 141-269.

Deltshev C.D. 1985. New data concerning cave spiders (Araneae) in Greece with description of a new Leptonetela (Araneae, Leptonetidae). Acta zoologica Bulgarica 27: 41-45.

Deltshev C.D. 2004. A zoogeographical review of the spiders (Araneae) of the Balkan Peninsula. In: Griffiths H.I., Kryštufek B. \& Reed J.M. (eds) Balkan Biodiversity. Pattern and Process in the European Hotspot: 193-200. Springer, Dordrecht. https://doi.org/10.1007/978-1-4020-2854-0

Deltshev C.D., Vrenosi B., Blagoev G. \& Lazarov S. 2011. Spiders of Albania - faunistic and zoogeographical review (Arachnida: Araneae). Acta zoologica Bulgarica 63: 125-144.

Džukić G. \& Kalezić M.L. 2004. The biodiversity of amphibians and reptiles in the Balkan Peninsula. In: Griffiths H.I., Kryštufek B. \& Reed J.M. (eds) Balkan Biodiversity. Pattern and Process in the European Hotspot: 167-192. Springer, Dordrecht. https://doi.org/10.1007/978-1-4020-2854-0 
Eastwood W.J. 2004. East Mediterranean vegetation and climate change. In: Griffiths H.I., Kryštufek B. \& Reed J.M. (eds) Balkan Biodiversity. Pattern and Process in the European Hotspot: 25-48. Springer, Dordrecht. https://doi.org/10.1007/978-1-4020-2854-0

Eberhard W.G., Huber B.A., Rodriguez R.L., Briceño D., Salas I. \& Rodriguez V. 1998. One size fits all? Relationships between the size of genitalia and other body parts in 20 species of insects and spiders. Evolution 52: 415-431. https://doi.org/10.2307/2411078

Eberle J., Dimitrov D., Valdez-Mondragón A. \& Huber B.A. 2018. Microhabitat change drives diversification in pholcid spiders. BMC Evolutionary Biology 18: 141. https://doi.org/10.1186/s12862-018-1244-8

Gottstein Matočec S., Bakran-Petricioli T., Bedek J., Bukovec D., Buzjak S., Franičević M., Jalžić B., Kerovec M., Kletečki E., Kralj J., Kružić P., Kučinić M., Kuhta M., Matočec N., Ozimec R., Rađa T., Štamol V., Ternjej I. \& Tvrtković N. 2002. An overview of the cave and interstitial biota of Croatia. Natura Croatica 11: 1-112.

Griffiths H.I., Kryštufek B. \& Reed J.M. 2004. Balkan Biodiversity. Pattern and Process in the European Hotspot. Springer, Dordrecht. https://doi.org/10.1007/978-1-4020-2854-0

Hewitt G. 2000. The genetic legacy of the Quaternary ice ages. Nature 405: 907-913. https://doi.org/10.1038/35016000

Hsü K., Ryan W.B.F. \& Cita M. 1973. Late Miocene desiccation of the Mediterranean. Nature 242: 240-244. https://doi.org/10.1038/242240a0

Huber B.A. 2000. New World pholcid spiders (Araneae: Pholcidae): a revision at generic level. Bulletin of the American Museum of Natural History 254: 1-348. https://doi.org/10.1206/0003-0090(2000)254<0001:NWPSAP >2.0.CO;2

Huber B.A. 2009. Life on leaves: leaf-dwelling pholcids of Guinea, with emphasis on Crossopriza cylindrogaster, a spider with inverted resting position, pseudo-eyes, lampshade web, and tetrahedral egg-sac (Araneae: Pholcidae). Journal of Natural History 43: 2491-2523. https://doi.org/10.1080/00222930903207876

Huber B.A. 2011a. Revision and cladistic analysis of Pholcus and closely related taxa (Araneae, Pholcidae). Bonner zoologische Monographien 58: 1-509.

Huber B.A. 2011b. Phylogeny and classification of Pholcidae (Araneae): an update. The Journal of Arachnology 39: 211-222. https://doi.org/10.1636/CA10-57.1

Huber B.A. 2012. Revision and cladistic analysis of the Afrotropical endemic genus Smeringopus Simon, 1890 (Araneae: Pholcidae). Zootaxa 3461: 1-138.

Huber B.A. 2013. Revision and cladistic analysis of the Guineo-Congolian spider genus Smeringopina Kraus (Araneae, Pholcidae). Zootaxa 3713: 1-160. https://d.doi.org/10.11646/zootaxa.3713.1.1

Huber B.A. 2014. Pholcidae. In: Roig-Juñent S., Claps L.E. \& Morrone J.J. (eds) Biodiversidad de Artrópodos Argentinos, Vol. 3: 131-140. Sociedad Entomológica Argentina.

Huber B.A. 2020. Revision of the spider genus Hoplopholcus Kulczyński (Araneae, Pholcidae). Zootaxa 4726: 1-94. https://doi.org/10.11646/zootaxa.4726.1.1

Huber B.A. \& Chao A. 2019. Inferring global species richness from megatransect data and undetected species estimates. Contributions to Zoology 88: 42-53. https://doi.org/10.1163/18759866-20191347

Huber B.A. \& Fleckenstein N. 2008. Comb-hairs on the fourth tarsi in pholcid spiders (Araneae, Pholcidae). Journal of Arachnology 36: 232-240. https://doi.org/10.1636/CSh07-71.1 
ICZN. 1999. International Code of Zoological Nomenclature. Fourth edition. London, U.K. International Trust for Zoological Nomenclature. Available from https://www.iczn.org/the-code/ [accessed 8 May 2021].

Kissel C., Speranza F. \& Milicevic V. 1995. Paleomagnetism of external southern and central Dinarides and northern Albanides: Implications for the Cenozoic activity of the Scutari-Pec transverse zone. Journal of Geophysical Research 100: 14999-15007. https://doi.org/10.1029/95JB01243

Komnenov M. 2009. Checklist of spiders (Araneae) of Bosnia and Herzegovina. Prilozi fauni Bosne $i$ Hercegovine 5: 51-69.

Komnenov M. 2020. Subterranean spiders (Arachnida, Araneae) of Montenegro. The Montenegrin Academy of Sciences and Arts, Proceedings of the Section of Natural Sciences 23 (2019): 119-169.

Kratochvíl J. 1932. Přispěvek k poznání Araneid střední Slavonie. Sborník Vysoké Školy Zemědělské, Brno (C) 23: 1-16.

Kratochvíl J. 1934. Liste générale des Araignées cavernicoles en Yugoslavie. Pregled pecinskih paukova u Jugoslaviji. Prirodoslovne Razprave 2: 165-226.

Kratochvíl J. 1935. Araignées cavernicoles de Krivošije. Práce Moravské Přirodovědecké Společnosti 9: $1-25$.

Kratochvíl J. 1940. Etude sur les araignées cavernicoles du genre Stygopholcus Krat. Acta Societatis Scientiarum Naturalium Moraviae 12: 1-26.

Kratochvíl J. 1978. Araignées cavernicoles des îles Dalmates. Přrirodovědné práce ústavů Československé Akademie Věd v Brně (N.S.) 12: 1-59.

Kraus O. 2000. Why no subspecies in spiders? In: Toft S. \& Scharff N. (eds) European Arachnology 2000: Proceedings of the 19th European Colloquium of Arachnology, Arhus: 303-314. Aarhus University Press, Aarhus.

Kryštufek B. \& Reed J.M. 2004. Pattern and process in Balkan biodiversity - an overview. In: Griffiths H.I., Kryštufek B. \& Reed J.M. (eds) Balkan Biodiversity. Pattern and Process in the European Hotspot: 1-8. Springer, Dordrecht. https://doi.org/10.1007/978-1-4020-2854-0

Kulczyński W. 1914. Aranearum species novae minusve cognitae, in montibus Kras dictis a Dre C. Absolon aliisque collectae. Bulletin international de l'Academie des Sciences de Cracovie 1914: 353387, pl. 16.

Lukić M., Delić T., Pavlek M., Deharveng L. \& Zagmajster M. 2020. Distribution pattern and radiation of the European subterranean genus Verhoeffiella (Collembola, Entomobryidae). Zoologica Scripta 49: 86-100. https://doi.org/10.1111/zsc. 12392

Mammola S., Cardoso P., Angyal D., Balázs G., Blick T., Brustel H., Carter J., Ćurčić S., Danflous S., Dányi L., Déjean S., Deltshev C., Elverici M., Fernández F., Gasparo F., Komnenov M., Komposch C., Kováč L., Kunt K.B., Mock A., Moldovan O.T., Naumova M., Pavlek M., Prieto C.E., Ribera C., Rozwałka R., Růžička V., Vargovitsh R.S., Zaenker S. \& Isaia M. 2019. Local- versus broad-scale environmental drivers of continental $\beta$-diversity patterns in subterranean spider communities across Europe. Proceedings of the Royal Society B: Biological Sciences 286: 1-9. https://doi.org/10.1098/rspb.2019.1579

Marusik Y.M. \& Koponen S. 2002. Diversity of spiders in boreal and arctic zones. The Journal of Arachnology 30: 205-210. https://doi.org/10.1636/0161-8202(2002)030[0205:DOSIBA]2.0.CO;2

Mihevc A., Prelovšek M. \& Zupan Hajna N. 2010. Introduction to the Dinaric Karst. Karst Research Institute and Research Centre of the Slovenian Academy of Sciences and Arts, Postojna, Slovenia. 
Naumova M., Lazarov S.P., Petrov B.P. \& Deltshev C.D. 2016. New faunistic data on the cave-dwelling spiders in the Balkan Peninsula (Araneae). Ecologica Montenegrina 7: 425-438.

Naumova M., Lazarov S. \& Deltshev C.D. 2019. Faunistic diversity of the spiders in Montenegro (Arachnida: Araneae). Ecologica Montenegrina 22: 50-89. https://doi.org/10.37828/em.2019.22.5

Nentwig W., Blick T., Bosmans R., Gloor D., Hänggi A. \& Kropf C. 2020. Spinnen Europas. Version November 2020. Available from https://www.araneae.nmbe.ch [accessed 5 Nov. 2020]. https://doi.org/10.24436/1

Pavlek M. \& Mammola S. 2021. Niche-based processes explaining the distributions of closely related subterranean spiders. Journal of Biogeography 48: 118-133. https://doi.org/10.1111/jbi.13987

Platania L., Pavlek M. \& Arnedo M. 2020. Testing the monophyly of the ground-dweller spider genus Harpactea Bristowe, 1939 (Araneae, Dysderidae) with the description of three new species. Systematics and Biodiversity 18: 688-707. https://doi.org/10.1080/14772000.2020.1776786

Platnick N.I. 1993. Advances in Spider Taxonomy 1988-1991, with Synonymies and Transfers 19401980. The New York Entomological Society, New York.

Platnick N.I. 2000. The World Spider Catalog, Version 1.0. Available from https://wsc.nmbe.ch/archive/ [accessed 8 May 2021].

Platnick N.I. 2002. The World Spider Catalog, Version 2.5. Available from https://wsc.nmbe.ch/archive/ [accessed 8 May 2021].

Reed J.M., Kryštufek B. \& Eastwood W.J. 2004. The physical geography of the Balkans and nomenclature of place names. In: Griffiths H.I., Kryštufek B. \& Reed J.M. (eds) Balkan Biodiversity. Pattern and Process in the European Hotspot: 9-22. Springer, Dordrecht. https://doi.org/10.1007/978-1-4020-2854-0

Roewer C.F. 1942. Katalog der Araneae von 1758 bis 1940. 1. Band (Mesothelae, Orthognatha, Labidognatha: Dysderaeformia, Scytodiformia, Pholciformia, Zodariiformia, Hersiliaeformia, Argyopiformia). Natura, Buchhandlung für Naturkunde und exakte Wissenschaften Paul Budy, Bremen.

Růžička V., Kůrka A., Buchar J. \& Řezáč M. 2005. Czech Republic - the type material of spiders (Araneae). Časopis Národního muzea, ̌̌ada přirodovédná 174: 13-64.

Scarascia-Mugnozza G., Oswald H., Piussi P. \& Radoglou K. 2000. Forests in the Mediterranean region: gaps in knowledge and research needs. Forest Ecology and Management 132: 97-109. https://doi.org/10.1016/S0378-1127(00)00383-2

Senglet A. 1971. Note sur les Pholcidae (Arachn.) de Grèce. Mitteilungen der Schweizerischen Entomologischen Gesellschaft 44: 345-359. https://doi.org/10.5169/seals-401666

Senglet A. 2001. Copulatory mechanisms in Hoplopholcus, Stygopholcus (revalidated), Pholcus, Spermophora and Spermophorides (Araneae, Pholcidae), with additional faunistic and taxonomic data. Mitteilungen der Schweizerischen Entomologischen Gesellschaft 74: 43-67. https://doi.org/10.5169/seals-402796

Sinopoli G., Peyron O., Masi A., Holtvoeth J., Francke A., Wagner B. \& Sadori L. 2019. Pollen-based temperature and precipitation changes in the Ohrid Basin (western Balkans) between 160 and $70 \mathrm{ka}$. Climate of the Past 15: 53-71. https://doi.org/10.5194/cp-15-53-2019

Sket B. 1994. Distribution patterns of some subterranean Crustacea in the territory of the former Yugoslavia. Hydrobiologia 287: 65-75. https://doi.org/10.1007/BF00006897

Sket B., Paragamian K. \& Trontelj P. 2004. A census of the obligate subterranean fauna of the Balkan Peninsula. In: Griffiths H.I., Kryštufek B. \& Reed J.M. (eds) Balkan Biodiversity. Pattern and Process in the European Hotspot: 49-68. Springer, Dordrecht. https://doi.org/10.1007/978-1-4020-2854-0 
Tomić V.T., Ćurčić S.B. \& Pešić V.M. 2000. Contribution to the study of some cavernicolous spiders (Arachnida, Araneae) in Montenegro. Archives of Biological Sciences, Belgrade 52: 35P-36P.

Tzedakis P.C. 2004. The Balkans as prime glacial refugial territory of European temperate trees. In: Griffiths H.I., Kryštufek B. \& Reed J.M. (eds) Balkan Biodiversity. Pattern and Process in the European Hotspot: 49-68. Springer, Dordrecht. https://doi.org/10.1007/978-1-4020-2854-0

Wheeler Q.D. \& Meier R. 2000. Species Concepts and Phylogenetic Theory. A Debate. Columbia University Press, New York.

World Spider Catalog. 2020. World Spider Catalog. Version 21.5. Natural History Museum Bern. Available from http://wsc.nmbe.ch [accessed 5 Nov. 2020]. https://doi.org/10.24436/2

Manuscript received: 18 January 2021

Manuscript accepted: 15 March 2021

Published on: 11 June 2021

Topic editor: Rudy Jocqué

Section editor: Maria Chatzaki

Desk editor: Eva-Maria Levermann

Printed versions of all papers are also deposited in the libraries of the institutes that are members of the EJT consortium: Muséum national d'histoire naturelle, Paris, France; Meise Botanic Garden, Belgium; Royal Museum for Central Africa, Tervuren, Belgium; Royal Belgian Institute of Natural Sciences, Brussels, Belgium; Natural History Museum of Denmark, Copenhagen, Denmark; Naturalis Biodiversity Center, Leiden, the Netherlands; Museo Nacional de Ciencias Naturales-CSIC, Madrid, Spain; Real Jardín Botánico de Madrid CSIC, Spain; Zoological Research Museum Alexander Koenig, Bonn, Germany; National Museum, Prague, Czech Republic. 